\title{
The Parity Operator:
}

\section{applications in quantum metrology}

Richard J. Birrittella, ${ }^{1, a)}$ Paul M. Alsing, ${ }^{1, b)}$ and Christopher C. Gerry, ${ }^{2,(c)}$

1) Air Force Research Laboratory, Information Directorate, Rome, NY, USA, 13441

${ }^{2)}$ Department of Physics and Astronomy, Lehman College, The City University of New York, Bronx, New York, 10468-1589, USA

(Dated: 21 August 2020)

In this paper, we review the use of parity as a detection observable in quantum metrology as well as introduce some original findings with regards to measurement resolution in Ramsey spectroscopy and quantum non-demolition (QND) measures of atomic parity. Parity was first introduced in the context of Ramsey spectroscopy as an alternative to atomic state detection. It was latter adapted for use in quantum optical interferometry where it has been shown to be the optimal detection observable saturating the quantum Cramér-Rao bound for path symmetric states. We include a brief review of the basics of phase estimation and the connection between parity-based detection and the quantum Fisher information as it applies to quantum optical interferometry. We also discuss the efforts made in experimental methods of measuring photon-number parity and close the paper with a discussion on the use of parity leading to enhanced measurement resolution in multi-atom spectroscopy. We show how this may be of use in the construction of high-precision multiatom atomic clocks.

We dedicate this paper to the memory of Jonathan $P$. Dowling, whose body of work had a tremendous impact on the fields of quantum optical interferometry and quantum metrology.

\section{CONTENTS}

\section{Introduction}

II. Ramsey spectroscopy with entangled and unentangled atoms

III. Phase estimation and the quantum Cramér-Rao bound

A. The Cramér-Rao lower bound

B. Quantum Fisher information and the upper bound

1. Calculating the QFI for pure and mixed states

2. Connection to parity-based detection

C. Calculating the QFI in quantum optical interferometry

IV. Quantum Optical Interferometry
A. The N00N states
B. Entangled coherent states
C. Twin-Fock state input 1. Superpositions of twin-Fock states
D. Coherent light mixed with a squeezed vacuum state
E. Coherent light mixed with $N$ photons

\footnotetext{
a)Electronic mail: richard.birrittella@ lehman.cuny.edu

b) Electronic mail: paul.alsing@us.af.mil.

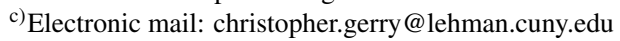

1. Measurement resolution using parity-based detection

2. Phase uncertainty: approaching the Heisenberg limit

F. Interferometry with strictly classical (coherent) light

1. Difference in output mode intensities

2. Parity-based detection

\section{Experimental realizations of parity-based} detection

A. QND measures of atomic parity

1. Coupling to an ancillary atomic system 20

2. Coupling to a field state

B. Detection of photon parity

C. Quantum random number generator

VI. Enhanced measurement resolution in multi-atom atomic clocks

A. Population difference for an arbitrary atomic state

B. Parity-based resolution for an arbitrary atomic state

1. Unentangled ensemble of atoms 26

2. Entangled atomic ensemble

VII. Conclusion

Acknowledgments

A. A brief review of the $\mathrm{SU}(2)$ group

1. The Dicke states

a. The atomic coherent state

2. The Schwinger realization of SU(2)

3. Beam splitter transformations

B. The Wigner- $d$ matrix elements

1. Defined

2. Useful identities 


\section{INTRODUCTION}

Observables in quantum mechanics, which are generally based on their classical counterparts, are represented as Hermitian operators in Hilbert space due to the fact that such operators possess real eigenvalues. According to orthodox quantum mechanics, the measurement of an observable returns a corresponding eigenvalue of the operator with probabilities of obtaining specific eigenvalues determined by the details of the prepared state vector. Usual quantum observables most commonly discussed are energy, position, momentum, angular momentum, etc., all of which have classical analogs. The spin degree of freedom of an electron is often taken as a quantum observable with no classical analog which, of course, is true in the sense that it is not possible to make sense classically out of the notion of a point particle having spin angular momentum. Yet spin angular momentum itself surely exists in the classical world of macroscopic objects.

The same cannot be said, however, for the concept of photon-number parity. Let $|n\rangle$, where $n \in \mathbb{Z}^{+}$, be a Fock state for a single-mode quantized electromagnetic field. The photon-number parity of the state is defined as the evenness or oddness of the number as quantified by $(-1)^{n}$. We define the usual boson operators $\hat{a}$ and $\hat{a}^{\dagger}$, the annihilation and creation operators of the field, respectively, which satisfy the usual commutation relation $\left[\hat{a}, \hat{a}^{\dagger}\right]=1$ and the number operator $\hat{n}=\hat{a}^{\dagger} \hat{a}$ such that $\hat{n}|n\rangle=n|n\rangle$. In terms of these operators, one can introduce the photon number parity operator $\hat{\Pi}=(-1)^{\hat{a}^{\dagger} \hat{a}}=e^{i \pi \hat{a}^{\dagger} \hat{a}}$ such that $\hat{\Pi}|n\rangle=(-1)^{n}|n\rangle$. The eigenvalues of this operator are dichotomic and thus highly degenerate. While it is clear that $\hat{\Pi}$ is Hermitian and thus constitutes an observable, it can also be shown that there exists no classical analog to photon number parity. This can be demonstrated by considering the energies of the quantized field: while these energies are discrete (i.e. $E_{n}=\hbar \omega\left(n+\frac{1}{2}\right)$ ), the energies of a classical field are continuous.

The parity operator makes frequent appearances in quantum optics and quantum mechanics. For example it has been pointed out that the quasi-probability distribution known as the Wigner function $1[2]$ can be expressed in terms of the expectation value of the displaced parity operator ${ }^{3}$ given for a single-mode field as

$$
W(\alpha)=\frac{2}{\pi}\left\langle\hat{D}(\alpha) \hat{\Pi} \hat{D}^{\dagger}(\alpha)\right\rangle
$$

where $\hat{D}(\alpha)=e^{\alpha \hat{a}^{\dagger}-\alpha^{*} \hat{a}}$ is the usual displacement operator familiar from quantum optics, and where $\alpha$ is the displacement amplitude generally taken to be a complex number. This relationship is the basis for reconstructing a field state through quantum state tomography 4 . The parity operator has also appeared in various proposals for testing highly excited entangled two-mode field states for violations of a Bell's inequality 5 .
Another field in which parity sees use is quantum metrology, where it serves as a suitable detection observable for reasons we will endeavor to address. Quantum metrology is the science of using quantum mechanical states of light or matter in order to perform highly resolved and sensitive measurements of weak signals like those expected by gravitational wave detectors (see for example Barsotti et al. ${ }^{6}$ and references therein) and for the precise measurements of transition frequencies in atomic or ion spectroscopy ${ }^{7}$. This is often done by exploiting an inherently quantum property of the state such as entanglement and/or squeezing. The goal of quantum metrology is to obtain greater sensitivities in the measurement of phase-shifts beyond what is possible with classical resources alone, which at best can yield sensitivities at the shot noise-, or standard quantum limit (SQL). For an interferometer operating with classical (laser) light, the sensitivity of a phase-shift measurement, $\Delta \varphi$, scales as $1 / \sqrt{\bar{n}}$ where $\bar{n}$ is the average photon number of the laser field. This defines the SQL as the greatest sensitivity obtainable using classical light: $\Delta \varphi_{\mathrm{SQL}}=1 / \sqrt{\bar{n}}$. In cases where phase-shifts are due to linear interactions, the optimal sensitivity allowed by quantum mechanics is known as the Heisenberg limit (HL), defined as $\Delta \varphi_{\mathrm{HL}}=1 / \bar{n}$. Only certain states of light having no classical analog, including entangled states of light, are capable of breaching the SQL level of sensitivity. In many cases, perhaps even most, reaching the HL requires not only a highly non-classical state of light but also a special observable to be measured. That observable turns out to be photon-number parity measured at one of the output ports of the interferometer. This is because the usual technique of subtracting irradiances of the two output beams of the interferometers fails for many important non-classical states. Furthermore, as to be discussed in latter sections, consideration of the quantum Fisher information indicates that photon number parity serves as the optimal detection observable for path symmetric states in quantum optical interferometry.

This paper is organized as follows: Section [I] begins with a discussion on the origin of parity-based measurement in the context of atomic spectroscopy. In section III we present a brief review of the basics of phase estimation including a concise derivation of the quantum Cramér-Rao bound and the related quantum Fisher information and discuss how these relate to parity-based measurement, particularly in the field of quantum optical interferometry. In Section IV we highlight the use of several relevant states of light in quantum optical interferometry such as the NOON states and coherent light. We show in the latter case that the use of parity does not yield sensitivity (i.e. reduced phase uncertainty) beyond the classical limit but does enhance measurement resolution. In Section $\nabla$ we discuss the experimental efforts made in performing photonic parity measurements. Finally, in Section VI we briefly return to the atomic population parity measurements, this time in the context of atomic coherent states and show that such measurements could lead to high-resolution multi-atom atomic clocks, i.e. atomic clocks of greater precision than is currently available. We conclude the paper with some brief remarks. 


\section{RAMSEY SPECTROSCOPY WITH ENTANGLED AND UNENTANGLED ATOMS}

We first introduce the Dicke atomic pseudo-angular momentum operators for a collection of $N$ two-level atoms. These are given $b y$

$$
\hat{J}_{x, y, z}=\frac{1}{2} \sum_{i=1}^{N} \sigma_{x, y, z}^{(i)}
$$

satisfying the su(2) commutation relation of Eq. A13 (see Appendix A 2) and where $\sigma_{x, y, z}^{(i)}$ are the Pauli operators for the $i^{\text {th }}$ atom as given by $\sigma_{x}^{(i)}=|e\rangle_{i}\langle g|+| g\rangle_{i}\langle e|, \sigma_{y}^{(i)}=$ $-i\left(|e\rangle_{i}\langle g|-| g\rangle_{i}\langle e|\right), \sigma_{z}^{(i)}=|e\rangle_{i}\langle e|-| g\rangle_{i}\langle g|$ and where the ground and excited states for the $i^{\text {th }}$ atom are denoted $|g\rangle_{i},|e\rangle_{i}$, respectively. Obviously the operators given in Eq. 2 are additive over all atoms. We now introduce the corresponding the collective atomic states, the Dicke states, expressed in terms of the $\mathrm{SU}(2)$ angular momentum states $|j, m\rangle$ where $j=$ $N / 2$ and $m \in\{-j,-j+1, \ldots, j\}$ which can be given as superpositions of the product states of all atoms. For $N=2 j$ atoms, with $j=1 / 2,1, \ldots$, the Dicke states $|j, m\rangle$ are defined in terms of the individual atomic states as $|j, j\rangle=\left|e_{1}, e_{2}, \ldots e_{N}\right\rangle=|e\rangle^{\otimes N}$ and $|j,-j\rangle=\left|g_{1}, g_{2}, \ldots g_{N}\right\rangle=|g\rangle^{\otimes N}$ with intermediate steps consisting of superpositions of all permutations with consecutively more atoms being found in the ground state all the way down to $|j,-j\rangle$, where all atoms are in the ground state. The ladder operators given as

$$
\begin{aligned}
& \hat{J_{+}}=\hat{J_{x}}+i \hat{J_{y}}=\sum_{i=1}^{N}|e\rangle_{i}\langle g| \\
& \hat{J_{-}}=\hat{J_{x}}-i \hat{J_{y}}=\sum_{i=1}^{N}|g\rangle_{i}\langle e|
\end{aligned}
$$

can be used repeatedly to generate expressions for all the non-extremal states in terms of the individual atoms. We relegate further discussion of the mapping between the two sets of states to Appendix A

In what follows we denote the exact transition frequency between the excited and ground states as $\omega_{0}$. The goal of Ramsey spectroscopy is to determine the frequency with as high a sensitivity (or as low an uncertainty) as possible. We first go through the Ramsey procedure with a single atom assumed initially in the ground state such that $\mid$ in $\rangle=|g\rangle$. The atom is subjected to a $\pi / 2$ pulse described by the operator $e^{-i \frac{\pi}{2} \hat{J}_{y}}$ and implemented with radiation of frequency $\omega$. For one atom, the rotation operator about the $y$-axis for an arbitrary angle is given by

$$
e^{-i \beta \hat{J_{y}}}=e^{-i \frac{\beta}{2} \sigma_{y}}=\boldsymbol{I}_{2} \cos (\beta / 2)-i \sigma_{y} \sin (\beta / 2),
$$

where $\boldsymbol{I}_{2}=|e\rangle\langle e|+| g\rangle\langle g|$ is the identity operator in this twodimensional subspace. This results in the transformations

$$
e^{-i \beta \hat{J}_{y}}\left[\begin{array}{l}
|e\rangle \\
|g\rangle
\end{array}\right]=\left[\begin{array}{l}
\cos (\beta / 2)|e\rangle+\sin (\beta / 2)|g\rangle \\
\cos (\beta / 2)|g\rangle-\sin (\beta / 2)|e\rangle
\end{array}\right],
$$

which for $\beta=\pi / 2$ we have the balanced superpositions

$$
\begin{aligned}
& e^{-i \frac{\pi}{2} \hat{J}_{y}}|e\rangle=\frac{1}{\sqrt{2}}(|g\rangle+|e\rangle), \\
& e^{-i \frac{\pi}{2} \hat{J}_{y}}|g\rangle=\frac{1}{\sqrt{2}}(|g\rangle-|e\rangle) .
\end{aligned}
$$

Assuming the atom is initially in the ground state, the state of the atom after the first $\pi / 2$ pulse becomes $\left|\psi\left(t_{\pi / 2}\right)\right\rangle=$ $e^{-i \frac{\pi}{2} \hat{J}_{y}} \mid$ in $\rangle=\frac{1}{\sqrt{2}}(|g\rangle-|e\rangle)$. This is followed by a period $T$ of free evolution (precession) governed by the operator $e^{-i\left(\omega_{0}-\omega\right) T \hat{J}_{z}}$ where once again $\omega$ is the frequency of the radiation field implementing $\pi / 2$ pulses. The state after free evolution is

$$
\left|\psi\left(t_{\pi / 2}+T\right)\right\rangle=\frac{1}{\sqrt{2}}\left(e^{i \frac{\phi}{2}}|g\rangle-e^{-i \frac{\phi}{2}}|e\rangle\right),
$$

where we have set $\phi=\left(\omega_{0}-\omega\right) T$. After the second $\pi / 2$ pulse following free evolution, we use Eq. 6 to find the final state

$$
\left|\psi\left(t_{f}\right)\right\rangle=i \sin (\phi / 2)|g\rangle-\cos (\phi / 2)|e\rangle
$$

where $t_{f}=2 t_{\pi / 2}+T$ and where it is assumed $T \gg 2 t_{\pi / 2}$ such that $t_{f} \simeq T$. A diagram of the transformations in Bloch-sphere representation is provided in Fig. 1 as well as an idealized sketch of the Ramsey technique in Fig. 2. The expectation value of $\hat{J}_{z}$ for this state is

$$
\left\langle\hat{J}_{z}(\phi)\right\rangle_{t_{f}}^{(1)}=\frac{1}{2} \cos \phi=\frac{1}{2} \cos \left[\left(\omega_{0}-\omega\right) T\right] .
$$

By tuning the frequency of the driving field so as to maximize $\left\langle\hat{J}_{z}(\phi)\right\rangle_{t_{f}}^{(1)}$, one can estimate the transition frequency $\omega_{0}$.

If we consider $N$ atoms one at a time or collectively through the Ramsey procedure ${ }^{9}$, then the initial state with all atoms in their ground state is the Dicke state $|j,-j\rangle$, and after the first $\pi / 2$ pulse, the state generated $|\psi\rangle=e^{-i \frac{\pi}{2} \hat{J}_{y}}|j,-j\rangle$ is an example of an atomic coherent state (ACS) $\sqrt{10}$. For a brief review of the Dicke states as well as a derivation of the ACS, see Appendix A 1. In such a state there is no entanglement among the atoms: each atom undergoes the same evolution through the Ramsey process and, because the $\hat{J}_{z}$ operator is the sum $\hat{J_{z}}=\frac{1}{2} \sum_{i}^{N} \sigma^{(i)_{z}}$, one easily finds that 


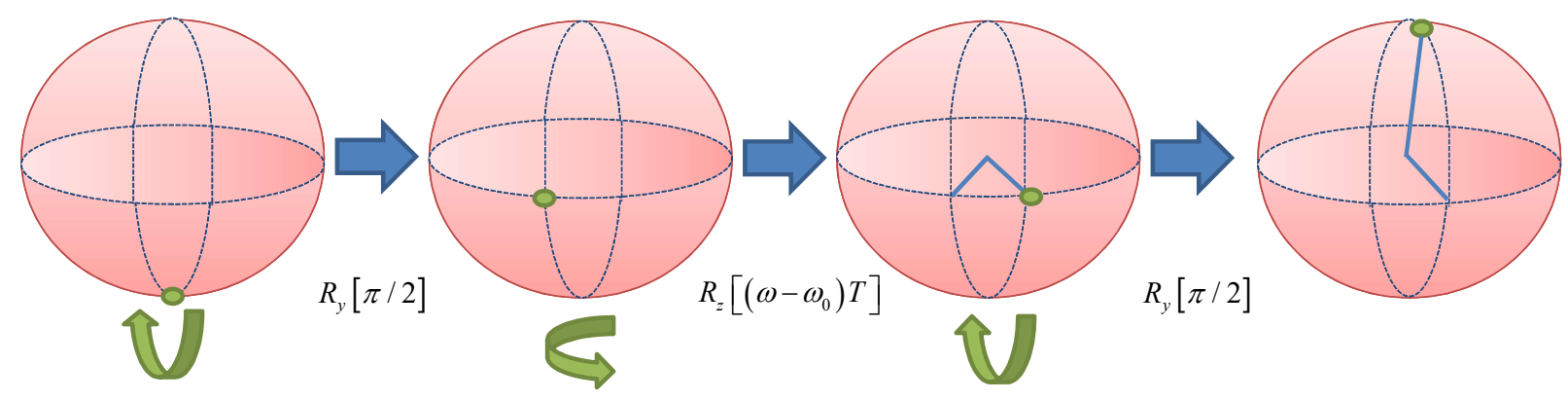

FIG. 1. Bloch sphere representation for single-atom spectroscopy. The atom is intially in its ground state. The first $\pi / 2$-pulse (at frequency $\omega)$ places the atom in a superposition state. The free evolution of time $T$ corresponds to a rotation in the $x-y$ plane. The final $\pi / 2$-pulse (also at frequency $\omega$ ) places the state of the atom near the excited state. For a frequency-spread in radiation $\Delta \omega$ the state will be nearly at the north pole of the Bloch sphere. Note $R_{i}[\theta]$ represents rotation by $\theta$ about the $i$-axis.

$$
\left\langle\hat{J}_{z}(\phi)\right\rangle_{t_{f}}^{(\mathrm{ACS})}=N\left\langle\hat{J}_{z}(\phi)\right\rangle_{t_{f}}^{(1)}=\frac{N}{2} \cos \left[\left(\omega_{0}-\omega\right) T\right] .
$$

The propagation of the error in the estimation of the phase $\phi$ is given by (dropping scripts for notational convenience)

$$
\Delta \phi_{\mathrm{ACS}}=\frac{\Delta \hat{J}_{z}(\phi)}{\left|\partial_{\phi}\left\langle\hat{J}_{z}(\phi)\right\rangle\right|}=\frac{1}{\sqrt{N}}
$$

showing that the best sensitivity one can obtain with entangled atoms in the SQL. In terms of the transition frequency, the error is given by $\Delta \omega_{0}=1 /(T \sqrt{N})$. Before closing this section, it is worth summarizing the operator sequence required to implement Ramsey spectroscopy which we do here in terms of an arbitrary initial state $\mid$ in $\rangle$. In the Schröinger picture, this amounts to writing the final state in terms of the initial as

$$
\left.\left|\psi_{f}(\phi)\right\rangle=e^{-i \frac{\pi}{2} \hat{J}_{y}} e^{-i \phi \hat{J}_{z}} e^{-i \frac{\pi}{2} \hat{J}_{y}} \mid \text { in }\right\rangle,
$$

where once again $\phi=\left(\omega_{0}-\omega\right) T$. It is worth noting that Ramsey spectroscopy is mathematically equivalent to optical interferometry in that both can be described through the Lie algebra of SU(2) (see Appendix A). However, the transformations of Eq. 12 is slightly different from how we will describe an interferometer in Section IV.

Now we suppose that we have $N$ atoms prepared in a maximally entangled state of the form

$$
\left|\Psi_{M}\right\rangle=\frac{1}{\sqrt{2}}\left(|e\rangle^{\otimes N}+|g\rangle^{\otimes N}\right)=\frac{1}{\sqrt{2}}(|j, j\rangle+|j,-j\rangle) .
$$

To implement the use of this state for Ramsey spectroscopy as described by the sequence of operators in Eq. 12 , we should take the actual initial state to be $\mid$ in $\rangle=e^{+i \frac{\pi}{2} \hat{J}_{y}}\left|\Psi_{M}\right\rangle$. After a free evolution time $T$ our state is now

$$
\left|\Psi_{M}\right\rangle_{T}=\frac{1}{\sqrt{2}}\left(e^{-i N \phi / 2}|j, j\rangle+e^{i N \phi / 2}|j,-j\rangle\right)
$$

After the second $\pi / 2$ pulse, we arrive at the final state

$$
\begin{aligned}
& |\Psi\rangle_{t_{f}}=\frac{1}{\sqrt{2}}\left(e^{-i N \phi / 2} e^{-i \frac{\pi}{2} \hat{y}_{y}}|j, j\rangle+e^{i N \phi / 2} e^{-i \frac{\pi}{2} \hat{J_{y}}}|j,-j\rangle\right), \\
& =\frac{1}{2^{j} \sqrt{2}} \sum_{m=-j}^{j}\left(\begin{array}{c}
2 j \\
j+m
\end{array}\right)^{1 / 2}\left(e^{-i N \phi / 2}+(-1)^{j+m} e^{i N \phi / 2}\right)|j, m\rangle,
\end{aligned}
$$

where we have used the fact that $e^{-i \frac{\pi}{2} \hat{J}_{y}}|j, \pm j\rangle$ are atomic coherent states generated from different extremal, or fiducial, states $|j, j\rangle$ and $|j,-j\rangle$ (see Appendix A 1 a and B for more detail). The expression of Eq. 15 is not particularly informative when it comes to the evaluation of $\left\langle\hat{J}_{z}(\phi)\right\rangle_{M}$ as it can be shown that

$$
\begin{aligned}
\left\langle\hat{J}_{z}(\phi)\right\rangle_{t_{f}} & ={ }_{T}\left\langle\Psi_{M}\left|e^{i \frac{\pi}{2} \hat{J}_{y}} \hat{J}_{z} e^{-i \frac{\pi}{2} \hat{J}_{y}}\right| \Psi_{M}\right\rangle_{T} \\
& ={ }_{T}\left\langle\Psi_{M}\left|\left(-\hat{J}_{x}\right)\right| \Psi_{M}\right\rangle_{T}=0 .
\end{aligned}
$$

Thus this expectation value furnishes no information on the phase $\phi$.

To address this issue, Bollinger et al..$^{7}$ proposed measuring the quantity $(-1)^{N_{e}}$ where $N_{e}$ is the number of atoms in the excited state. As an operator for a given $j$ value, this reads $\hat{\Pi}_{j}=(-1)^{j+\hat{J}_{z}}=e^{i \pi\left(j+\hat{J}_{z}\right)}$. This requires us to calculate

$$
\begin{aligned}
\left\langle\hat{\Pi}_{j}\right\rangle_{t_{f}} & ={ }_{T}\left\langle\Psi_{M}\left|e^{i \frac{\pi}{2} \hat{J}_{y}} e^{i \pi\left(j+\hat{J}_{z}\right)} e^{-i \frac{\pi}{2} \hat{J}_{y}}\right| \Psi_{M}\right\rangle_{T} \\
& =(-1)^{j}{ }_{T}\left\langle\Psi_{M}\left|e^{-i \pi \hat{J}_{x}}\right| \Psi_{M}\right\rangle_{T} \\
& =(-1)^{j}{ }_{T}\left\langle\Psi_{M}\left|e^{i \frac{\pi}{2} \hat{J}_{z}} e^{-i \pi \hat{J}_{y}} e^{-i \frac{\pi}{2} \hat{J}_{z}}\right| \Psi_{M}\right\rangle_{T},
\end{aligned}
$$

where we have used the relations $e^{i \frac{\pi}{2} \hat{J}_{y}} \hat{J}_{z} e^{-i \frac{\pi}{2} \hat{J}_{y}}=-\hat{J}_{x}$ and $e^{i \frac{\pi}{2} \hat{J}_{z}} \hat{J}_{y} e^{-i \frac{\pi}{2} \hat{J}_{z}}=\hat{J}_{x}$. Setting $\hat{U}=e^{i \frac{\pi}{2} \hat{\hat{J}}_{z}} e^{-i \pi \hat{J}_{y}} e^{-i \frac{\pi}{2} \hat{J}_{z}}$ we have

$$
\begin{gathered}
\left\langle\hat{\Pi}_{j}\right\rangle_{t_{f}}=(-1)^{j} \frac{1}{2}[\langle j, j|\hat{U}| j, j\rangle+\langle j,-j|\hat{U}| j,-j\rangle+ \\
\left.+e^{i N \phi}\langle j, j|\hat{U}| j,-j\rangle+e^{-i N \phi}\langle j,-j|\hat{U}| j, j\rangle\right]
\end{gathered}
$$




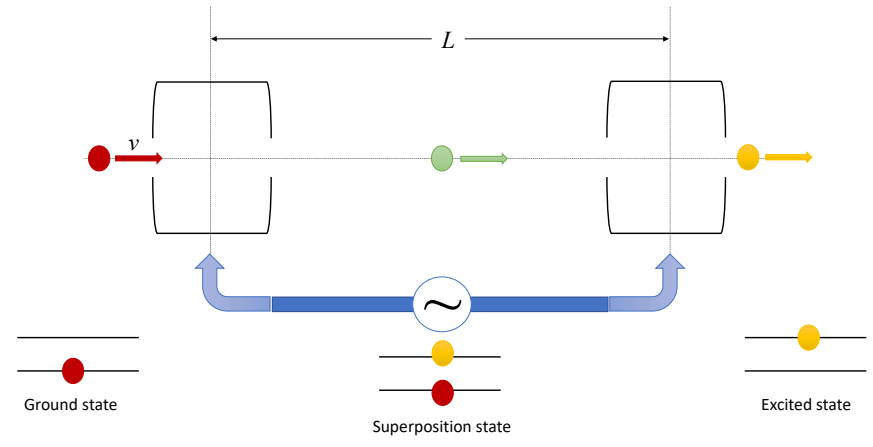

FIG. 2. A sketch of the Ramsey technique employed for the case of a single atom initially prepared in the ground state. The first $\pi / 2$ pulse (frequency $\omega$ ) places the atom in a superposition state. The free evolution over cavity length $L$ results in a phase shift $\phi=\left(\omega_{0}-\omega\right) T$ ( $T=L / v$, where $v$ is the atom velocity). The final $\pi / 2$ pulse (frequency $\omega$ ) places the state of the atom near the excited state.

Then with

$$
\begin{gathered}
\langle j, j|\hat{U}| j, j\rangle=d_{j, j}^{j}(\pi)=0, \\
\langle j,-j|\hat{U}| j,-j\rangle=d_{-j,-j}^{j}(\pi)=0, \\
\langle j, j|\hat{U}| j,-j\rangle=(-1)^{j} d_{j,-j}^{j}(\pi)=(-1)^{j}, \\
\langle j,-j|\hat{U}| j, j\rangle=(-1)^{-j} d_{-j, j}^{j}(\pi)=(-1)^{j},
\end{gathered}
$$

where $d_{m^{\prime}, m}^{j}(\beta)$ are the Wigner- $d$ matrix elements discussed in Appendix B, we finally have

$$
\left\langle\hat{\Pi}_{j}\right\rangle_{t_{f}}=(-1)^{N} \cos \left[N\left(\omega_{0}-\omega\right) T\right] .
$$

Note the appearance of the factor of $N$ in the argument of the cosine. This is the consequence of the maximal entanglement between the $N$ atoms, consequently leading to an increase in the sensitivity of the frequency measurement by a factor of $1 / \sqrt{N}$ over the SQL. Again, setting $\phi=\left(\omega_{0}-\omega\right) T$, the error propagation calculus in this case results in

$$
\Delta \phi_{M}=\frac{\Delta \hat{\Pi}_{j}}{\left|\partial_{\phi}\left\langle\hat{\Pi}_{j}\right\rangle_{t_{f}}\right|}=\frac{1}{N}
$$

or that $\Delta \omega_{0}=1 / N T$. This is considered a Heisenberg-limited uncertainty as it scales with the inverse of $N$. A scheme for the generation of the maximally entangled state of Eq. 13 was discussed by Bollinger et al. ${ }^{7}$ and a different scheme was discussed by Steinbach and Gerry 11 . An experimental realization of parity-measurements has been performed by Leibfried et $a l^{[12}$ with three trapped ions prepared in a maximally entangled state.

In the next section we will briefly review the basics of phase estimation and make the connection between parity-based measurement and the minimum phase uncertainty: the quantum Cramér-Rao bound.

\section{PHASE ESTIMATION AND THE QUANTUM CRAMÉR-RAO BOUND}

Phases cannot be measured, only approximated. This is due to the fact that there exists no Hermitian phase operator ${ }^{13}$. Consequently, within the realm of quantum mechanics, the phase is treated as a classical parameter rather than a quantum observable. The general approach to interferometry, be it optical or atomic, is to encode a suitable 'probe state' with a classically-treated phase and determine the optimal detection observable for estimating its value. The art lies in determining the best combination of probe state and detection observable that yields the highest resolution and smallest phase uncertainty. In what follows, we will discuss some of the basics of phase estimation and arrive at a relation between parity-based detection and the upper-bound on phase estimation, which determines the greatest sensitivity afforded to a given quantum state.

\section{A. The Cramér-Rao lower bound}

In the broadest sense, an interferometer is an apparatus that can transform an input 'probe state' $\hat{\rho}$ in a manner such that the transformation can be parametrized by a real, unknown, parameter $\varphi$. A measurement is then performed on the output state $\hat{\rho}(\varphi)$ from which an estimation of the parameter $\varphi$ takes place. The most general formulation of a measurement in quantum theory is a positive-operator valued measure (POVM). A POVM consists of a set of non-negative Hermitian operators satisfying the unity condition $\sum_{\varepsilon} \hat{E}(\varepsilon)=1$. Following the work of Pezzé et al.14, the conditional probability to observe the result $\varepsilon$ for a given value $\varphi$, known as the likelihood, is

$$
P(\varepsilon \mid \varphi)=\operatorname{Tr}[\hat{E}(\varepsilon) \hat{\rho}(\varphi)]
$$

If the input state is made up of $m$ independent uncorrelated subsystems such that $\hat{\rho}=\hat{\rho}^{(1)} \otimes \hat{\rho}^{(2)} \otimes \hat{\rho}^{(3)} \otimes \hat{\rho}^{(4)} \otimes \ldots \otimes \hat{\rho}^{(m)}$ and we restrict ourselves to local operations such that the phase $\varphi$ is encoded into each subsystem and assuming independent measurements are performed on each, then the likelihood function becomes the product of the single-measurement probabilities

$$
P(\varepsilon \mid \varphi)=\prod_{i=1}^{m} P_{i}\left(\varepsilon_{i} \mid \varphi\right)
$$

where $P_{i}\left(\varepsilon_{i} \mid \varphi\right)=\operatorname{Tr}\left[\hat{E}^{(i)}\left(\varepsilon_{i}\right) \hat{\rho}^{(i)}(\varphi)\right]$. For the case of independent measurements, as described in Eq. 23, often one considers the log-Likelihood function

$$
L(\varepsilon \mid \varphi) \equiv \ln P(\varepsilon \mid \varphi)=\sum_{i=1}^{m} \ln P_{i}\left(\varepsilon_{i} \mid \varphi\right) .
$$

We define the estimator $\Phi(\varepsilon)$ as any mapping of a given set of outcomes, $\varepsilon$, onto parameter space in which an estimation 
of the phase is made. A prevalent example is the maximum-

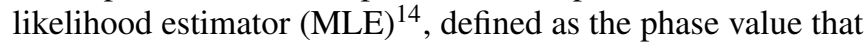
maximizes the likelihood function

$$
\Phi_{\mathrm{MLE}}(\varepsilon)=\arg \left[\max _{\varphi} P(\varepsilon \mid \varphi)\right] .
$$

An estimator can be characterized by its phase dependent mean value

$$
\langle\Phi\rangle_{\varphi}=\sum_{\varepsilon} P(\varepsilon \mid \varphi) \Phi(\varepsilon)
$$

and its variance

$$
(\Delta \Phi)_{\varphi}^{2}=\sum_{\varepsilon} P(\varepsilon \mid \varphi)\left[\Phi(\varepsilon)-\langle\Phi\rangle_{\varphi}\right]^{2} .
$$

We will now discuss what it means for an estimator to be 'good', which in this case, refers to an estimator that provides the smallest uncertainty. These estimators are known as unbiased estimators, and are defined as estimators whose average value coincides with the true value of the parameter in question, that is $\langle\Phi(\varepsilon)\rangle_{\varphi}=\varphi$ is true for all values of the parameter $\varphi$ while estimators that are unbiased in the limit of $m \rightarrow \infty$, such as the MLE, are considered asymptotically consistent. Estimators not satisfying this condition are considered biased while estimators that are unbiased for a certain range of the parameter $\varphi$ are considered locally unbiased.

We now move on to perhaps one of the most important tools in the theory of phase estimation: the Cramér-Rao bound (CRB). The CRB serves to set a lower bound on the variance of any arbitrary estimator and is given formally as 5

$$
\left(\Delta \Phi_{\mathrm{CRB}}\right)_{\varphi}^{2}=\frac{\left(\frac{\partial\langle\Phi\rangle_{\varphi}}{\partial \varphi}\right)^{2}}{F(\varphi)} \leq(\Delta \Phi)_{\varphi}^{2},
$$

where the quantity $F(\varphi)$ is the classical Fisher information (FI), given by

$$
F(\varphi) \equiv\left\langle\left(\frac{\partial L(\varepsilon \mid \varphi)}{\partial \varphi}\right)^{2}\right\rangle=\sum_{\varepsilon} \frac{1}{P(\varepsilon \mid \varphi)}\left(\frac{\partial P(\varepsilon \mid \varphi)}{\partial \varphi}\right)^{2}
$$

where the sum extends over all possible values of the measurement values, $\varepsilon$. While Eq. 28 is the most general form the CRB, it is most useful for the cases of unbiased estimators where the numerator on the right-hand side, $\partial_{\varphi}\langle\Phi\rangle_{\varphi}=1$. For this case, the CRB is simply given as the inverse of the Fisher information $F(\varphi)$. An estimator that saturates the CRB is said to be efficient. The existence, however, of an efficient estimator depends on the properties of the probability distribution. It is also worth noting that the derivation of the Fisher information of Eq. 29 assumed an initial state $\rho$ comprised of $m$-independent subsystems. It is straight forward to show the additivity of the Fisher information $F(\varphi)=\sum_{i=1}^{m} F^{(i)}(\varphi)$ using Eq. 29 and plugging in Eq. 23 and 24 where $F^{(i)}(\varphi)$ is the Fisher information of the $i^{\text {th }}$ subsystem. For $m$ identical subsystems and measurements, this yields $F(\varphi)=m F^{(1)}(\varphi)$, where $F^{(1)}(\varphi)$ is the Fisher information for a single-measurement and $m$ is the total number of measurements. This is the form of the Fisher information most often used in the literature.

\section{B. Quantum Fisher information and the upper bound}

We now turn our attention towards discussing an upper bound $\sqrt{16}$ on phase estimation, known as the quantum CramérRao bound (qCRB), which in turn will be dependent on the quantum Fisher information $F_{Q}(\mathrm{QFI})$. We obtain this upper bound by maximizing the FI over all possible POVMs 17118 ,

$$
F_{\mathrm{Q}}[\hat{\rho}(\varphi)] \equiv \max _{\{\hat{E}(\varepsilon)\}} F[\hat{\rho}(\varphi),\{\hat{E}(\varepsilon)\}]
$$

where this quantity is known as the quantum Fisher information. It is important to note that the quantum Fisher information is independent of the POVM used. This quantity can be expressed as 19

$$
F_{\mathrm{Q}}[\hat{\rho}(\varphi)]=\operatorname{Tr}\left[\hat{\rho}(\varphi) \hat{L}_{\varphi}^{2}\right]
$$

where $\hat{L}_{\varphi}$ is known as the symmetric logarithmic derivative (SLD ${ }^{\sqrt{20}}$ defined as the solution to the equation

$$
\frac{\partial \hat{\rho}(\varphi)}{\partial \varphi}=\frac{\hat{\rho}(\varphi) \hat{L}_{\varphi}+\hat{L}_{\varphi} \hat{\rho}(\varphi)}{2}
$$

The chain of inequalities is now

$$
(\Delta \Phi)_{\varphi}^{2} \geq(\Delta \Phi)_{\mathrm{CRB}}^{2} \geq(\Delta \Phi)_{\mathrm{qCRB}}^{2},
$$

where it follows that the quantum Cramér-Rao bound (also known as the Helstrom bound ${ }^{21}$ ) is given by

$$
(\Delta \Phi)_{\mathrm{qCRB}}^{2} \equiv \frac{\left(\frac{\partial\langle\Phi\rangle}{\partial \varphi}\right)^{2}}{F_{\mathrm{Q}}[\hat{\rho}(\varphi)]} .
$$

Since the qCRB is inversely proportional to the QFI and the QFI itself is a maximization over all possible POVMs, it is clear to see how the qCRB serves as an upper bound on phase estimation.

\section{Calculating the QFI for pure and mixed states}

Here we work through a suitable expression for the QFI, using our definition of the SLD given in Eq. 32, in terms of 
the complete basis $\{|n\rangle\}$, where our density operator is now given generally as $\hat{\rho}(\varphi)=\sum_{n} p_{n}|n\rangle\langle n|$. Following the work of Pezzé et al ${ }^{14}$, the quantum Fisher information can be written in this basis as

$$
\begin{aligned}
F_{\mathrm{Q}}[\hat{\rho}(\varphi)] & =\operatorname{Tr}\left[\hat{\rho}(\varphi) \hat{L}_{\varphi}^{2}\right]=\sum_{k, k^{\prime}} p_{k}\left|\left\langle k\left|\hat{L}_{\varphi}\right| k^{\prime}\right\rangle\right|^{2} \\
& =\sum_{k, k^{\prime}} \frac{p_{k}+p_{k^{\prime}}}{2} \times\left|\left\langle k\left|\hat{L}_{\varphi}\right| k^{\prime}\right\rangle\right|^{2} .
\end{aligned}
$$

Thus it is sufficient to know the matrix elements of the SLD, $\left\langle k\left|\hat{L}_{\varphi}\right| k^{\prime}\right\rangle$ in order to calculate the QFI. Using Eq. 32 and our general density operator, it is easy to show

$$
\left\langle k\left|\hat{L}_{\varphi}\right| k^{\prime}\right\rangle=\left(\frac{2}{p_{k}+p_{k^{\prime}}}\right) \times\left\langle k\left|\partial_{\varphi} \hat{\rho}(\varphi)\right| k^{\prime}\right\rangle,
$$

which makes Eq. $35^{22}$

$$
F_{\mathrm{Q}}[\hat{\rho}(\varphi)]=\sum_{k, k^{\prime}} \frac{2}{p_{k}+p_{k^{\prime}}} \times\left|\left\langle k\left|\partial_{\varphi} \hat{\rho}(\varphi)\right| k^{\prime}\right\rangle\right|^{2}
$$

We proceed further through the use of the definition

$$
\partial_{\varphi} \hat{\rho}(\varphi)=\sum_{k}\left(\partial_{\varphi} p_{k}\right)|k\rangle\left\langle k\left|+\sum_{k} p_{k}\right| \partial_{\varphi} k\right\rangle\left\langle k\left|+\sum_{k} p_{k}\right| k\right\rangle\left\langle\partial_{\varphi} k\right|,
$$

which is a simple application of the chain rule for derivatives. Using the identity $\partial_{\varphi}\left\langle k \mid k^{\prime}\right\rangle=\left\langle\partial_{\varphi} k \mid k^{\prime}\right\rangle+\left\langle k \mid \partial_{\varphi} k^{\prime}\right\rangle \equiv 0$, the matrix elements in Eq. 37 become

$$
\left\langle k\left|\partial_{\varphi} \hat{\rho}(\varphi)\right| k^{\prime}\right\rangle=\left(\partial_{\varphi} \hat{\rho}(\varphi)\right) \delta_{k, k^{\prime}}+\left(p_{k}-p_{k^{\prime}}\right)\left\langle\partial_{\varphi} k \mid k^{\prime}\right\rangle .
$$

The SLD and QFI then become

$$
\begin{aligned}
& \hat{L}_{\varphi}=\sum_{k} \frac{\partial_{\varphi} p_{k}}{p_{k}} \times|k\rangle\left\langle k\left|+2 \sum_{k, k^{\prime}} \frac{p_{k}-p_{k^{\prime}}}{p_{k}+p_{k^{\prime}}} \times\left\langle\partial_{\varphi} k \mid k^{\prime}\right\rangle\right| k^{\prime}\right\rangle\langle k|, \\
& F_{\mathrm{Q}}[\hat{\rho}(\varphi)]=\sum_{k} \frac{\left(\partial_{\varphi} p_{k}\right)^{2}}{p_{k}}+2 \sum_{k, k^{\prime}} \frac{\left(p_{k}-p_{k^{\prime}}\right)^{2}}{p_{k}+p_{k^{\prime}}} \times\left|\left\langle\partial_{\varphi} k \mid k^{\prime}\right\rangle\right|^{2},
\end{aligned}
$$

respectively. These results, we show next, simplify in the case of pure states where we can write $\hat{\rho}(\varphi)=|\psi(\varphi)\rangle\langle\psi(\varphi)|$ and consequently $\partial_{\varphi} \hat{\rho}(\varphi)=\partial_{\varphi} \hat{\rho}^{2}(\varphi)=\hat{\rho}(\varphi)\left[\partial_{\varphi} \hat{\rho}(\varphi)\right]+$ $\left[\partial_{\varphi} \hat{\rho}(\varphi)\right] \hat{\rho}(\varphi)$. Using this, and a cursory glance at Eq. 32 , it is clear the SLD becomes

$$
\begin{aligned}
\hat{L}_{\varphi} & =2\left[\partial_{\varphi} \hat{\rho}(\varphi)\right]=2\left[\partial_{\varphi}|\psi(\varphi)\rangle\langle\psi(\varphi)|\right] \\
& =2\left|\partial_{\varphi} \psi\right\rangle\langle\psi|+2| \psi\rangle\left\langle\partial_{\varphi} \psi\right|
\end{aligned}
$$

where in the last step, the $\varphi$-dependency of $|\psi\rangle$ is implicit for notational convenience. Plugging this directly into the first line of Eq. 35 yields

$$
\begin{aligned}
F_{\mathrm{Q}}[\hat{\rho}(\varphi)] & =\operatorname{Tr}\left[\hat{\rho}(\varphi) \hat{L}_{\varphi}^{2}\right]=\left\langle\psi\left|\hat{L}_{\varphi}^{2}\right| \psi\right\rangle \\
& =4\left\{\left\langle\partial_{\varphi} \psi \mid \partial_{\varphi} \psi\right\rangle-\left|\left\langle\partial_{\varphi} \psi \mid \psi\right\rangle\right|^{2}\right\}
\end{aligned}
$$

which is the form of the QFI most often used in quantum metrology literature. Next we move on to discussing a specific detection observable: photon number parity.

\section{Connection to parity-based detection}

The central theme discussed throughout this paper is the use of the quantum mechanical parity operator as a detection observable in quantum optical interferometry. The use of parity as a detection observable first came about in conjunction with high precision spectroscopy, by Bollinger et al. ${ }^{7}$. It was first adapted and formally introduced for use in quantum optical

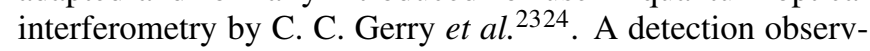
able is said to be optimal if for a given state, the CRB achieves the qCRB, that is,

$$
(\Delta \Phi)_{\varphi}^{2} \geq(\Delta \Phi)_{\mathrm{CRB}}^{2}=(\Delta \Phi)_{\mathrm{qCRB}}^{2}
$$

Furthermore, parity detection achieves maximal phase sensitivity at the qCRB for all pure states that are path symmetric $^{25 \mid 26}$. For the purposes of this paper, it is sufficient to derive the classical Fisher information. We start from the expression for the classical Fisher information, assuming a single measurement is performed, given in Eq. 29. For parity 27 , the measurement outcome $\varepsilon$ can either be positive + or negative - and satisfies $P(+\mid \varphi)+P(-\mid \varphi) \equiv 1$. The expectation value of the parity operator can then be expressed as a sum over the possible eigenvalues weighted with the probability of that particular outcome leading to

$$
\begin{aligned}
\langle\hat{\Pi}\rangle & =\sum_{i} P(i \mid \varphi) \lambda_{i}=P(+\mid \varphi)-P(-\mid \varphi) \\
& =2 P(+\mid \varphi)-1=1-2 P(-\mid \varphi) .
\end{aligned}
$$

Likewise, we can calculate the variance

$$
\begin{aligned}
(\Delta \hat{\Pi})^{2} & =\left\langle\hat{\Pi}^{2}\right\rangle-\langle\hat{\Pi}\rangle^{2}=1-\langle\hat{\Pi}\rangle^{2} \\
& =1-(P(+\mid \varphi)-P(-\mid \varphi))^{2} \\
& =1-(P(+\mid \varphi)+P(-\mid \varphi))^{2}+4 P(+\mid \varphi) P(-\mid \varphi) \\
& =4 P(+\mid \varphi) P(-\mid \varphi) .
\end{aligned}
$$

Finally, from Eq. 44, it follows that

$$
\frac{\partial P(+\mid \varphi)}{\partial \varphi}=\frac{1}{2} \frac{\partial\langle\hat{\Pi}\rangle}{\partial \varphi}=-\frac{\partial P(-\mid \varphi)}{\partial \varphi} .
$$

Combining Eq. 45 and 46 we find 


$$
\begin{aligned}
F^{(1)}(\varphi) & =\sum_{\varepsilon_{1}} \frac{1}{P\left(\varepsilon_{1} \mid \varphi\right)}\left(\frac{\partial P\left(\varepsilon_{1} \mid \varphi\right)}{\partial \varphi}\right)^{2} \\
& =\frac{1}{(\Delta \hat{\Pi})^{2}}\left|\frac{\partial\langle\hat{\Pi}\rangle}{\partial \varphi}\right|^{2}
\end{aligned}
$$

making the CRB / qCRB

$$
\Delta \Phi_{\mathrm{CRB} / \mathrm{q} C R B}=\frac{\sqrt{1-\langle\hat{\Pi}\rangle^{2}}}{\left|\partial_{\varphi}\langle\hat{\Pi}\rangle\right|}=\frac{1}{\sqrt{F^{(1)}(\varphi)}} .
$$

Eq. 48 is simply the phase uncertainty obtained through the error propagation calculus. This is advantageous over other means of detection, such as photon-number counting, because the use of parity does not require any pre- or postdata processing. By comparison, photon number counting typically works by construction of a phase probability distribution conditioned on the outcome of a sequence of $m$ measurements 28 . After a sequence of detection events, the error in the phase estimate is determined from this distribution. While this provides phase estimation at the qCRB, it lacks the advantage of being directly determined from the signal, unlike the use of parity 27 . There are disadvantages to using parity, however. Performance of photon number parity is highly susceptible to losses. Parity also achieves maximal phase sensitivities at particular values of the phase, restricting its use to estimating local phases ${ }^{29}$. Restricting its use to local parameter estimation, however, is not terribly problematic in interferometry, as one is interested in measuring small changes to parameters that are more-or-less known.

It is worth pointing out that the optimal POVM depends, in general, on $\varphi$. This is somewhat problematic as it requires one to already know the value of the parameter $\varphi$ in order to choose an optimal estimator. Some work has been done to overcome this obstacle ${ }^{30}$ which concludes the QFI can be asymptotically obtained in a number of measurements without any knowledge of the parameter. For all cases considered throughout this paper, we will use parity as our detection observable (except in cases where we wise to draw comparisons between observables), which we know saturates the qCRB. We will now move on to discuss how one calculates the QFI in quantum optical interferometry.

\section{Calculating the QFI in quantum optical interferometry}

We use the Schwinger realization of the su(2) algebra with two sets of boson operators, discussed in detail in Appendix A 2 to describe a standard Mach-Zehnder interferometer ${ }^{8}$. In this realization, the quantum mechanical beam splitter can be viewed as a rotation about a given (fictitious) axis determined by the choice of angular momentum operator, i.e. the choice of a $\hat{J}_{x}$-operator performs a rotation about the $x$-axis while the choice of a $\hat{J}_{y}$-operator performs a rotation about the $y$-axis.
An induced phase shift, assumed to be in the $b$-mode, is described by a rotation about the $z$-axis described by the use of the $\hat{J}_{z}$-operator. The state just before the second beam splitter is given as

$$
|\psi(\varphi)\rangle=e^{-i \varphi \hat{J}_{z}} e^{-i \frac{\pi}{2} \hat{J}_{x}}|\mathrm{in}\rangle,
$$

where we are assuming the beam splitters to be 50:50. This in turn makes the derivative

$$
\left.\left|\psi^{\prime}(\varphi)\right\rangle=-i e^{-i \varphi \hat{J}_{z}} \hat{J}_{z} e^{-i \frac{\pi}{2} \hat{J}_{x}} \mid \text { in }\right\rangle
$$

leading to

$$
\left\langle\psi^{\prime}(\varphi) \mid \psi(\varphi)\right\rangle=i\left\langle\text { in }\left|\hat{J}_{y}\right| \text { in }\right\rangle,
$$

and

$$
\left\langle\psi^{\prime}(\varphi) \mid \psi^{\prime}(\varphi)\right\rangle=\left\langle\text { in }\left|\hat{J}_{y}^{2}\right| \text { in }\right\rangle,
$$

where we have made use of the Baker-Hausdorf identity in simplifying

$$
e^{i \frac{\pi}{2} \hat{J}_{x}} \hat{J}_{z} e^{-i \frac{\pi}{2} \hat{J}_{x}}=\hat{J}_{y}
$$

Combining Eq. 51 and 52 into Eq. 42 yields for the QFI

$$
\begin{aligned}
F_{\mathrm{Q}}[\hat{\rho}(\varphi)] & =4\left\{\left\langle\partial_{\varphi} \psi \mid \partial_{\varphi} \psi\right\rangle-\left|\left\langle\partial_{\varphi} \psi \mid \psi\right\rangle\right|^{2}\right\} \\
& =4\left\langle\left(\Delta \hat{J}_{y}\right)^{2}\right\rangle_{\text {in }}
\end{aligned}
$$

which is simply the variance of the $\hat{J}_{y}$-operator with respect to the initial input state $\mid$ in $\rangle$. This is the form of the QFI used in all of the following interferometric calculations. One important thing to notice is that the quantum Fisher information depends solely on the initial state and not on the value of the phase $\varphi$ to be measured.

Note that Eq. 54 is a general result. Let $\hat{\mathscr{O}}$ be a generator of a flow parameterized by $\zeta$ such that the wave function evolves according to the Schrödinger equation $i \partial_{\zeta}|\psi\rangle=\hat{\mathscr{O}}|\psi\rangle$ with solution $|\psi(\zeta)\rangle=e^{-i \hat{\mathscr{O}} \zeta}|\psi(0)\rangle$. Then $\left|\partial_{\zeta} \psi\right\rangle=-i \hat{\mathscr{O}}|\psi\rangle$ so that $\left\langle\partial_{\zeta} \psi \mid \partial_{\zeta} \psi\right\rangle=\left\langle\hat{\mathscr{O}}^{2}\right\rangle$, and $\left|\left\langle\psi \mid \partial_{\zeta} \psi\right\rangle\right|^{2}=\langle\hat{\mathscr{O}}\rangle^{2}$. Hence $F_{Q}(\zeta)=4\left[\left\langle\partial_{\zeta} \psi \mid \partial_{\zeta} \psi\right\rangle-\left|\left\langle\psi \mid \partial_{\zeta} \psi\right\rangle\right|^{2}\right]=4\left\langle(\Delta \hat{\mathcal{O}})^{2}\right\rangle_{\zeta}$. Thus, both Eq.28 and Eq. 34 yield a Fourier-like uncertainty relation for unbiased estimators of the form $(\Delta \Phi)_{\zeta}\langle(\Delta \hat{\mathscr{O}})\rangle_{\zeta} \geq 1 / 2$ (if we do not use the maximum FI) reminiscent of $\Delta x \Delta k \geq 1 / 2$, so that a precise measurement $(\Delta \Phi)_{\zeta} \ll 1 / 2$ of $\zeta$ requires a large uncertainty (variance) $\langle(\Delta \hat{\mathscr{O}})\rangle_{\zeta} \gg 1 / 2$ in its generation. Noting that $\hat{\Pi}_{b}=(-1)^{\hat{b}^{\dagger} \hat{b}}=e^{i \pi \hat{n}_{b}}$ is both unitary as well as Hermitian, define $\hat{\Pi}_{b}^{(\phi)} \equiv e^{-i \phi \hat{n}_{b}}$ with $\hat{\mathscr{O}} \rightarrow \hat{n}_{b}$ and $\zeta \rightarrow \phi$ (which we take to be $\phi=-\pi$ for the parity operator). Thus, a 


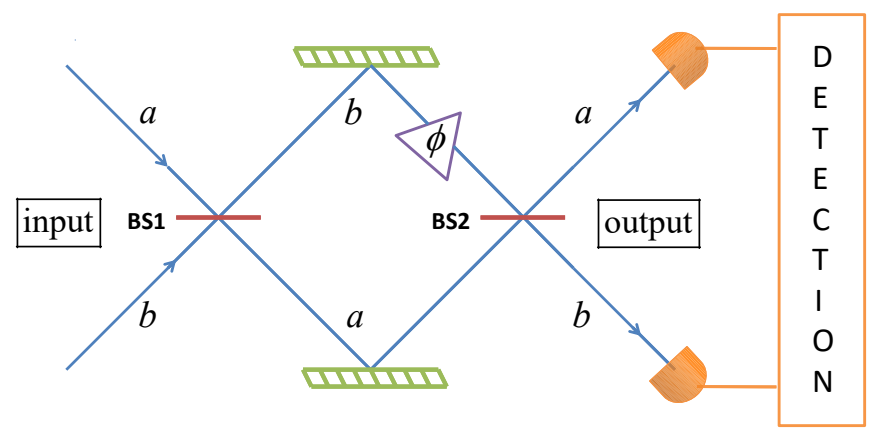

FIG. 3. A sketch of a standard Mach-Zehnder interferometer (MZI). Light propagates through one (or both) input ports of the MZI. A phase shift occurs after the first beam splitter due to a relative path length difference between the two arms of the MZI. Measurement occurs on one (or both) modes of the output.

measurement $\hat{\Pi}_{b}^{(\phi)}|\psi(0)\rangle=e^{-i \phi \hat{n}_{b}}|\psi(0)\rangle$ can be thought of as a Schrödinger-like evolution in $\phi$ with generator $\hat{n}_{b}$. Then, from above $F_{\Pi_{b}}(\phi)=4\left\langle\left(\Delta \hat{n}_{b}\right)^{2}\right\rangle_{\phi}$. This result is independent of the parameter $\phi$. This leads to the insightful interpretation of Eq. 48 as $\Delta \Phi_{\mathrm{CRB} / \mathrm{qCRB}} \Delta \hat{n}_{b}=\frac{1}{2}$, i.e. a more formal statement of the classical number-phase uncertainty relationship $\Delta \phi \Delta N \geq \frac{1}{2}$. In fact, we now see that the parity operator $\hat{\Pi}_{b}$ leads to the minimal phase uncertainty relationship possible, since it saturates the inequality.

Next we will discuss the phase uncertainty and measurement resolutions obtained using parity-based detection in interferometry for a number of cases in which input classical and/or quantum mechanical states of light are considered.

\section{QUANTUM OPTICAL INTERFEROMETRY}

In this section we highlight several different interferometric schemes involving both classical and non-classical states of light comparing the use of several different detection observables. Here we show that in cases where the bound on phase sensitivity is not saturated, parity-based detection yields sub-SQL limited phase sensitivity and can, in certain cases, approach or out-perform the HL of phase sensitivity. We also draw attention to the correlation between parity-based detection and the saturation of the qCRB. Before we get into certain cases, we will provide the reader with a concise derivation of the output state of an interferometer for arbitrary initial states as well as the average value of an arbitrary detection observable and the subsequent phase uncertainty.

Consider an interferometer transforming an initial state $\mid$ in $\rangle$ according to

$$
\left.\left.\mid \text { out }\rangle=e^{i \frac{\pi}{2} \hat{J}_{x}} e^{-i \phi \hat{J}_{z}} e^{-i \frac{\pi}{2} \hat{J}_{x}} \mid \text { in }\right\rangle=e^{-i \phi \hat{J}_{y}} \mid \text { in }\right\rangle,
$$

where we employ the Schwinger realization of the SU(2) Lie algebra (see Appendix A2). It is worth comparing the expression for the output state of Eq. 55 with that of the final state obtained when one performs Ramsey spectroscopy, as shown in Eq. 12. The $\pi / 2$ pulses performed on atomic states is analogous to a beam splitter transformation affecting two boson modes in that both can be described in terms of the $\mathrm{su}(2)$ Lie algebra. The same can be said of how the phase $\phi$ is encoded in both procedures, though they have very different physical interpretations. For atomic systems, the phase shift arises during the period of free evolution while in interferometry it stems from a relative path length difference between the two arms of the interferometer.

For the most general of separable initial states, $\mid$ in $\rangle=$ $\left|\psi_{0}^{(1)}\right\rangle_{a} \otimes\left|\psi_{0}^{(2)}\right\rangle_{b}$, where in the photon number basis $\left|\psi_{0}^{(i)}\right\rangle=$ $\sum_{n=0}^{\infty} C_{n}^{(i)}|n\rangle, n \in \mathbb{Z}^{+}$, the input state can be expressed as

$$
\begin{aligned}
\mid \text { in }\rangle & =\sum_{n=0}^{\infty} \sum_{q=0}^{\infty} C_{n}^{(1)} C_{q}^{(2)}|n\rangle_{a} \otimes|q\rangle_{b} \\
& =\sum_{j=0,1 / 2, . .}^{\infty} \sum_{m=-j}^{j} C_{j+m}^{(1)} C_{j-m}^{(2)}|j, m\rangle .
\end{aligned}
$$

Working in the Schrödinger picture, the transformation of Eq. 55 acting on this initial state yields the output state

$$
\begin{aligned}
\mid \text { out }\rangle & =\sum_{j^{\prime}=0,1 / 2, \ldots}^{\infty} \sum_{m^{\prime}=-j^{\prime}}^{j^{\prime}}\left\langle j^{\prime}, m^{\prime}\left|e^{-i \phi \hat{J}_{y}}\right| \text { in }\right\rangle\left|j^{\prime}, m^{\prime}\right\rangle \\
& =\sum_{j=0,1 / 2, . .}^{\infty} \sum_{m^{\prime}=-j}^{j} \Gamma_{j, m^{\prime}}(\phi)\left|j+m^{\prime}\right\rangle_{a} \otimes\left|j-m^{\prime}\right\rangle_{b},
\end{aligned}
$$

where we have inserted a complete set of states in the angular momentum basis and where the phase-dependent state coefficients are given by

$$
\Gamma_{j, m^{\prime}}(\phi)=\sum_{m=-j}^{j} C_{j+m}^{(1)} C_{j-m}^{(2)} d_{m^{\prime}, m}^{j}(\phi) .
$$

The phase-dependent term in Eq. 58 are the well known Wigner- $d$ matrix elements discussed in some detail in Appendix B 1. Note that if our initial state is an entangled twomode state, the corresponding coefficients would be of the form $A_{n, p}$ where $A_{n, p} \neq C_{n}^{(1)} C_{p}^{(2)}$. For an arbitrary detection observable $\hat{\mathscr{O}}$, the expectation value can be calculated directly as

$$
\langle\hat{\mathscr{O}}\rangle_{\mathrm{out}}=\sum_{j, m^{\prime}} \sum_{J, M} \Gamma_{J, M}^{*}(\phi) \Gamma_{j, m^{\prime}}(\phi)\left\langle J, M|\hat{\mathscr{O}}| j, m^{\prime}\right\rangle .
$$

From this the phase uncertainty can be found through use of the usual error propagation calculus to be

$$
\Delta \phi_{\hat{O}}=\frac{\Delta \hat{\mathscr{O}}_{\text {out }}}{\left|\partial_{\phi}\langle\hat{\mathscr{O}}\rangle_{\text {out }}\right|} .
$$


From the perspective of the Heisenberg picture, the transformed observable $\hat{\mathscr{O}}^{\prime}$ is given by $\hat{\mathscr{O}}^{\prime}=e^{i \phi \hat{J}_{y}} \hat{\mathscr{O}} e^{-i \phi \hat{J}_{y}}$ and the derivative of its expectation value

$$
\partial_{\phi}\langle\hat{\mathscr{O}}\rangle_{\text {out }}=\partial_{\phi}\left\langle\hat{\mathscr{O}}^{\prime}\right\rangle_{\text {in }}=i\left\langle\left[\hat{J}_{y}, \hat{\mathscr{O}}^{\prime}\right]\right\rangle_{\text {in }} .
$$

We remind the reader that the greatest phase sensitivity afforded by classical states is the standard quantum limit (SQL), $\Delta \phi_{\mathrm{SQL}}=1 / \sqrt{\bar{n}}$ while for quantum states the phase sensitivity is bounded by the Heisenberg limit (HL) $\Delta \phi_{\mathrm{HL}}=1 / \bar{n}$. We note that while the HL serves as a bound on phase sensitivity, it has been demonstrated to be beaten by some quantum states for low (but still $>1$ ) average photon numbers 31 . The goal of this section is to highlight the effectiveness of parity-based measurement performed on one of the output ports. Unless otherwise stated, we assume measurement is performed on the output $b$-mode without loss of generality 32 . We are now ready to discuss several different cases.

\section{A. The N00N states}

The optical $N 00 N$ state has been extensively studied for use in high-precision quantum metrology 24/33/34/35. It is defined as the superposition state in which $N$ photons are in one mode (labeled the $a$-mode) while none are in the either ( $b$-mode) $|N, 0\rangle_{a, b}$ and where no photons are in the $a$-mode and $N$ photons are in the $b$-mode $|0, N\rangle_{a, b}$, and can be written generally as

$$
|\psi\rangle_{N 00 N}=\frac{1}{\sqrt{2}}\left(|N, 0\rangle+e^{i \Phi_{N}}|0, N\rangle\right),
$$

where $\Phi_{N}$ is a relative phase factor that may depend on $N$ and whose value will generally depend on the method of state generation. The origin of the moniker "NOON state" is obvious, though such states are also known as maximally path-entangled number states as the path of the definite number of photons $N$ in the superposition state of Eq. 62 can be interpreted as being objectively indefinite.

Let us consider an interferometric scheme in which the initial state is given by $\mid$ in $\rangle=|N, 0\rangle_{a, b}$ and the first beam splitter is replaced by an optical device that transforms the initial state into the $N 00 N$ state of Eq. 62 (a "magic" beam splitter, so to speak). The state after the phase shift (taken to be in the $b$-mode) is

$$
\left|\psi_{N}(\phi)\right\rangle=\frac{1}{\sqrt{2}}\left(|N, 0\rangle_{a, b}+e^{i N \phi+\Phi_{N}}|0, N\rangle_{a, b}\right),
$$

amounting to an additional relative phase shift of $N \phi$. Finally, the state after the second beam splitter is 24

$$
\mid \text { out }\rangle=\frac{1}{\sqrt{2^{N+1} N !}}\left[\left(\hat{a}^{\dagger}+i \hat{b}^{\dagger}\right)+e^{i\left(N \phi+\Phi_{N}\right)}\left(\hat{b}^{\dagger}+i \hat{a}^{\dagger}\right)\right]|0,0\rangle_{a, b},
$$

which for the case of $N=1$ yields the same phase sensitivity as the $\mid$ in $\rangle=|1,0\rangle_{a, b}$ state when implementing intensitydifference measurements in a regular MZI scheme. For the case of $N=2$, the output state can be written as

$$
\begin{aligned}
\mid \text { out }, 2\rangle= & \frac{1}{2 \sqrt{2}}\left[\left(1-e^{i 2 \phi+\Phi_{2}}\right)|2,0\rangle_{a, b}+\right. \\
& \left.+\sqrt{2} i\left(1+e^{i 2 \phi+\Phi_{2}}\right)|1,1\rangle_{a, b}+\left(e^{i 2 \phi+\Phi_{2}}-1\right)|0,2\rangle_{a, b}\right] .
\end{aligned}
$$

Interestingly, for all values $N>1$, intensity-difference measurements fails to capture any phase-shift dependence; this results in a measurement of zero irrespective of the value of $\phi$, making it an unsuitable detection observable for this choice of input state.

A Hermitian operator was introduced by Dowling et al. ${ }^{33 \mid 34}$ of the form $\hat{\Sigma}_{N}=|N, 0\rangle_{a, b}\langle 0, N|+| 0, N\rangle_{a, b}\langle N, 0|$ whose expectation value with respect to the state Eq. $62 .\left\langle\hat{\Sigma}_{N}\right\rangle=\cos N \phi$, depends on the phase $N \phi$ with interference fringes whose oscillation period is $N$ times shorter than that of the single-photon case. The phase uncertainty obtained through the error propagation calculus can be found easily to be

$$
(\Delta \phi)^{2}=\frac{\left\langle\hat{\Sigma}_{N}^{2}\right\rangle-\left\langle\hat{\Sigma_{N}}\right\rangle^{2}}{\left(\partial_{\phi}\left\langle\hat{\Sigma_{N}}\right\rangle\right)^{2}}=\frac{1-\cos ^{2} N \phi}{N^{2} \sin ^{2} N \phi}=\frac{1}{N^{2}},
$$

which is an improvement over the classical (SQL) limit by a factor of $1 / \sqrt{N}$. The result of Eq. 66 follows from the heuristic number-phase relation $\Delta \phi \Delta N \geq 1$. For the $N 00 N$ state of Eq. 62, the uncertainty in photon number is $N$, the total average photon number, immediately giving the equality $\Delta \phi=1 / N$. Extrapolating for arbitrary states, we define the Heisenberg limit (HL) $\Delta \phi_{\mathrm{HL}}=1 / \bar{n}_{\text {tot }}$ where $\bar{n}_{\text {tot }}$ is the total average photon number inside the interferometer.

It was found that the results of the projection operator employed by Dowling et al. can be realized through implementation of photon-number parity measurements performed on one mode 24 . As a demonstration, consider the $N=2$ case described by the output state given by Eq. 65 . The action of the parity operator on the $b$-mode, $\hat{\Pi}_{b}=(-1)^{b^{\dagger}} \hat{b}$, results in a sign flip on the center term

$$
\begin{aligned}
& \left.\hat{\Pi}_{b} \mid \text { out }, 2\right\rangle=\frac{1}{2 \sqrt{2}}\left[\left(1-e^{i 2 \phi+\Phi_{2}}\right)|2,0\rangle_{a, b}+\right. \\
& \left.-\sqrt{2} i\left(1+e^{i 2 \phi+\Phi_{2}}\right)|1,1\rangle_{a, b}+\left(e^{i 2 \phi+\Phi_{2}}-1\right)|0,2\rangle_{a, b}\right]
\end{aligned}
$$

leading to the expectation value $\left\langle\hat{\Pi}_{b}\right\rangle=\cos \left(2 \phi+\Phi_{2}\right)$, similar to the result obtained through use of the projection operator employed by Dowling et al. Unlike the method of measuring the intensity-difference between modes, this carries relevant 
phase information from which an estimation can be made. For the arbitrary $N$ case, it was found

$$
\begin{aligned}
\left\langle\hat{\Pi}_{b}\right\rangle & =\frac{i^{N}}{2}\left[e^{i\left(N \phi+\Phi_{N}\right)}+(-1)^{N} e^{-i\left(N \phi+\Phi_{N}\right)}\right] \\
& = \begin{cases}(-1)^{N / 2} \cos \left(N \phi+\Phi_{N}\right) & N \text { even }, \\
(-1)^{(N+1) / 2} \sin \left(N \phi+\Phi_{N}\right) & N \text { odd }\end{cases}
\end{aligned}
$$

and consequently $\Delta \phi=1 / N$. It is important to note that while photon-number parity functions similarly to the projection operator put forth by Dowling et al., they are not equivalent. This is clear as $\hat{\Sigma}_{N}$ is not directly connected to an observable. Furthermore, there is presently no physical realization of the projector $\hat{\Sigma}_{N}$ capable of being utilized experimentally.

Clearly states similar in form to Eq. 62 after the first beam splitter are favorable in interferometry. Let us begin by considering a case in which the state after beam splitting can be written in terms of a superposition of $N 00 N$ states.

\section{B. Entangled coherent states}

One such case known to yield Heisenberg-limited phase sensitivity is the case in which the action of the first beam splitter results in an entangled coherent state $(\mathrm{ECS}) \sqrt{36}$. It has been shown by Israel et al. ${ }^{37}$ that such a state can be generated through the mixing of coherent light with a squeezed vacuum at the first beam splitter (a case to be discussed in greater detail in a latter section). Another method involves the mixing of coherent- and cat- states. The coherent state is given by $|\alpha\rangle=e^{-|\alpha|^{2} / 2} \sum_{n=0}^{\infty} \frac{\alpha^{n}}{\sqrt{n !}}|n\rangle$ and constitutes the most classical of quantized fields states, characterized as light from a well phase-stabilized laser. It is important to point out that while coherent light maintains classical properties, it is still a quantum state of light as it is defined in terms of a quantized electromagnetic field. The generalized cat state $\frac{}{38}$ is expressed as a superposition of equal-amplitude coherent states differing by a $\pi$-phase shift. Such states have been studied extensively in the context of phase-shift measurements 39 . The initial state can be written as

$$
\mid \text { in }\rangle=|\beta\rangle_{a} \otimes \mathscr{N}\left(|\gamma\rangle_{b}+e^{i \theta}|-\gamma\rangle_{b}\right)
$$

where $\mathscr{N}$ is the cat state normalization factor given by $\mathscr{N}=$ $1 / \sqrt{2\left(1+e^{-2|\gamma|^{2}} \cos \theta\right)}$. The QFI can be calculated immediately for this input state using Eq. 54. For large $\gamma,\left\langle\hat{J_{+}}\right\rangle=$ $\left\langle\hat{J_{-}}\right\rangle=\left\langle\hat{J}_{y}\right\rangle \simeq 0$ and

$$
\begin{aligned}
\left\langle J_{+}^{2}\right\rangle & =\beta^{* 2} \gamma^{2}, & \left\langle J_{-}^{2}\right\rangle & =\beta^{2} \gamma^{* 2} \\
\left\langle J_{+} \hat{J}_{-}\right\rangle & =|\beta|^{2}\left(1+|\gamma|^{2}\right), & \left\langle J_{-} \hat{J}_{+}\right\rangle & =|\gamma|^{2}\left(1+|\beta|^{2}\right)
\end{aligned}
$$

resulting in

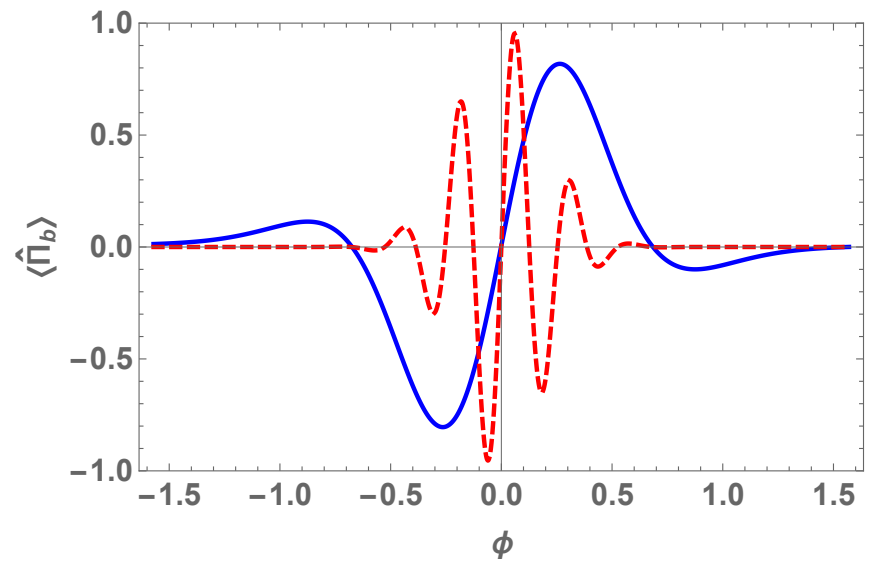

FIG. 4. Expectation value of the parity operator $\left\langle\hat{\Pi}_{b}\right\rangle$ for $\bar{n}_{\text {tot }}=5$ (blue, solid) and $\bar{n}_{\text {tot }}=25$ (red, dashed). The peaks of the curves narrows and migrate towards the phase origin, which serves as an inflection point. Note for these curves, the relative phase shift in Eq. 69 is chosen as $\theta=\pi / 2$ given our interferometric scheme of Eq. 55 to align with the results found by Gerry et a ${ }^{40}$.

$$
\begin{aligned}
F_{Q} & =4\left\langle\left(\Delta \hat{J_{y}}\right)^{2}\right\rangle_{\text {in }}=4\left\langle\hat{J}_{y}^{2}\right\rangle \\
& =|\beta|^{2}+|\gamma|^{2}+2|\beta|^{2}|\gamma|^{2}\left(1-\cos \left[2\left(\theta_{\beta}-\theta_{\gamma}\right)\right]\right),
\end{aligned}
$$

where $\left(\theta_{\beta}-\theta_{\gamma}\right)$ is the phase difference between coherent states $|\beta\rangle$ and $|\gamma\rangle$. Setting $\beta=\alpha / \sqrt{2}$ and $\gamma=-i \beta=$ $-i \alpha / \sqrt{2}$, the total average photon number in the interferometer in the limit of large coherent state amplitude is $|\beta|^{2}+$ $|\gamma|^{2}=|\alpha|^{2}$. Plugging these into Eq. 72 and noting now that $\theta_{\beta}-\theta_{\gamma}=\pi / 2$, the minimum phase uncertainty is given by

$$
\Delta \phi_{\min } \stackrel{\text { large } \alpha}{\longrightarrow} \frac{1}{|\alpha|^{2}}=\Delta \phi_{\mathrm{HL}} .
$$

Let us calculate the state after the first beam splitter for this choice of input state. Through the usual beam splitter transformations for coherent states, we have

$$
\begin{gathered}
|\beta\rangle_{a}|\gamma\rangle_{b} \stackrel{\mathrm{BS} 1}{\longrightarrow}\left|\frac{1}{\sqrt{2}}(\beta+i \gamma)\right\rangle_{a}\left|\frac{1}{\sqrt{2}}(\gamma+i \beta)\right\rangle_{b}, \\
|\beta\rangle_{a}|-\gamma\rangle_{b} \stackrel{\mathrm{BS} 1}{\longrightarrow}\left|\frac{1}{\sqrt{2}}(\beta-i \gamma)\right\rangle_{a}\left|\frac{1}{\sqrt{2}}(-\gamma+i \beta)\right\rangle_{b},
\end{gathered}
$$

making the state after the first beam splitter

$$
\begin{aligned}
\mid \text { out, BS 1 } 1\rangle=\mathscr{N} & \left(\left|\frac{1}{\sqrt{2}}(\beta+i \gamma)\right\rangle_{a}\left|\frac{1}{\sqrt{2}}(\gamma+i \beta)\right\rangle_{b}+\right. \\
& \left.+e^{i \theta}\left|\frac{1}{\sqrt{2}}(\beta-i \gamma)\right\rangle_{a}\left|\frac{1}{\sqrt{2}}(-\gamma+i \beta)\right\rangle_{b}\right] .
\end{aligned}
$$

Once again setting $\beta=\alpha / \sqrt{2}$ and $\gamma=-i \beta=-i \alpha / \sqrt{2}$, this becomes 


$$
\mid \text { out, BS } 1\rangle=\mathscr{N}\left(|\alpha\rangle_{a}|0\rangle_{b}+e^{i \theta}|0\rangle_{a}|i \alpha\rangle_{b}\right)
$$

the entangled coherent state. This state was studied by Gerry et al. ${ }^{40}$ where the first beam splitter was replaced by an asymmetric non-linear interferometer (ANLI) yielding the state $\mid$ out, ANLI $\rangle \propto\left(|0, i \alpha\rangle_{a, b}+i|-\alpha, 0\rangle_{a, b}\right)$, similar to Eq. 76 . This state can be written as a superposition of N00N states as per

$$
\begin{aligned}
\mid \text { out, ANLI }\rangle=e^{-\frac{1}{2}|\alpha|^{2}} \sum_{N=}^{\infty} & \frac{\alpha^{N}}{\sqrt{N !}}(-1)^{N} \times \\
& \times \frac{1}{\sqrt{2}}\left(|N, 0\rangle_{a, b}+e^{-i \Phi_{N}}|0, N\rangle_{a, b}\right),
\end{aligned}
$$

where $\Phi_{N}=\frac{\pi}{2}(N+1)$. Parity-based detection was considered on the output $b$-mode after acquiring a phase shift $\phi$ and passing through the second beam splitter to find

$$
\begin{aligned}
\langle\hat{\Pi}\rangle_{b} & =e^{-\bar{n}}\left(1+e^{\bar{n} \cos \phi} \sin (\bar{n} \sin \phi)\right), \\
\partial_{\phi}\langle\hat{\Pi}\rangle_{b} & =\bar{n} e^{-\bar{n}(1-\cos \phi)} \cos (\phi+\bar{n} \sin \phi),
\end{aligned}
$$

and from the error propagation calculus, Eq. 60, the phase uncertainty for $\phi \rightarrow 0$ is

$$
\Delta \phi_{\hat{\Pi}_{b}}=\frac{\sqrt{1-e^{-2 \bar{n}}}}{\bar{n}},
$$

which subsequently yields the HL in the limit of large $|\alpha|$. Plots of the expectation value of the parity operator, Eq. 78 . and corresponding phase uncertainty, Eq. 80 , found by Gerry et $a l .40$ are given in Figs. 4 and 5 , respectively. Clearly distributions after the first beam splitter that are reminiscent in form to the $N 00 N$ state tends towards providing greater phase sensitivity.

Another such case to consider is the case of the input twinFock states. As per the very well known Hong-Ou-Mandel (HOM) effect, when the initial state $|1,1\rangle_{a, b}$ is incident upon a 50:50 beam splitter, the resulting state is the two-photon $N 00 N$ state $|2,0\rangle_{a, b}+|0,2\rangle_{a, b}$. Let us now consider the case in which the arbitrary state $|N, N\rangle_{a, b}$ is taken as the initial state.

\section{Twin-Fock state input}

It was first pointed out by Holland and Burnett ${ }^{41}$ that interferometric phase measurements when considering input twinFock states $|N, N\rangle_{a, b}$ asymptotically approaches the HL. They found this by studying the phase-difference distribution for the states inside the MZI just prior to the second beam splitter. On the other hand, Bollinger et al. ${ }^{7 \mid+2}$ showed that if the state just

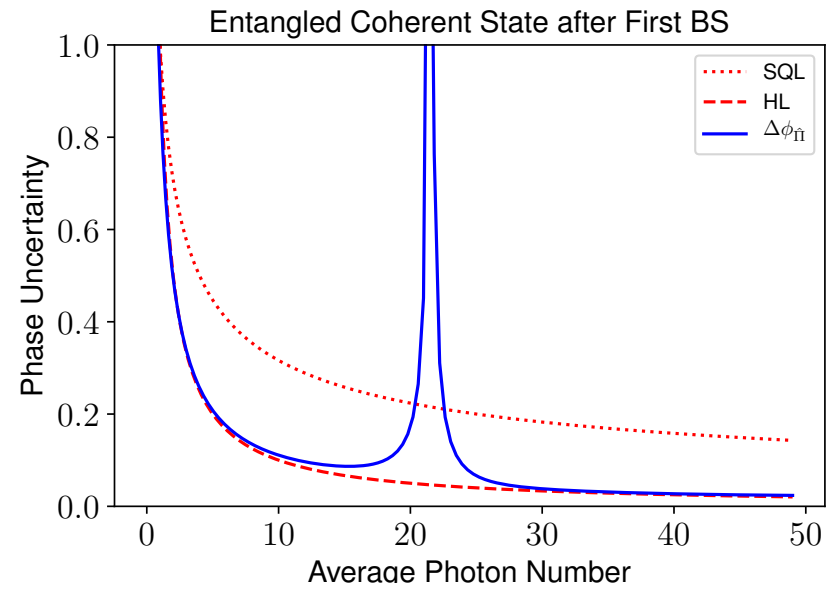

FIG. 5. Phase uncertainty obtained when the state after the first beam splitter is an entangled coherent state of the form Eq.76 for a relative phase $\theta=\pi / 2$ and phase value $\phi=\pi / 45$. Note that aside from the spike at $\bar{n}_{\text {tot }} \sim 21$, the phase uncertainty follows closely along the HL.

after the first beam splitter is somehow a maximally entangled state (MES) of the form $\propto|2 N, 0\rangle_{a, b}+e^{-i \Phi_{N}}|0,2 N\rangle_{a, b}$, $N \in \mathbb{Z}^{+}$, then the phase uncertainty is exactly the HL: $\Delta \phi_{\mathrm{HL}}=$ $1 / 2 N$. The problem is such a state is incredibly difficult to produce; in fact, it cannot be done with an ordinary beam splitter. Schemes for generating such states using both nonlinear devices and linear devices used in conjunction with conditional measurements have been proposed 23|| $43|44| 45|| 46|+7| \mid 48$. For an initial state described by state coefficients $C_{n}^{(1)} C_{q}^{(2)}=$ $\delta_{N, n} \delta_{N, q}$ as per Eq. 56, the state after the first beam splitter is

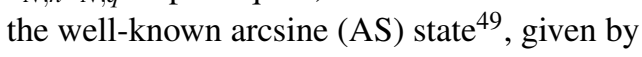

$$
\begin{aligned}
\mid \text { out, BS1 }\rangle & =\sum_{k=0}^{N}(-1)^{N-k}\left[\left(\begin{array}{c}
2 k \\
k
\end{array}\right)\left(\begin{array}{c}
2 N-2 k \\
N-k
\end{array}\right)\left(\frac{1}{2}\right)^{2 N}\right]^{1 / 2} \times, \\
& \times|2 k\rangle_{a} \otimes|2 N-2 k\rangle_{b},
\end{aligned}
$$

where in this case a $\hat{J}_{y}$-type beam splitter was considered rather than a $\hat{J}_{x}$-type of the previous sections. This simply amounts to a relative phase difference between terms in the sum in Eq. 81. Clear for the $N=1$ case, we recover the well known two-photon $N 00 N$ state that has long been available in the laboratory 50 . For $N>1$, the state of Eq. 81 does not cleanly result in the $N$-photon $N 00 N$ state, but instead a superposition of the $N$-photon $N 00 N$ state and other (but not all) permutations of the state $|p, q\rangle$ where $p+q=2 N$. Due to the strong correlations between photon number states of the two modes, the only nonzero elements of the joint-photon number probability distribution are the joint probabilities for finding $2 k$ photons in the $a$-mode and $2 N-2 k$ photons in the $b$-mode, given by 


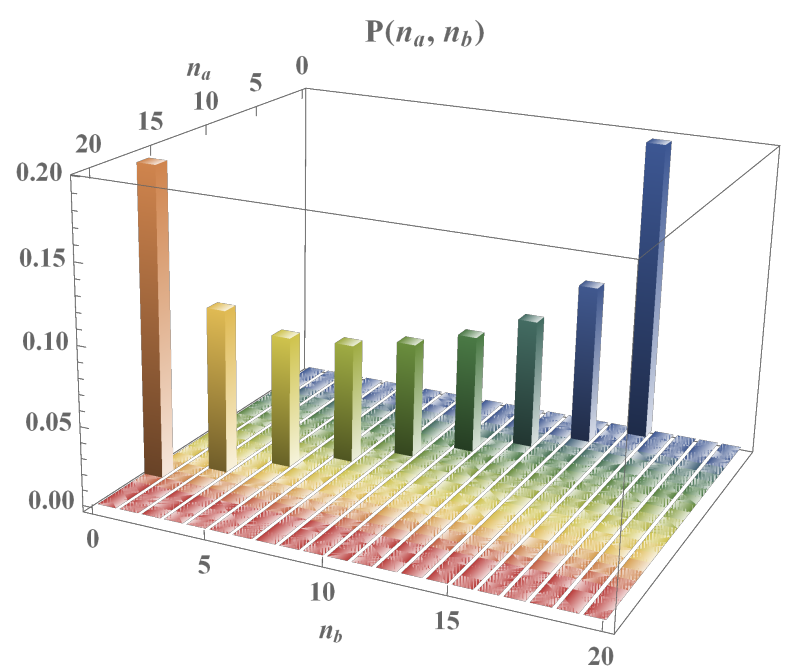

FIG. 6. Two-mode joint photon number distribution resulting from beamsplitting the state $\mid$ in $\rangle=|N, N\rangle_{a, b}$. The "bathtub" shape of the arcsine distribution is peaked at the $|N, 0\rangle_{a, b}$ and $|0, N\rangle_{a, b}$ states. This is plotted for $N=8$.

$$
\begin{aligned}
P_{\mathrm{AS}}(2 k, 2 N-2 k) & \left.=\left.\right|_{a, b}\langle 2 k, 2 N-2 k| \text { out, BS } 1\right\rangle\left.\right|^{2} \\
& =\left(\begin{array}{c}
2 k \\
k
\end{array}\right)\left(\begin{array}{c}
2 N-2 k \\
N-k
\end{array}\right)\left(\frac{1}{2}\right)^{2 N}, \quad k \in[0, N]
\end{aligned}
$$

forming a distribution known as the fixed-multiplicative discrete arcsine law of order $N \underline{51}$, deriving the name of the state. Such a distribution is characterized by the "bathtub" shape of an arcsine distribution, with peaks occuring for the $|N, 0\rangle_{a, b}$ and $|0, N\rangle_{a, b}$ states, as shown in Fig. 6. The phase properties of this state were studied by Campos et al. ${ }^{49}$.

Next we will compare the qCRB for this choice of initial state against the phase uncertainty obtained via parity-based measurements. Once again, the qCRB can be calculated directly from the initial state through Eq. 54 It is plainly evident that $\langle J\rangle_{+}=\langle J\rangle_{-}=\langle J\rangle_{y}=0,\left\langle\hat{J}_{+} \hat{J}_{-}\right\rangle=\left\langle\hat{J}_{-} \hat{J}_{+}\right\rangle=N(N+1)$ and consequently

$$
\Delta^{2} \hat{J}_{y}=\frac{N}{2}(1+N) \rightarrow \Delta \phi_{\min }=\frac{1}{\sqrt{2 N(1+N)}}
$$

Right away it is clear that for the case of $N=1$, the minimum phase uncertainty provides the HL: $\Delta \phi_{\mathrm{HL}}=1 / 2 N=1 / 2$. Next we consider the use of parity performed on the output $b$-mode as a detection observable. The expectation value of the parity operator can be calculated directly with respect to the state of Eq. 81, accounting for the phase shift and assuming the second beam splitter is of the $\hat{J}_{x}$-type, to be

$$
\begin{aligned}
\left\langle\hat{\Pi}_{b}\right\rangle_{\mathrm{AS}} & =\left\langle\text { in }\left|\hat{\Pi}_{b}\right| \text { in }\right\rangle \\
& =\left\langle\text { out, BS } 1\left|e^{-i \phi \hat{J}_{z}} e^{-i \frac{\pi}{2} \hat{J}_{x}} \hat{\Pi}_{b} e^{i \frac{\pi}{2} \hat{J}_{x}} e^{i \phi \hat{J}_{z}}\right| \text { out, BS } 1\right\rangle \\
& =\sum_{k=0}^{N} e^{i 2 \phi(N-2 k)}\left(\begin{array}{c}
2 k \\
k
\end{array}\right)\left(\begin{array}{c}
2 N-2 k \\
N-k
\end{array}\right)\left(\frac{1}{2}\right)^{2 N},
\end{aligned}
$$

The imaginary part of Eq. 84 sums identically to zero as it is the product of an even times and odd function of $k$. The real part is identically a Legendre polynomial, making

$$
\left\langle\hat{\Pi}_{b}\right\rangle_{\mathrm{AS}}=P_{N}[\cos (2 \phi)] .
$$

With this, the phase uncertainty can be computed directly from the error propagation calculus given by Eq. 60. For $N=1$, the expectation value of the parity operator is $\left\langle\hat{\Pi}_{b}\right\rangle_{\mathrm{AS}}=\cos (2 \phi)$ leading to $\Delta \phi=1 / 2$, the HL. For $N=2$, it can be shown $\left\langle\hat{\Pi}_{b}\right\rangle_{\mathrm{AS}}=1 / 4+3 / 4 \cos (4 \phi)$, which in the limit of $\phi \rightarrow 0$ yields $\Delta \phi=1 / \sqrt{12}=0.2886$; close to the HL of $1 / 4=0.25$. These results are in agreement with the minimum phase uncertainty obtained through calculation of the qCRB for this state, Eq. 83. A plot of the parity-based phase uncertainty and corresponding $\mathrm{qCRB}$ can be found in Fig. 7. Another interesting feature of Eq. 85 occurs when considering measurements around $\phi=\pi / 2$. Through use of standard identities involving Legendre polynomials, it can be shown that Eq. 85 becomes $\left\langle\hat{\Pi}_{b}\right\rangle_{\mathrm{AS}}^{(\phi=\pi / 2)}=(-1)^{N}$, corresponding to a peak in the curve yielding the same degree of resolution as $\phi=0$, but takes the maximal (minimal) value of \pm 1 depending on the value of $N$. This may prove to be of use in verifying one has lossless conditions within the interferometer as typically the experimenter would have foreknowledge of the value $N$ and therefore know what the value in a $\pi / 2$-shifted interferometer should be. Measurement to the contrary can point towards the presence of losses in the system.

In the next section, we will briefly consider states comprised of superpositions of twin-Fock states.

\section{Superpositions of twin-Fock states}

Highly photon-number correlated continuous variable twomode states have been investigated for use in quantum optical interferometry. It is immediately clear that such states are entangled as they are already in Schmidt form $|\Psi\rangle=$ $\sum_{n=0}^{\infty} B_{n}|n, n\rangle_{a, b}$ with total average photon number $\bar{n}_{\text {tot }}=$ $2 \sum_{n=0}^{\infty}\left|B_{n}\right|^{2} n$. In terms of the initial state of Eq. 56 the coefficients for such a state are given by $C_{n}^{(1)} C_{q}^{(2)}=B_{n} \delta_{q, n}$. The expectation value of the parity operator is readily calculable for arbitrary correlated two-mode states of this form using the results of the twin-Fock state input of Eq. 85 .

$$
\left\langle\hat{\Pi}_{b}\right\rangle_{\text {corr }}=\sum_{n=0}^{\infty}\left|B_{n}\right|^{2} P_{n}[\cos (2 \phi)]
$$




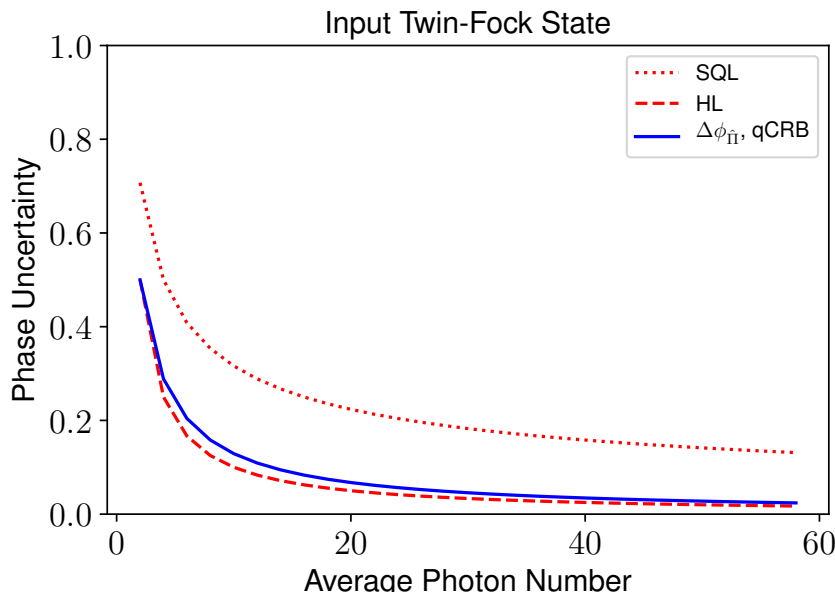

FIG. 7. Parity-based phase uncertainty with phase $\phi=10^{-4}$ for the case of an input twin-Fock state. Note the phase uncertainty obtained corresponds with the qCRB.

where once again $P_{n}(x)$ are the Legendre polynomial. The most well-known and studied correlated two-mode state is the two-mode squeezed vacuum state (TMSVS). The TMSVS is a laboratory standard, routinely produced through parametric down conversion ${ }^{52}$ : a second order nonlinear effect in which a pump photon of frequency $2 \omega$ is annihilated and two photons, each of frequency $\omega$, are produced. The correlation between modes is due to the pair-creation of photons resulting from the down-conversion process. Note that under the parametric approximation, the pump is treated as a classical and non-depleting field. Consequently the pump is not treated as a quantized mode. The TMSVS state coefficients are given by $B_{n}^{(\text {TMSVS })}=\left(1-|z|^{2}\right)^{1 / 2} z^{n}$. The parameter $z$ is a complex number constrained to be $|z|<1$ and can be expressed in terms of the pump field parameters, $\gamma$ and $2 \phi$ being the pump amplitude and phase respectively, as $z=e^{i 2 \phi} \tanh r$ where $r=|\gamma| t$ is the squeeze parameter. Interestingly, coupling the TMSVS coefficients with Eq. 86 yields for the phase value $\phi=\pi / 2$

$$
\left\langle\hat{\Pi}_{b}\right\rangle_{\mathrm{tmsvs}}^{(\phi=\pi / 2)}=\frac{1}{\cosh (2 r)}= \begin{cases}1, & r \rightarrow 0 \\ 2 e^{-2 r} . & r \gg 1\end{cases}
$$

In other words, measurement of photon-number parity on one of the output modes provides a direct measure of the degree of squeezing i.e., determination of the squeeze parameter $r$. For the TMSVS, parity-based detection yields a phase uncertainty that falls below the HL for $\phi=0$; an effect that has been pointed out in the past by Anisimov et al. [31, explained in terms of the Fisher information. Once again using Eq. 54 and noting for such a correlated state that $F_{Q}=2\left\langle\hat{J}_{+} \hat{J}_{-}\right\rangle_{\text {in }}$, it can be shown that the TMSVS minimum phase uncertainty is

$$
\Delta \phi_{\min }=\frac{1}{\sqrt{\bar{n}_{\text {tot }}\left(2+\bar{n}_{\text {tot }}\right)}},
$$

where for the TMSVS $\bar{n}_{\mathrm{tot}}=2 \sinh ^{2} r$. This means that the
TMSVS has the potential for super sensitive phase estimation; clearly the phase estimate of Eq. 88 is sub-HL. This is seemingly a violation of the bound set for quantum states. It has been argued that such a limitation, based on the heuristic photon number-phase relation $\Delta \phi \Delta N \geq 1$, is reasonable in the case of definite photon number (finite energy) but proves to be an incomplete analysis when considering the effect of photon-number fluctuations. Hoffmar ${ }^{25}$ suggests a more direct definition of the limit on phase estimation for quantum states in terms of the second moment of $\hat{n}, \Delta \varphi=1 /\left\langle\hat{n}^{2}\right\rangle$. This provides better sensitivity in phase measurements than the HL as $\left\langle\hat{n}^{2}\right\rangle$ contains direct information about fluctuations that $\langle\hat{n}\rangle^{2}$ does not. In fact this is why parity-based measurement yields greater sensitivity: it contains all moments of $\hat{n}$. For the case of the TMSVS, the Hoffman limit is given by $\Delta \phi=1 / \sqrt{2 \bar{n}(\bar{n}+1)}$. With parity-based detection, sensitivity of the phase estimate is better than allowed by the HL but is never better than the Hoffman limit (the sub-HL sensitivity is prominent for low (but still $>1$ ) average photon numbers but asymptotically converges to the HL for $\phi=0$ ). It was pointed out by Anisimov et al..$^{31}$ as well as Gerry et al. ${ }^{[33}$ that the TMSVS, using parity measurements, has superior phase sensitivity near $\phi=0$ but degrades rapidly as the phase difference deviates from zero as shown in Fig. 8, making the state sub-optimal for interferometry. On the other hand, the case in which one has parametric down-conversion with coherent states seeding the signal and idler modes, or twomode squeezed coherent states (TMSCS), was considered by Birrittella et al ${ }^{[54}$ who found a measurement resolution and phase sensitivity dependent on a so-called cumulative phase (the sum of the initial field phases) that yields, for low squeezing, sub-SQL phase sensitivity that does not degrade like the TMSVS does as the phase deviates from zero for the optimal choice of cumulative phase. The cumulative-phasedependent state statistics and entanglement properties of the state resulting from coherently-stimulated down-conversion with a quantized pump field has been studied by Birrittella et $a l^{[5]}$, however the state has not yet been considered in the context of interferometry.

Another correlated two-mode state that has been extensively studied in the context of interferometry and metrology are the pair coherent states $(\mathrm{PCS}) \sqrt{56}$, or circle states, which have the form

$$
|\zeta\rangle=\mathscr{N} \int_{0}^{2 \pi}\left|\sqrt{\zeta} e^{i \theta}\right\rangle\left|\sqrt{\zeta} e^{-i \theta}\right\rangle d \theta
$$

where $\left|\sqrt{\zeta} e^{ \pm i \theta}\right\rangle$ are the Glauber coherent states. In terms of the number state basis, the PCS can be written as

$$
|\zeta\rangle=\frac{1}{\sqrt{I_{0}(2|\zeta|)}} \sum_{n=0}^{\infty} \frac{\zeta^{n}}{n !}|n, n\rangle_{a, b},
$$

where $I_{0}(2|\zeta|)$ is the modified Bessel function of order zero and $\zeta$ is a complex number defined such that $|\zeta\rangle$ is a righteigenstate of the joint photon-annihilation operators $\hat{a} \hat{b}|\zeta\rangle=$ 


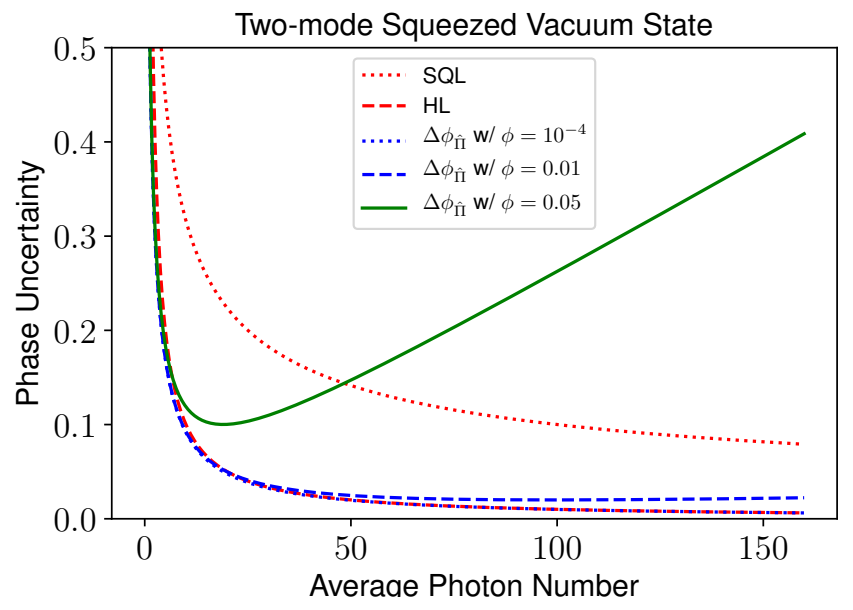

FIG. 8. Phase uncertainty using parity-based detection for the input two-mode squeezed vacuum state. For phases close to zero, the phase uncertainty yields sub-HL sensitivity, however rapidly degrades as the phase deviates from zero.

$\zeta|\zeta\rangle$ and $\left(\hat{a}^{\dagger} \hat{a}-\hat{b}^{\dagger} \hat{b}\right)|\zeta\rangle=0 \frac{57}{\text {. Such a state has been shown }}$ to exhibit sub-Poissonian statistics which results in sub-SQL phase uncertainty, enhanced measurement resolution and a high signal-to-noise ratio: results very close to those obtained from input twin-Fock states ${ }^{53}$. Furthermore, the PCS does not display the degradation of phase sensitivity as $\phi$ deviates from zero as the TMSVS does, making it more stable for interferometric measurements. The problem, however, lies in generating such a state. Several schemes have been proposed, most notably a scheme involving the use of third order cross-Kerr coupling between coherent states and the implementation of a state-reductive measuremen $[58$, resulting in the projection of the PCS in bursts. Currently, the PCS has yet to be experimentally realized.

\section{Coherent light mixed with a squeezed vacuum state}

The original interferometric scheme for reducing measurement error was proposed by Caves ${ }^{59}$ in the context of gravitational wave detection. Here we consider the case in which the input state is a product of coherent light in one port and a single-mode squeezed vacuum state (SVS) in the other, given by $|\mathrm{SVS}\rangle=\sum_{N=0}^{\infty} S_{N}|N\rangle$ with average photon number $\bar{n}_{\text {SVS }}=\sinh ^{2} r$, and with state coefficients

$$
S_{N}=(-1)^{N / 2}\left[\frac{N !}{2^{N}\left(\frac{N}{2} !\right)^{2}} \frac{\tanh ^{N} r}{\cosh r}\right]^{1 / 2} \cos ^{2}(N \pi / 2),
$$

where once again the parameter $r$ is the squeeze parameter. Such a state can be generated by beam splitting a TMSVS; the resulting two-mode state is a product of two single-mode SVS offset from each other by a $\pi$-phase shift ${ }^{60}$. The initial state is then $\mid$ in $\rangle=|\alpha\rangle_{a} \otimes|\mathrm{SVS}\rangle_{b}$ with state coefficients as per Eq. 56 given by $C_{n}^{(1)} C_{p}^{(2)}=e^{-|\alpha|^{2} / 2} \frac{\alpha^{n}}{\sqrt{N !}} \times S_{p}$. This choice of input state has been shown to produce the ECS after the first beam splitter ${ }^{37}$ and has been studied extensively by Pezzé et al. ${ }^{61}$ who considered a Bayesian phase inference protocol and showed that the phase sensitivity saturates the CRB and go on to demonstrate that the phase sensitivity can reach the HL $\Delta \phi \sim 1 / \bar{n}_{\text {tot }}$ independently of the true value of the phase shift. As the input state is path-symmetric ${ }^{26}$, it is sufficient to determine the CRB through calculation of the classical Fisher information, which they found for a single measurement to be

$$
\begin{aligned}
F^{(1)}(\phi) & =\sum_{N_{c}, N_{d}}^{\infty} \frac{1}{P\left(N_{c}, N_{d} \mid \phi\right)}\left(\frac{\partial P\left(N_{c}, N_{d} \mid \phi\right)}{\partial \phi}\right)^{2} \\
& =|\alpha|^{2} e^{2 r}+\sinh ^{2} r
\end{aligned}
$$

where $P\left(N_{c}, N_{d} \mid \phi\right)$ is the conditional probability of measuring $N_{c}$ photons in one output mode and $N_{d}$ in the other and is given in terms of the Wigner- $d$ rotation elements (see Appendix B) by

$$
P\left(N_{c}, N_{d} \mid \phi\right)=e^{-|\alpha|^{2}}\left|\sum_{n=0}^{2 j} \frac{\alpha^{2 j-n}}{(2 j-n) !} S_{n} d_{m, j-n}^{j}(\phi)\right|^{2},
$$

with $j=\left(N_{c}+N_{d}\right) / 2$ and $m=\left(N_{c}-N_{d}\right) / 2$. For the regime in which both input ports are of roughly equal intensity $|\alpha|^{2} \simeq$ $\sinh ^{2} r=\bar{n}_{\text {tot }} / 2$,and assuming large $r$ such that $\bar{n}_{\text {tot }} \simeq e^{2 r} / 2$ it can be shown

$$
\Delta \phi_{\min }=\frac{1}{\sqrt{\bar{n}_{\mathrm{tot}}\left(\bar{n}_{\mathrm{tot}}+\frac{1}{2}\right)}} \stackrel{\text { large } \bar{n}_{\mathrm{tot}}}{\longrightarrow} \frac{1}{\bar{n}_{\mathrm{tot}}}=\Delta \phi_{\mathrm{HL}} .
$$

The same scheme was considered by Birrittella et al ${ }^{[62}$ using photon-number parity performed in the output $b$-mode as the detection observable. In particular, they studied a regime in which the two-mode joint-photon number distribution was parameterized such that the it was symmetrically populated along the borders with no population in the interior, mimicking the case of the $N 00 N$ state generated after the first beam splitter. The parameters $\alpha$ and $r$ were chosen to be relevant to an experiment performed by Afek et al ${ }^{[63}$ based on the $N 00 N$ state within the superposition of $N 00 N$ states (found in the output state of the first beam splitter) in which they obtained high phase sensitivity and super-resolution. They achieved this measurement scheme by counting only the coincident counts where the total photon numbers counted added up to the selected value of $N$. In other words, they measured $\left\langle\hat{a}^{\dagger} \hat{a} \hat{b}^{\dagger} \hat{b}\right\rangle_{\text {out }}$ but retained only the counts where if one detector detects $m$ photons, the other detects $N-m$, and where all other counts $\neq N$ are discarded. They reported sub-SQL phase sensitivity with this scheme as well as super-resolved measurement. It is worth pointing out, however, that this tends to work better for low average photon numbers as the large-photon-number case cannot be reasonably expressed as a superposition of $N 00 N$ states. 
With parity measurements performed on one of the output beams, it is not necessary nor possible to restrict oneself to a definite $N$-photon $N 00 N$ state, which can be advantageous. The total number of photons inside the interferometer for this input state is indeterminate, but the Heisenberg limit is approached in terms of the average of the total photon number. It has been shown by Seshadreesan et al. ${ }^{64}$ that photon-number parity-based interferometry reaches the HL in the case of equal-intensity light incident upon a 50:50 beam splitter. The use of photon-subtracted squeezed light in one of the input ports has also been studied by Birrittella et al. $\frac{65}{}$ who showed the state after the first beam splitter resembled an ECS of higher average photon number than that generated through the use of a squeezed vacuum state.

Next we will consider the case in which purely classical light is initially in one mode and the most quantum of quantized field states, a Fock (or number state), is initially in the other.

\section{E. Coherent light mixed with $N$ photons}

Next we consider the choice of input state $\mid$ in $\rangle=|\alpha\rangle_{a} \otimes|N\rangle_{b}$, that is, coherent light in the input $a$-mode and a Fock state of $N$ photons in the input $b$-mode. This was studied by Birrittella et $a l .^{62}$ in the context of parity-based phase estimation. In terms of Eq. 56, the two-mode state coefficients are $C_{n}^{(1)} C_{q}^{(2)}=e^{-|\alpha|^{2} / 2} \alpha^{n} / \sqrt{n !} \times \delta_{q, N}$, which in terms of the angular momentum basis states can be written as

$$
\mid \text { in }\rangle=e^{-\frac{1}{2}|\alpha|^{2}} \sum_{j=N / 2, . .}^{\infty} \frac{\alpha^{2 j-N}}{\sqrt{(2 j-N) !}}|j, j-N\rangle,
$$

where the sum over $j$ includes all half-odd integers. It is worth pointing out a characteristic of this particular state upon beam splitting. Consider the state after the first beam splitter, given by (see Appendix A 22 |out, BS 1$\rangle=e^{-i \frac{\pi}{2} \hat{J}_{x}} \mid$ in $\rangle$. For the special case of $N=1$, this state can be written as

$$
\begin{aligned}
\mid \text { out, BS } 1\rangle_{N=1}=e^{-\frac{1}{2}|\alpha|^{2}} & \sum_{n=0}^{\infty} \sum_{q=0}^{\infty} \frac{\left(\frac{\alpha}{\sqrt{2}}\right)^{n}\left(\frac{i \alpha}{\sqrt{2}}\right)^{q}}{\sqrt{n ! p !}} \times \\
& \times\left(1-\delta_{n, 0} \delta_{q, 0}\right) \gamma_{n, q}|n, q\rangle_{a, b}
\end{aligned}
$$

where the factor $\gamma_{n, q}$ is given by

$$
\gamma_{n, q}=\frac{4 i(-1)^{1-q} 2^{n+q+1} n !}{\alpha(n+q+1) !}{ }_{2} \tilde{F}_{1}(2, n+1 ; 2-q ;-1),
$$

and where ${ }_{2} \tilde{F}_{1}(a, b ; c ; z)$ is a regularized hypergeometric function (see Appendix B 1). For $n=q$, this function is identically zero $\forall q \neq 0$. Note that the term $n=q=0$ is a term that does not appear in the sum in Eq. 96 (due to the presence of the $N=1$ initial state). This coincides with a line of destructive interference along the diagonal line $n=q$ resulting in a bimodal distribution. This effect persists for odd values of $N$ resulting in a symmetric (assuming 50:50 beam splitter) $(N+1)$-modal distribution with the peaks of the distribution migrating towards the respective axes. The same structure of distribution occurs for even $N$ as well, however lines of contiguous zeroes do not occur. This is worth noting since a distribution like this is reminiscent of the well known twinFock state input case, discussed earlier, in which the state after beam splitting are the so-called arcsine, or "bat", states 49 . It has long been known that the input twin-Fock state case, and states with similar distributions post-beam splitter, leads to sub-SQL sensitivity $24|33| 34 \mid 49$.

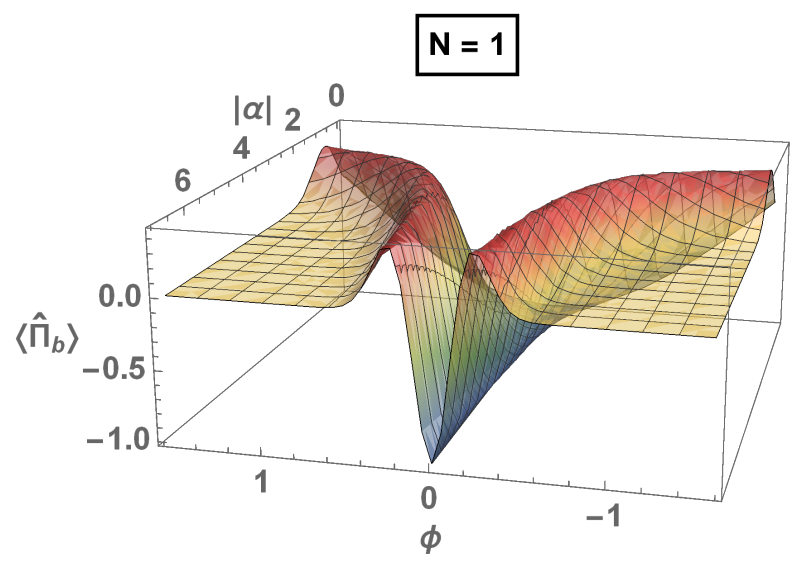

(a)

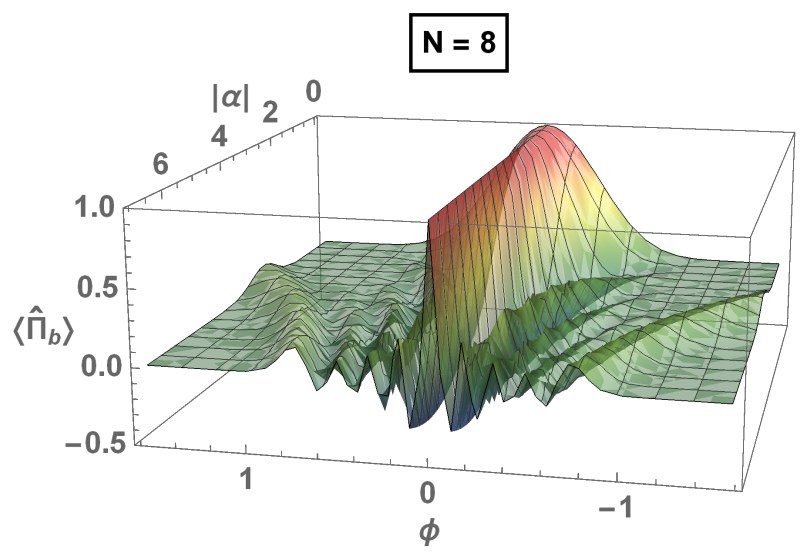

(b)

FIG. 9. Expectation value of the parity operator taken with respect to the output $b$-mode for an input state $\mid$ in $\rangle=|\alpha\rangle_{a}|N\rangle_{b}$ for (a) $N=1$ and (b) $N=8$. Note the narrowness of the peak for increasing $|\alpha|$ and $N$.

\section{Measurement resolution using parity-based detection}

Once again we consider the use of photon-number parity performed on the output $b$-mode as our detection observable. Using in input state of Eq. 95, the expectation value of the parity operator can be computed from Eqs. 58 and 59 to find 


$$
\left\langle\hat{\Pi}_{b}(\phi)\right\rangle_{N}=(-1)^{N} e^{-|\alpha|^{2}} \sum_{j=N / 2, . .}^{\infty} \frac{|\alpha|^{2(2 j-N)}}{(2 j-N) !} d_{j-N, j-N}^{j}(2 \phi) .
$$

In the special case of $N=0$, we obtain the result found by Chiruvelli and Lee ${ }^{66}$ and discussed by Gao et al ${ }^{67}$, which will be expanded upon in greater detail in the following section. For small angles, $\phi \rightarrow 0,\left\langle\hat{\Pi}_{b}(0)\right\rangle_{0} \rightarrow 1$ but becomes narrower about $\phi=0$ for increasing values of $|\alpha|$, as seen in Fig. 9 . The signal is not super-resolved in the usual sense of having oscillation frequencies scaling as $M \phi$ for integer $M>1$. However, compared with the corresponding result for output subtraction, we can see the signal for parity measurement is much narrower, seen in Fig. 11. It is in this sense that Gao et al. ${ }^{[67}$ interpret parity-based measurement to be super-resolved. Furthermore, for arbitrary $N$, the parity of the state is reflected by the expectation value of the parity operator evaluated at $\phi=0$ : $\left\langle\hat{\Pi}_{b}(0)\right\rangle_{N}=(-1)^{N}$. The peak (or trough) centered at $\phi=0$ also narrows for increasing values of $N$. This can also be seen in Fig. 9

\section{Phase uncertainty: approaching the Heisenberg limit}

We begin with an analysis of the phase uncertainty obtained by computation of Eq. 60 with $\hat{J}_{z}$ as the detection observable. Plugging in the coefficients obtained using Eq. 58 for this choice of initial state and using Eq. 59 yields a phase uncertainty

$$
\Delta \phi_{\hat{J}_{z}}=\frac{\sqrt{|\alpha|^{2}+N\left(1+2|\alpha|^{2} \sin ^{2} \phi\right)}}{\left|\left(|\alpha|^{2}-N\right) \sin \phi\right|} .
$$

For $N=0$, we recover the well known SQL. What is important to note about Eq. 99 is that for fixed $|\alpha|$ the optimal noise reduction achievable is the SQL and occurs only for the case of $N=0$. For other values of $N$, the noise level rises to above the SQL. In particular, if the initial average photon numbers of the two modes are around the same value, i.e. $|\alpha|^{2} \simeq N$, the noise level becomes very high.

Parity-based detection fares quite a bit better. The phase uncertainty in this case is computed numerically using Eq. 60 with $\hat{\Pi}_{b}$ as the detection observable. The phase uncertainty, along with corresponding SQL and HL, $\Delta \phi_{\mathrm{SQL}}=1 / \sqrt{|\alpha|^{2}+N}$ and $\Delta \phi_{\mathrm{HL}}=1 /\left(|\alpha|^{2}+N\right)$ respectively, are plotted for several different values of $N$ in Fig. 10 . The largest gain in sensitivity is achieved in the case of going from $N=0$, wherein the phase uncertainty falls along the $\mathrm{SQL}$, to $N=1$, where the phase uncertainty is sub-SQL. The noise reduction approaches the HL for increasing values of $N$.

One can also calculate the QFI using Eq. 54 and subsequent $\mathrm{qCRB}$, which we remind the reader is independent of detection observable and depends solely on the choice of initial state, to find the minimum phase uncertainty attainable ${ }^{62}$

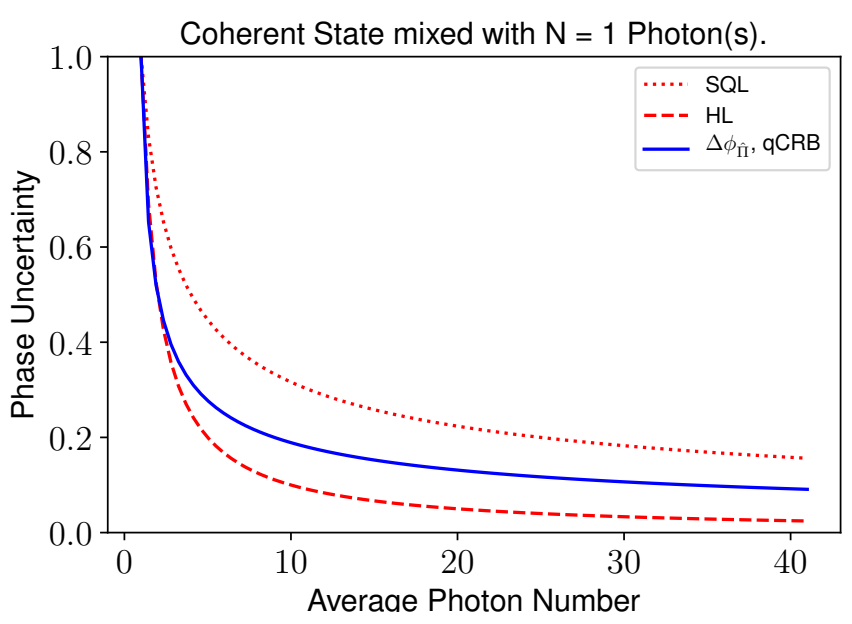

(a)

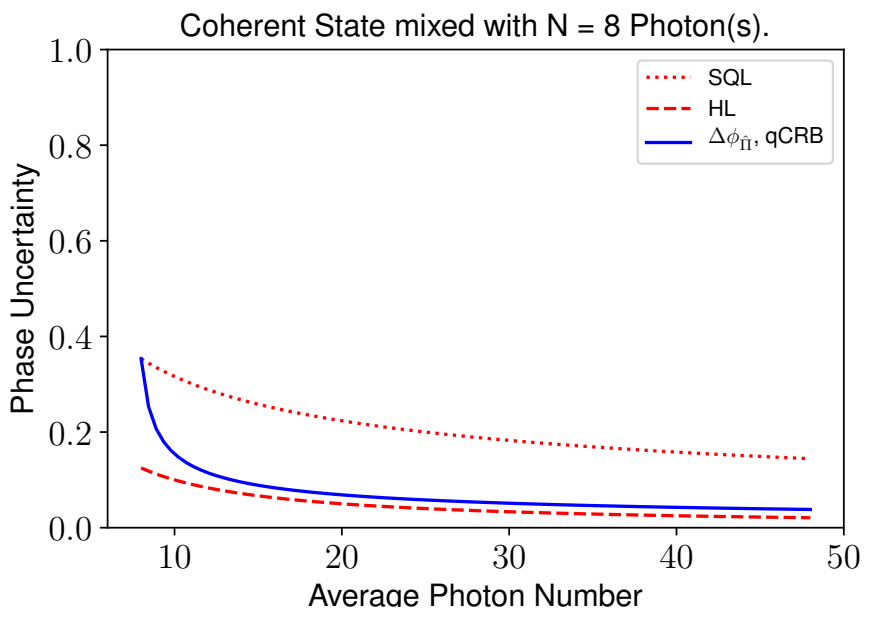

(b)

FIG. 10. Phase uncertainty computed with phase $\phi=10^{-4}$ for the input state $\mid$ in $\rangle=|\alpha\rangle_{a}|N\rangle_{b}$, with accompanying SQL and HL, for(a) $N=1$ and (b) $N=8$. In the limit of $N \rightarrow \infty$, the phase uncertainty approaches the HL. The blue line in each figure also corresponds to the $\mathrm{qCRB}$ for this choice of initial state.

$$
\Delta \phi_{\min }=\frac{1}{\sqrt{|\alpha|^{2}+N\left(1+2|\alpha|^{2}\right)}} .
$$

This result follows the blue curve corresponding to paritybased phase sensitivity in Fig. 10 precisely for small values of the phase. It is worth pointing out that for small $\alpha$, the phase uncertainty scales as $1 / \sqrt{N}$, which follows the SQL.

\section{F. Interferometry with strictly classical (coherent) light}

So far we have investigated the use of several different quantum states of light as our initial state to the MZI and have shown sub-SQL phase sensitivity in all cases when considering parity-based detection. Now we will turn our 
attention to the case where strictly classical light is used. It is well known that this case will yield phase sensitivities at the SQL, the greatest sensitivity attainable using classical light, for the optimal choice of phase when considering intensity-difference measurements. So the question is: what is to be gained by considering the use of photon-number parity? Here we will endeavor to shed some light on this question.

We start by considering coherent light in one of the input ports of the interferometer, making our initial state $\mid$ in $\rangle=$ $|\alpha\rangle_{a} \otimes|0\rangle_{b}$, where once again the coherent state is given by $|\alpha\rangle=e^{-|\alpha|^{2} / 2} \sum_{n=0}^{\infty} \frac{\alpha^{n}}{\sqrt{n !}}|n\rangle$. Considering the transformation of Eq. 55 in which the beam splitters are of $\hat{J}_{x}$-type, the state after the first beam splitter is given by (see Appendix A 3)

$$
|\alpha\rangle_{a}|0\rangle_{b} \stackrel{\mathrm{BS} 1}{\longrightarrow}\left|\frac{\alpha}{\sqrt{2}}\right\rangle_{a}\left|-\frac{i \alpha}{\sqrt{2}}\right\rangle_{b},
$$

and the state after the accumulated phase shift in the $b$-mode (the $\hat{J}_{z}$ operation introduces an anti-symmetric phase shift of $\pm \phi / 2$ in each mode, which can be treated as a phase shift of $\phi$ in one arm of the MZI without loss of generality) is given by

$$
\left|\frac{\alpha}{\sqrt{2}}\right\rangle_{a}\left|\frac{i \alpha}{\sqrt{2}}\right\rangle_{b} \stackrel{\phi \text {-shift }}{\longrightarrow}\left|\frac{\alpha}{\sqrt{2}}\right\rangle_{a}\left|-\frac{i \alpha e^{i \phi}}{\sqrt{2}}\right\rangle_{b} .
$$

Finally, the state after the second beam splitter is

$$
\left|\frac{\alpha}{\sqrt{2}}\right\rangle_{a}\left|-\frac{i \alpha e^{i \phi}}{\sqrt{2}}\right\rangle_{b} \stackrel{\mathrm{BS} 2}{\longrightarrow}\left|\frac{\alpha}{2}\left(1+e^{i \phi}\right)\right\rangle_{a}\left|\frac{i \alpha}{2}\left(1-e^{i \phi}\right)\right\rangle_{b} .
$$

Next we compare the phase uncertainty obtained using two different detection observables: taking the intensitydifference between modes and performing photon number parity on one of the output modes.

\section{Difference in output mode intensities}

The intensity of a quantized field is proportional to the average photon number of the quantum state of the field $\sqrt{68}, I \propto\langle\hat{n}\rangle$. Consequently, the difference between mode intensities at the output of the second beam splitter can be written as $\delta I \propto$ $\left\langle\hat{a}^{\dagger} \hat{a}-\hat{b}^{\dagger} \hat{b}\right\rangle$. In terms of the su(2) Lie algebra, this amounts to the expectation value of the operator $2 \hat{J}_{z}$. Using Eq. 103 , the mode intensities are given given by $\left\langle\hat{n}_{a(b)}\right\rangle=\frac{\bar{n}_{0}}{2}(1 \pm \cos \phi)$ where $\bar{n}_{0}=|\alpha|^{2}$ (note we are assuming a lossless interferometer such that $\bar{n}_{0}=\left\langle\hat{n}_{a}\right\rangle+\left\langle\hat{n}_{b}\right\rangle$ is conserved) leading to the average value $\left\langle 2 \hat{J}_{z}\right\rangle=\bar{n}_{0} \cos \phi$. It is also straight-forward to show that $\left\langle\left(2 \hat{J}_{z}\right)^{2}\right\rangle=\bar{n}_{0}\left(1+\left\langle 2 \hat{J}_{z}\right\rangle \cos \phi\right)$. Combining these expressions and Eq. 60 yields a phase uncertainty

$$
\Delta \phi_{2 \hat{J}_{z}}=\frac{\sqrt{\left\langle\hat{J}_{z}^{2}\right\rangle-\left\langle\hat{J}_{z}\right\rangle^{2}}}{\left|\partial_{\phi}\left\langle\hat{J}_{z}\right\rangle\right|}=\frac{1}{\sqrt{\bar{n}_{0}}|\sin \phi|}
$$

which yields the SQL of phase sensitivity for the value of the phase $\phi=\pi / 2$, which means that the detection of small phase shifts, such as what would be expected in gravitational wave detectors, would have a high degree of uncertainty. Of course, one could compensate for this by inserting a $\pi / 2$ phase-shifting element which would have the effect of replacing the $\sin \phi$ with a $\cos \phi$ in Eq. 104 . It is worth pointing out that the fact that $\Delta \hat{J}_{z}$ does not vanish is an indication that the quantum fluctuations of the vacuum (note the coherent state has the same quantum fluctuations as the vacuum) has the effect of limiting the precision of the phase-shift measurement. Next we consider the use of parity, performed on the output $b$-mode, as our detection observable.

\section{Parity-based detection}

We define parity with respect to the $b$-mode as $\hat{\Pi}_{b}=(-1)^{\hat{b}^{\dagger} \hat{b}}$ and likewise for the $a$-mode $\hat{\Pi}_{a}=(-1)^{\hat{a}^{\dagger} \hat{a}}$. From this the corresponding expectation values and their first derivatives are found to be

$$
\begin{gathered}
\left\langle\hat{\Pi}_{a(b)}\right\rangle=e^{-\bar{n}_{0}(1 \pm \cos \phi)}, \\
\partial_{\phi}\left\langle\hat{\Pi}_{a(b)}\right\rangle= \pm \bar{n}_{0} \sin \phi\left\langle\hat{\Pi}_{a(b)}\right\rangle,
\end{gathered}
$$

Noting that $\left\langle\hat{\Pi}_{a(b)}^{2}\right\rangle \equiv 1$, the phase uncertainty can immediately be found from the error propagation calculus

$$
\begin{gathered}
\Delta \phi_{\hat{\Pi}_{a}}=\frac{\sqrt{1-\left\langle\hat{\Pi}_{a}\right\rangle^{2}}}{\left|\partial_{\phi}\left\langle\hat{\Pi}_{a}\right\rangle\right|}=\frac{\sqrt{e^{4 \bar{n}_{0} \cos ^{2} \phi / 2}-1}}{\bar{n}_{0}|\sin \phi|} \\
\Delta \phi_{\hat{\Pi}_{b}}=\frac{\sqrt{1-\left\langle\hat{\Pi}_{b}\right\rangle^{2}}}{\left|\partial_{\phi}\left\langle\hat{\Pi}_{b}\right\rangle\right|}=\frac{\sqrt{e^{4 \bar{n}_{0} \sin ^{2} \phi / 2}-1}}{\bar{n}_{0}|\sin \phi|}
\end{gathered}
$$

The curves for both $\left\langle\hat{\Pi}_{a(b)}\right\rangle$ are displaced from one another by a $\pi$-phase shift, as evident by Eq. 105 . This implies that while the peak for $\left\langle\hat{\Pi}_{b}\right\rangle$ occurs at $\phi=0$, the peak for $\left\langle\hat{\Pi}_{a}\right\rangle$ occurs at $\phi=\pi$. We can expand Eqs. 107 and 108 about their respective optimal phase values to find

$$
\begin{gathered}
\Delta \phi_{\hat{\Pi}_{a}} \stackrel{\phi \rightarrow \pi}{\simeq} \frac{1}{\sqrt{\bar{n}_{0}|\cos \phi|}}, \\
\Delta \phi_{\hat{\Pi}_{b}} \stackrel{\phi \rightarrow 0}{\simeq} \frac{1}{\sqrt{\bar{n}_{0}|\cos \phi|}},
\end{gathered}
$$

which is in agreement with the minimum phase uncertainty attainable for this choice of initial state, the qCRB. This can be quickly verified by calculating the QFI using Eq. 54 to immediately give the SQL $\Delta \phi_{\min }=1 / \sqrt{\bar{n}_{0}}=\Delta \phi_{\mathrm{SQL}}$. 


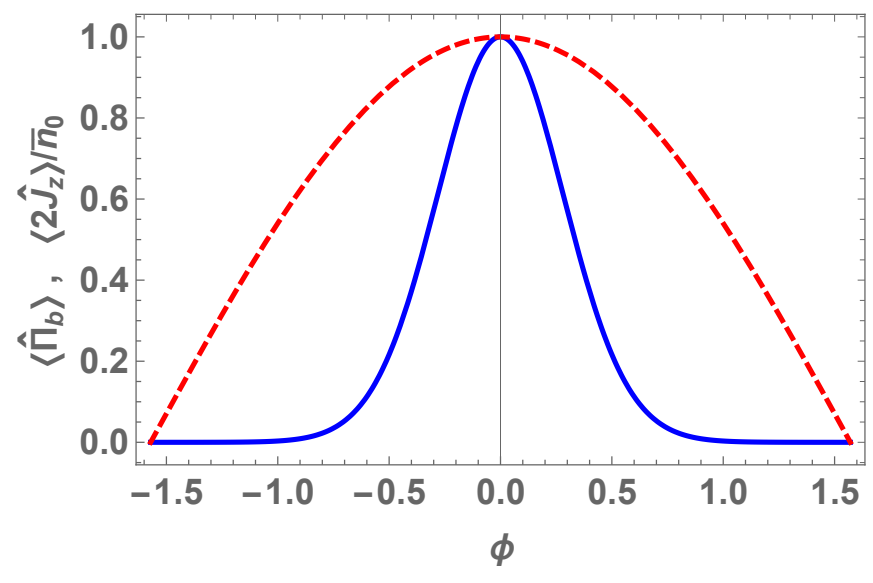

FIG. 11. Comparison of measurement resolution obtained for an input state $\mid$ in $\rangle=|\alpha\rangle_{a} \otimes|0\rangle_{b}$ between scaled intensity-difference $\left\langle 2 \hat{J}_{z}\right\rangle / \bar{n}_{0}$ (red, dashed) and parity $\left\langle\hat{\Pi}_{b}\right\rangle$ (blue, solid). The coherent state amplitude is taken to be $|\alpha|=5$.

The output signal itself is not super-resolved in the usual sense of having $N \phi(N>1)$ oscillation frequency scaling. However, the narrowing of the peak when considering parity-based measurement as opposed to the usual method of taking the intensity-difference has been defined as a form of super-resolution by Gao et a 67 . It is worth pointing out that for input states displaying quantum properties, such as all of the cases considered in the previous sections, super-resolution tends towards providing a greater degree of phase sensitivity. On the other hand, super-resolution has also been demonstrated in the absence of entangled states using light exhibiting strictly classical interference 69 . We also note the method of photon-number parity detection has recently been discussed in the context of SU $(1,1)$ interferometry 70 .

\section{EXPERIMENTAL REALIZATIONS OF PARITY-BASED DETECTION}

As emphasized in the Introduction, the parity operator, whether in the context of atomic (or spin) systems or in the context of photon number, is a Hermitian operator and is therefore an observable; but one that does not have a classical analog. The being the case, the question becomes how can parity be measured or least determined through some measurement process? The obvious way to do that is through counting the number of atoms ${ }^{71}$ in the excited (or ground), something that can be done through a process known as electron shelving [], or counting the number of photons in an optical field and raising -1 to that power. Of course, this means that the counting itself must be possible with a resolution at the level of a single atom or photon: a challenging prospect for the cases where the number or atoms or photons is large. Ideally one would like to be able to determine parity directly, by which we mean through a technique with a readout of \pm 1 without directly measuring the number of atoms or photons.
This could possibly be done with quantum non-demolition (QND) measurements. It is worth noting that QND measurements can be used to measure the number of atoms or photons as well, but what we have in mind is the measurement of parity wherein the detector does no counting at all. As we will show, these two mentioned methods of measuring parity, while ultimately yielding the same parity values, amounts to different kinds of measurements if used to perform, for example, stateprojective measurements.

\section{A. QND measures of atomic parity}

Here we will discuss several methods of performing a QND measure of atomic parity through the use of coupling between the atomic system with an ancillary subsystem. We begin by defining the even/odd atomic parity projection operators. That is, we define the operators that project the atomic state into even/odd numbers of excitations. These projectors are given by

$$
\begin{aligned}
\hat{\Pi}_{\mathrm{even}} & =\sum_{m=-j}^{j} \cos ^{2}\left[\frac{(j+m) \pi}{2}\right]|j, m\rangle\langle j, m|, \\
\hat{\Pi}_{\mathrm{odd}} & =\sum_{m=-j}^{j} \sin ^{2}\left[\frac{(j+m) \pi}{2}\right]|j, m\rangle\langle j, m|,
\end{aligned}
$$

satisfying the POVM condition $\sum_{i} \hat{\Pi}_{i}=\hat{I}$. Note that $j+m$ represents the total number of atoms found in the excited state while $j-m$ represents the total number of atoms found in the ground state. Consequently $j \pm m$ takes on only integer values such that for a given $j \pm m$, only one of the projectors will be nonzero. These projection operators can be used to express the atomic parity operator as

$$
\begin{aligned}
\hat{\Pi} & =\sum_{i} \lambda_{i} \hat{\Pi}_{i}=\hat{\Pi}_{\mathrm{even}}-\hat{\Pi}_{\mathrm{odd}} \\
& =\sum_{m=-j}^{j} \cos [(j+m) \pi]|j, m\rangle\langle j, m|
\end{aligned}
$$

where $\lambda_{i}$ are the eigenvalues of the parity operator, respectively. From Eqs. 111 and 112 , it follows that

$$
\begin{aligned}
& \left\langle\hat{\Pi}_{\text {even }}\right\rangle=\frac{1}{2}(1+\langle\hat{\Pi}\rangle), \\
& \left\langle\hat{\Pi}_{\text {odd }}\right\rangle=\frac{1}{2}(1-\langle\hat{\Pi}\rangle),
\end{aligned}
$$

where $\left\langle\hat{\Pi}_{\text {odd }}\right\rangle+\left\langle\hat{\Pi}_{\text {even }}\right\rangle=1$. We note here that 'parity' is defined with respect to the number of atoms found in the excited state. That is, 'even' parity denotes an even number of atoms 
found in the excited state and 'odd' parity denotes and odd number of atoms found in the excited state. Let us move on to consider a couple of different cases.

\section{Coupling to an ancillary atomic system}

Given an atomic system in which we wish to measure, denoted by the $a$-mode, we introduce an ancillary atomic system (occupying the $b$-mode) prepared in an atomic coherent state $\left|\tau, j_{b}\right\rangle_{b}$ as well as the coupling Hamiltonian and corresponding evolution operator:

$$
\hat{H}=\hbar \chi\left(\hat{J}_{0}^{a}+\hat{J}_{z}^{a}\right)\left(\hat{J}_{0}^{b}+\hat{J}_{z}^{b}\right) \quad \rightarrow \quad \hat{U}=e^{-i t \hat{H} / \hbar},
$$

where $\chi$ is the coupling strength. This interaction Hamiltonian can be thought of as the atomic analog to the field coupling cross-Kerr interaction. We define the initial state as

$$
\begin{aligned}
\mid \text { initial }\rangle & =|\psi\rangle_{a} \otimes\left|\tau, j_{b}\right\rangle_{b}=\sum_{m=-j_{a}}^{j_{a}} C_{m}^{\left(j_{a}\right)}\left|j_{a}, m\right\rangle_{a} \otimes \\
& \otimes\left(1+|\tau|^{2}\right)^{-j_{b}} \sum_{m^{\prime}=-j_{b}}^{j_{b}}\left(\begin{array}{c}
2 j_{b} \\
j_{b}+m^{\prime}
\end{array}\right) \tau^{j_{b}+m^{\prime}}\left|j_{b}, m^{\prime}\right\rangle_{b} .
\end{aligned}
$$

Note that we wish to make a projective measurement on the ancillary atomic system ( $b$-system) in order to determine the atomic parity of the target system ( $a$-system). The final state is then

$$
\begin{aligned}
\mid \text { final }\rangle & \left.=e^{-i t \hat{H} / \hbar} \mid \text { initial }\right\rangle \\
& =\sum_{m=-j_{a}}^{j_{a}} C_{m}^{\left(j_{a}\right)}\left|j_{a}, m\right\rangle_{a} \otimes\left|\tau e^{-i t \chi\left(j_{a}+m\right)}, j_{b}\right\rangle_{b} .
\end{aligned}
$$

For the choice of $\chi t \rightarrow \pi$, this becomes

$$
\begin{aligned}
\mid \text { final }\rangle=\left|\psi_{\text {even }}\right\rangle_{a}\left|\tau, j_{b}\right\rangle_{b}+\left|\psi_{\text {odd }}\right\rangle_{a}\left|-\tau, j_{b}\right\rangle_{b} \\
=\sum_{m=-j_{a}}^{j_{a}}\left[\cos ^{2}\left(\frac{\left(j_{a}+m\right) \pi}{2}\right)\left|\tau, j_{b}\right\rangle_{b}+\right. \\
\left.\quad+\sin ^{2}\left(\frac{\left(j_{a}+m\right) \pi}{2}\right)\left|-\tau, j_{b}\right\rangle_{b}\right] C_{m}^{\left(j_{a}\right)}\left|j_{a}, m\right\rangle \\
=\sum_{m=-j_{a}}^{j_{a}} C_{m}^{\left(j_{a}\right)}\left|j_{a}, m\right\rangle_{a}\left|\Phi_{m}\right\rangle_{b} .
\end{aligned}
$$

The system is entangled such that a projection onto the ancillary atomic system yielding the ACS characterized by parameter $-\tau$ will project out odd atomic states in the target system and projection onto the ACS characterized by parameter $+\tau$ will project out even atomic states in the target system. What we require is a means of determining which state the ancillary atomic system is in. Let us assume the ancillary atomic coherent state is prepared such that $\tau=-1$; this corresponds to a separable state in which all atoms of the ancillary atomic system are in the same superposition state

$$
e^{-i \frac{\pi}{2} \hat{J}_{y}}|j,-j\rangle=|-1, j\rangle=\left|\psi_{-}\right\rangle^{\otimes N}=\frac{1}{2^{N / 2}}(|g\rangle-|e\rangle)^{\otimes N} .
$$

Similarly, we can also define the phase-rotated atomic coherent state

$$
e^{-i \frac{\pi}{2} \hat{J}_{y}}|j, j\rangle=|1, j\rangle=\left|\psi_{+}\right\rangle^{\otimes N}=\frac{1}{2^{N / 2}}(|g\rangle+|e\rangle)^{\otimes N} .
$$

With this, we can rewrite the state $\left|\Phi_{m}\right\rangle_{b}$, assuming $\tau=-1$, as

$$
\begin{aligned}
\left|\Phi_{m}\right\rangle_{b}= & \cos ^{2}\left(\frac{\left(j_{a}+m\right) \pi}{2}\right)\left|-1, j_{b}\right\rangle_{b}+\sin ^{2}\left(\frac{\left(j_{a}+m\right) \pi}{2}\right)\left|1, j_{b}\right\rangle_{b} \\
= & \cos ^{2}\left(\frac{\left(j_{a}+m\right) \pi}{2}\right) e^{-i \frac{\pi}{2} \hat{J}_{y}^{b}}\left|j_{b},-j_{b}\right\rangle_{b}+ \\
\quad & \sin ^{2}\left(\frac{\left(j_{a}+m\right) \pi}{2}\right) e^{-i \frac{\pi}{2} \hat{J}_{y}^{b}}\left|j_{b}, j_{b}\right\rangle_{b} .
\end{aligned}
$$

It is important to note that only a single term in the superposition state Eq.122 can be present at a time. Performing a single $\pi / 2$-pulse yields the ancillary atomic system in the state

$$
e^{i \frac{\pi}{2} \hat{J}_{y}^{b}}\left|\Phi_{m}\right\rangle_{b}= \begin{cases}\cos ^{2}\left(\frac{\left(j_{a}+m\right) \pi}{2}\right)\left|j_{b},-j_{b}\right\rangle_{b}, & j_{a}+m \text { even } \\ \sin ^{2}\left(\frac{\left(j_{a}+m\right) \pi}{2}\right)\left|j_{b}, j_{b}\right\rangle_{b}, & j_{a}+m \text { odd }\end{cases}
$$

where $j_{a}+m$ is an integer corresponding to the number of excited atoms. A state-reductive measurement performed on the ancillary atomic system would inform the experimenter of the parity of the target system without providing explicit knowledge of the value of $j_{a}+m$. Note that this works for an arbitrary value of $j_{b}$; the ancillary atomic system can be as small as a single atom. Next we will discuss a method involving a known coupling Hamiltonian readily capable of being experimentally implemented.

\section{Coupling to a field state}

Some work has been done in developing a QND measure of photon number parity and a means of projecting out parity eigenstates in optical fields72. Similarly, QND parity measurements have been performed in the context of error correction for a hardware-efficient protected quantum memory using Schrödinger cat states ${ }^{73}$ as well as a fault-tolerant detection of quantum error ${ }^{74}$. Parity measurements have also been utilized in the entanglement of bosonic modes through the realization of the eSWAP operation ${ }^{75}$ using bosonic qubits stored in two superconducting microwave cavities ${ }^{76}$. Here will consider a 
similar scheme in which one couples the target atomic system to an ancillary field state. The interaction Hamiltonian coupling the two subsystems is given by

$$
\hat{H}=\hbar \chi \hat{b}^{\dagger} \hat{b} \hat{J}_{z} \quad \rightarrow \quad \hat{U}=e^{-i \frac{t}{\hbar} \hat{H}}
$$

where $\left\{\hat{b}, \hat{b}^{\dagger}\right\}$ are the usual boson annihilation and creation operators, respectively. Consider an ancillary field state, given by the usual coherent state

$$
|\alpha\rangle_{b}=e^{-\frac{1}{2}|\alpha|^{2}} \sum_{n=0}^{\infty} \frac{\alpha^{n}}{\sqrt{n !}}|n\rangle_{b}
$$

Following the same procedure as the previous section and setting $\chi t=\pi$ this coupling Hamiltonian yields the final state

$$
\mid \text { final }\rangle=\hat{U} \mid \text { initial }\rangle=\sum_{m=-j}^{j} C_{m}^{(j)}|j, m\rangle_{a} \otimes\left|(-1)^{m} \alpha\right\rangle_{b}
$$

The final entangled state will depend greatly on the value of $j$. More specifically, noting $N=2 j$, this simplifies to

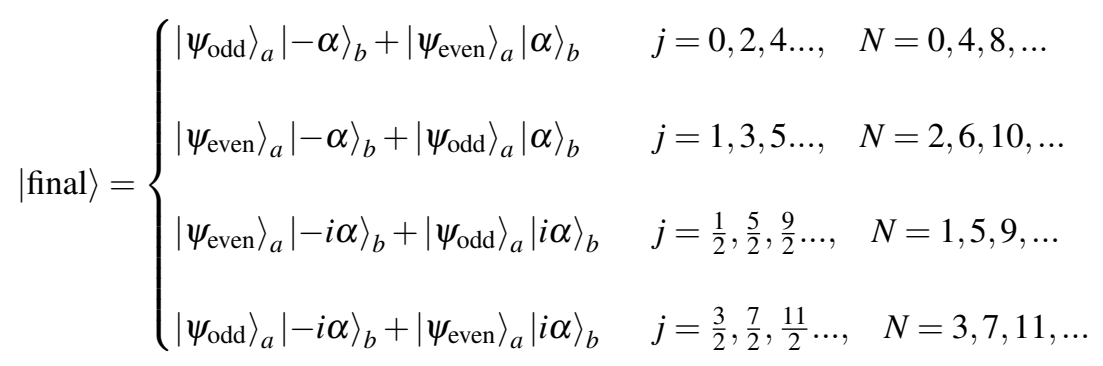

In order for this procedure to be applicable, the total number of atoms involved must be known with certainty as the resulting superposition state and subsequent detection scheme will greatly depend on having this information. As a proof of concept, let us consider the case where the number of atoms is $N=0,4,8, \ldots$. As per Eq. 127, the final state is given by $\mid$ final $\rangle=\left|\psi_{\text {odd }}\right\rangle_{a}|-\alpha\rangle_{b}+\left|\psi_{\text {even }}\right\rangle_{a}|\alpha\rangle_{b}$. Mixing the field at a 50:50 beam splitter with an equal-amplitude phase-adjusted coherent state $|\alpha\rangle_{c}$. Assuming a $50: 50 \hat{J}_{y}$-type beam splitter of angle $-\pi / 2$ (see Appendix A 3, the beam splitter (labeled BS 2 for reasons that will become clear) results in the transformation

$$
\begin{gathered}
|\alpha\rangle_{b}|\alpha\rangle_{c} \stackrel{\text { BS } 2}{\longrightarrow}|\sqrt{2} \alpha\rangle_{b}|0\rangle_{c}, \\
|-\alpha\rangle_{b}|\alpha\rangle_{c} \stackrel{\text { BS } 2}{\longrightarrow}|0\rangle_{b}|\sqrt{2} \alpha\rangle_{c} .
\end{gathered}
$$

The total state is now given by

$$
\mid \text { final }\rangle_{\mathrm{BS} 2}=\left|\psi_{\mathrm{odd}}\right\rangle_{a}|0\rangle_{b}|\sqrt{2} \alpha\rangle_{c}+\left|\psi_{\text {even }}\right\rangle_{a}|\sqrt{2} \alpha\rangle_{b}|0\rangle_{c}
$$

in which all photons are in either one optical mode or the other. A simple detection scheme informs the experimenter of the parity of the target atomic system. Upon a state reductive measurement performed on the optical modes, the atomic system becomes

$$
|\Psi\rangle_{a}= \begin{cases}\left|\psi_{\text {even }}\right\rangle_{a}, & b \text {-mode click } \\ \left|\psi_{\text {odd }}\right\rangle_{a}, & c \text {-mode click }\end{cases}
$$

The optical fields $|\alpha\rangle_{b}$ and $|\alpha\rangle_{c}$ used in this procedure can be derived from the same beam if one starts with the state $|\sqrt{2} \alpha\rangle_{c}$ and uses a $50: 50 \hat{J}_{y}$-type beam splitter of angle $+\pi / 2$ such that

$$
|\sqrt{2} \alpha\rangle_{b}|0\rangle_{c} \stackrel{\mathrm{BS} 1}{\longrightarrow}|\alpha\rangle_{b}|\alpha\rangle_{c}
$$

After beamsplitting, the $b$-mode coherent state can be coupled with the target atomic system before being photomixed with the coherent state $|\alpha\rangle_{c}$ at the second beam splitter. This method would allow one to determine the parity of the atomic system without explicit knowledge of the number of exicted atoms $j+m$.

\section{B. Detection of photon parity}

As mentioned above, one way to obtain photon number parity is perform photon number counts $n_{\text {count }}$ and raise -1 to that power: $\Pi_{\text {measured }}=(-1)^{n_{\text {count }}}$. That raises the issue of the general lack of photon number counting techniques having resolution at the level of one photon. Yet there are ways around this problem. In fact, an experiment to detect a phase shift through optical interferometry using coherent 


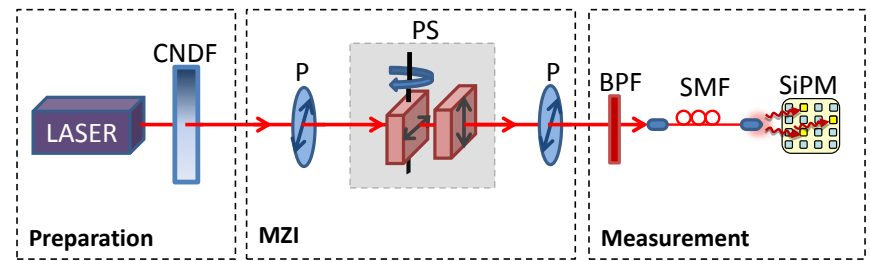

FIG. 12. A schematic of the experimental setup used by Cohen et al. The coherent states are produced by a Ti:Sapphire laser with average photon numbers controlled by a calibrated neutral density filer (NDF) and the MZI is composed from two polarizers $P$ at $45^{\circ}$ and a phase shifter. What is defined in the text as the output $a$-mode is filtered spectrally by $3 \mathrm{~nm}$ band pass filter (BPF) and spatially by a single mode fiber (SMF). This mode is detected by the silicon photomultiplier. Figure taken from Cohen et al ${ }^{77}$ with permission from the authors.

light and parity measurements was performed a few years ago by Eisenberg's group at Hebrew University 77 . Recall from above, and from Gao et al. ${ }^{67}$, that parity measurements performed in this context are predicted to result in phase-shift detections that are super-resolved even though the phase sensitivity is at the SQL. The experiment of L. Cohen et al ${ }^{77}$ confirm this.

In the experiment reported, the phase shift to be detected was set to be $\pi$. If we look at Eqs. 107 and 108, and based on the discussion to follow, we see that with our labeling scheme, we should be performing parity measurements on the output $a$-mode. That is, for $\phi$ near $\pi$, we have $\left\langle\hat{\Pi}_{a}\right\rangle=e^{-\bar{n}_{0}(1+\cos \phi)} \simeq e^{-\frac{\bar{n}_{0}}{2}(\phi-\pi)^{2}}$, where $\bar{n}_{0}$ is the total number of photons within the interferometer, which peaks at unity for $\phi \rightarrow \pi$. In this limit $\Delta \phi \rightarrow 1 / \sqrt{\bar{n}_{0}}$, the SQL.

These authors also measured a different kind of parity in which the outcomes are either no photons detected, as described by the projector $|0\rangle\langle 0|$, or any number of photons detected but without resolution, $\sum_{n=0}^{\infty}|n\rangle\langle n|=\boldsymbol{I}-| 0\rangle\langle 0|$. Defining $\hat{Z}=|0\rangle_{a}\langle 0|$, from the output state given in Eq. 103 we find the probability of there being no photon detections to be

$$
P_{0}(\phi)=\langle\hat{Z}\rangle_{\text {out }}=e^{-\frac{\bar{n}_{0}}{2}(1+\cos \phi)} \stackrel{\phi \rightarrow \pi}{\simeq} e^{-\frac{\bar{n}_{0}}{4}(\phi-\pi)^{2}} .
$$

From the error propagation calculus one easily finds for $\phi \rightarrow \pi$, the phase uncertainty is given by $\Delta \phi_{a} \rightarrow 1 / \sqrt{\bar{n}_{0}}$, which is also shot-noise limited. Evidently the two observables give similar results for the case of input coherent light.

The experimental setup used by Cohen et al ${ }^{771}$ is detailed in Fig. 12 The coherent inputs are produced by a Ti:Sapphire laser with a calibrated variable neutral density filter (NDF) employed to control the average photon numbers. The MZI consists of two polarizers at $45^{\circ}$ and the phase-shift is produced by tilting the calcite crystal. The output mode to be measured is band pass filtered (BPF) and spatially filtered by a single mode fiber (SMF). This mode was detected by a silicon photomultiplier consisting of an array of beam

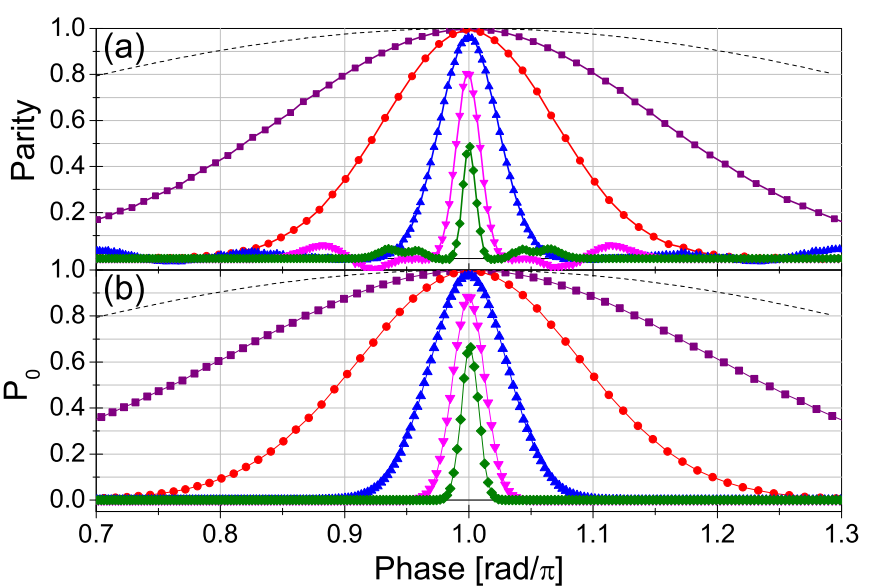

FIG. 13. Expectation values of the parity operator as well as the no-photons-detected projector $\hat{Z}$. For each curve the average photon numbers are: $4.6 \pm 0.2$ (purple, square), $25 \pm 1$ (red, circle), $200 \pm 8$ (blue, triangle), 1, 190 \pm 50 (pink, inverted triangle) and 4, 150 \pm 150 (green, rhombus). The classic interference curves are presented in black-dashed lines on each plot. Errors are not shown as they are too small to be represented. Figure taken from Cohen et al ${ }^{[77}$ with permission from the authors.

splitters and single-photon detectors. Such an arrangement for photon counting with a resolution at the single-photon level is described in Kok and Lovett ${ }^{78}$. See also the review of photon detection by Silberhorn 79 . For the fine details of the experiment, we refer the reader to the paper by Cohen et al. ${ }^{177}$.

The expectation values of the parity operator $\hat{\Pi}_{a}$ (in our notation) and $\hat{Z}$ are plotted in Fig. 13 for several values of average photon number. The predicted narrowing around the peak at $\phi=\pi$ is evident, though the degradation of the visibility for high average photon number is due to the imperfect visibility of the interferometer itself as well as dark counts. Plots of the corresponding phase uncertainties for $\bar{n}_{0}=200$ are found in Fig. 14 . Note that the peak at $\phi=\pi$ is wider for $P_{0}$ than for parity. Finally in Fig. 15 the results are summarized for resolution and sensitivty showing that, in part (a), parity yields greater resolution thatn $P_{0}$. That is, for parity-based measurement, resolution reachs $\frac{\lambda}{288}$ where $\lambda=780 \mathrm{~nm}$ is the wavelength of the laser light, which is a factor of $1 / 144$ improvement (smaller) over the Rayleigh limit $\lambda / 2$. The resolutions in the two cases differ by the expected amount $\sqrt{2}$, On the other hand, as can be seen in part (b) of Fig. 15, parity has a larger deviation from the SQL as compared to $P_{0}$. In fact, up to 200 photons, sensitivity of $P_{0}$ is maintained at the SQL.

We mention that in recent years considerable effort has been directed towards the development of photon-numberresolving detectors. These include superconducting transition edge detectors 80 , loop detectors 81 , detection by multiplexing 82 , an array of avalanche photodiodes (APDs) and an array of single-photon detectors 84 . This list by no 


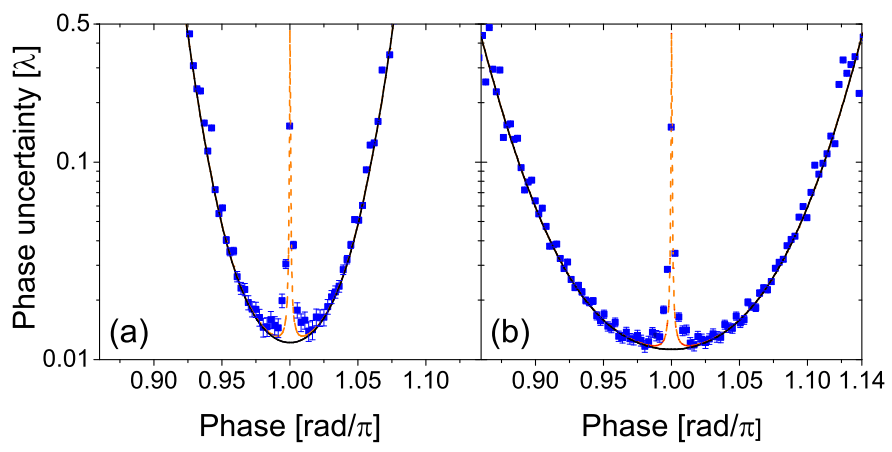

FIG. 14. Phase uncertainties obtained through experimental data for $\bar{n}_{0}=200$ (blue, square) as a function of the phase $\phi$ for both parity (left-most) and no-photons-detected probability $P_{0}$ (right-most). Fits based on the theory are represented by solid black lines and a correction made to account for imperfect visibility (see Eq. 9 in Cohen $e t$ $a l{ }^{[77}$ ) by orange-dashed lines. Errors representing the standard deviation in the phase uncertainty estimation process are shown when larger than their symbols. Figure taken from Cohen et al ${ }^{[7]}$ with permission from the authors.

means exhausts the literature on this topic. In principle one could avoid photon counting entirely and instead perform a quantum non-demolition measurement of photonic parity in the same vain as discussed in Section $\mathrm{VA}$ pertaining to atomic parity. This possibility has been discussed by Gerry et $a l^{[72]}$ as an extension of a technique proposed for the QND measurement of photon number ${ }^{85}$. The problem with these techniques is that they depend on a cross-Kerr interaction with a large third order nonlinearity which does not exist in optical materials.

But there is yet another possibility first mentioned by Campos et al ${ }^{[49}$ in their analysis of interferometry with twin-Fock states. Recall that the Wigner function is given by $W(\alpha)=$ $\frac{2}{\pi}\left\langle\hat{D}(\alpha) \hat{\Pi} \hat{D}^{\dagger}(\alpha)\right\rangle$; it follows for $\alpha=0$ that $\langle\hat{\Pi}\rangle \equiv \frac{\pi}{2} W(0)$, thus showing the expectation value of the parity operator can be found directly by value of the Wigner function at the origin of phase space. Now the Wigner function can be constructed by the techniques of quantum state tomography which uses a balanced homodyning and the inverse Radon transformation to perform filtered back projections ${ }^{4}$. However, one does not need the entire Wigner function: only its value at the origin of phase space is required. Plick et al ${ }^{86}$ have examined this prospect in detail for Gaussian states of light.

\section{Quantum random number generator}

We close this section with a brief discussion of the prospect of using photon-number resolved detection as a basis for a quantum random number generator (QRNG) (for a discussion on QRNGs, see Herrero-Collantes et al ${ }^{87}$ and references therein) with laser light. As is well known, laser light shone on a 50:50 beam splitter cannot be the basis of a QRNG as a matter of principle no matter the intensity of the light. The ideal source of light is a single photon generated on demand,

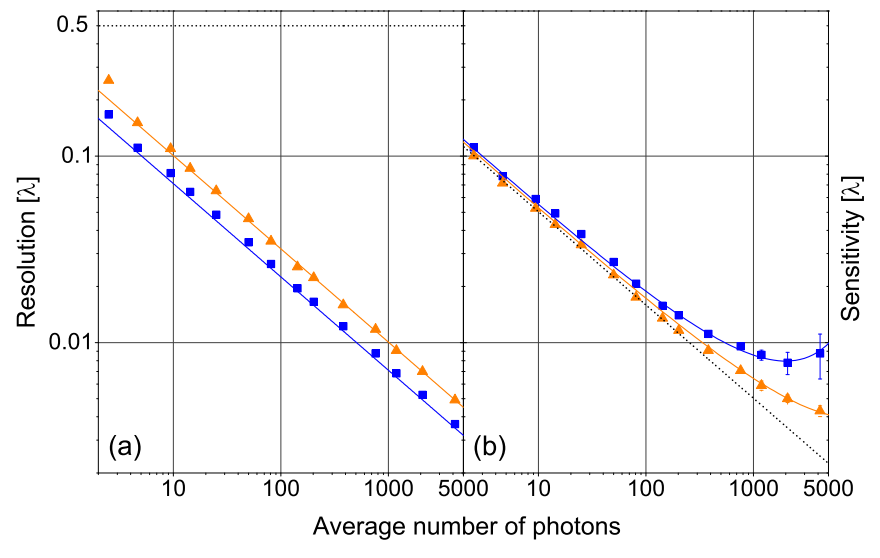

FIG. 15. (a) The resolution and (b) the smallest uncertainty, plotted as a function of average photon number. Parity results are represented by blue squares and $P_{0}$ with orange triangles. Solid lines are the theoretical predictions. For the resolution there are no free parameters and the predictions for sensitivity use the visibility values from the fits of Fig. 14 (the orange-dashed lines). Parity has better resolution but larger deviation from the SQL. Errors were calculated as before and shown when larger than their symbol. Figure taken from Cohen et al ${ }^{[7]}$ with permission from the authors.

where the photon falls on the beam splitter and then appears randomly, i.e. is detected randomly, in one or the other output beams. Since on-demand single photon generation is problematic in practice, QRNG devices use weak (coherent) laser light; the idea being that on average only one photon is present at any given time. This is a fiction, of course, as the probability of having two photons at a time is not zero due to the photon bunching effect ${ }^{68}$. Now consider light prepared in a coherent state $|\alpha\rangle$ by a well phase-stabilized laser. The expectation value of the parity operator is

$$
\langle\alpha|\hat{\Pi}| \alpha\rangle=e^{-|\alpha|^{2}} \sum_{n=0}^{\infty} \frac{|\alpha|^{2 n}}{n !}(-1)^{n}=e^{-\bar{n}} \sum_{n=0}^{\infty} \frac{(-\bar{n})^{n}}{n !}=e^{-2 \bar{n}}
$$

where $\bar{n}=|\alpha|^{2}$. It is easy to show numerically that $\langle\hat{\Pi}\rangle \rightarrow 0$ as $\bar{n}$ becomes large. Another way to put it is that the probabilities of getting even or odd photon-number parity must be equal to $1 / 2$ for sufficiently large $\bar{n}$. For arbitrary $\alpha$ these probabilities are

$$
\begin{aligned}
P_{e}=\left\langle\alpha\left|\hat{P}_{e}\right| \alpha\right\rangle & =e^{-|\alpha|^{2}} \sum_{m=0}^{\infty} \frac{|\alpha|^{4 m}}{(2 m) !} \\
& =e^{-\bar{n}} \sum_{m=0}^{\infty} \frac{\bar{n}^{2 m}}{(2 m) !}=e^{-\bar{n}} \cosh \bar{n} \\
P_{o}=\left\langle\alpha\left|\hat{P}_{o}\right| \alpha\right\rangle & =e^{-|\alpha|^{2}} \sum_{m=0}^{\infty} \frac{|\alpha|^{4 m+2}}{(2 m+1) !} \\
& =e^{-\bar{n}} \sum_{m=0}^{\infty} \frac{\bar{n}^{2 m+1}}{(2 m+1) !}=e^{-\bar{n}} \sinh \bar{n}
\end{aligned}
$$


where the even and odd photon number projectors are given, respectively, by $\hat{P}_{e}=\sum_{m=0}^{\infty}|2 m\rangle\langle 2 m|$ and $\hat{P}_{o}=$ $\sum_{m=0}^{\infty}|2 m+1\rangle\langle 2 m+1|$ and where

$$
\langle\hat{\Pi}\rangle=\left\langle\hat{P}_{e}\right\rangle-\left\langle\hat{P}_{o}\right\rangle=P_{e}-P_{o}=e^{-2 \bar{n}} .
$$

The average photon number need not be very high for the probabilities in Eq. 134 and 135 to equalize close to $1 / 2$. In fact, for $\bar{n}=9$ one has $P_{e}=P_{o}=0.5$ after retaining seven decimal places. For $\bar{n}=16$, this is true to thirteen decimal places. Thus a viable QRNG could be implemented with efficient parity detection in a regime where the average photon number need not be very high.

Lastly we mention that laser light prepared in a pure coherent state is not necessary: a statistical mixture of coherent states of the same amplitude will suffice. Consider the phase-averaged coherent states given by the density operator

$$
\rho=\frac{1}{2 \pi} \int_{0}^{2 \pi} d \phi\left|r e^{i \phi}\right\rangle\left\langle r e^{i \phi}\right|,
$$

where $r=|\alpha|=\sqrt{\bar{n}}$. It is easily shown that

$$
\begin{aligned}
\langle\hat{\Pi}\rangle=\operatorname{Tr}[\rho \hat{\Pi}] & =\frac{1}{2 \pi} \int_{0}^{2 \pi} d \phi \sum_{n=0}^{\infty}(-1)^{n}\left|\left\langle n \mid r e^{i \phi}\right\rangle\right|^{2} \\
& =e^{-\bar{n}} \sum_{n=0}^{\infty} \frac{(-\bar{n})^{n}}{n !}=e^{-2 \bar{n}}
\end{aligned}
$$

which is identical to Eq. 133

In the next section we will discuss parity-based measurement in the context of multi-atom spectroscopy with a particular interest in the construction of high-precision atomic clocks. We will consider both the classical case in which the atoms are initially unentangled and prepared in the same initial state as well as the case in which the atoms are initially entangled and spin-squeezed. A comparison between parity and population difference is also made in the context of enhanced measurement resolution.

\section{ENHANCED MEASUREMENT RESOLUTION IN MULTI-ATOM ATOMIC CLOCKS}

We have discussed the benefit of parity-based detection in the context of measurement resolution in two-mode optical systems as it pertains to interferometric measurements. Enchanced measurement resolution may also be beneficial in the construction of highly precise multi-atom atomic clocks. Collections of two level atoms have seen use in quantum information $\frac{88}{1}$ (in the context of cold atoms) and quantum metrology 89 . In this section we will endeavor to show how parity-based measurements yields finer resolution for increasingly large numbers of atoms whereas simply considering the population difference between ground and excited states does not. We will initially restrict the discussion to unentangled ensembles of atoms, where each atom is initially prepared in the same atomic state, before considering the case of an initially entangled ensemble. The latter case provides even greater resolution when parity measurements are performed.

\section{A. Population difference for an arbitrary atomic state}

Consider a general atomic state expressed in terms of a superposition of all Dicke states $|j, m\rangle$, where the Dicke states are defined relative to the individual atomic states in the previous section, given by

$$
\left|\psi_{\pi / 2}\right\rangle=\sum_{m=-j}^{j} C_{m}^{(j)}|j, m\rangle .
$$

Let Eq. 139 describe the state after the first $\pi / 2$-pulse in a Ramsey interferometer, where $C_{m}^{(j)}$ are the probability amplitudes for obtaining each state $|j, m\rangle$. The state after the time evolution and the final $\pi / 2$-pulse is given by

$$
\begin{aligned}
\left|\Psi_{\mathrm{F}}\right\rangle & =e^{-i \frac{\pi}{2} \hat{J}_{y}} e^{-i \phi \hat{J}_{z}}\left|\psi_{\pi / 2}\right\rangle \\
& =\sum_{m^{\prime}=-j}^{j}\left[\sum_{m=-j}^{j} C_{m}^{(j)} e^{-i \phi m} d_{m^{\prime}, m}^{j}\left(\frac{\pi}{2}\right)\right]\left|j, m^{\prime}\right\rangle \\
& =\sum_{m^{\prime}=-j}^{j} \tilde{C}_{m^{\prime}}^{(j)}\left|j, m^{\prime}\right\rangle
\end{aligned}
$$

where $\tilde{C}_{m^{\prime}}^{(j)}$ are defined within the square brackets of Eq. 140 and $d_{m^{\prime}, m}^{j}(\beta)$ are the usual Wigner- $d$ matrix elements given by $\left\langle j, m^{\prime}\left|e^{-i \beta \hat{J}_{y}}\right| j, m\right\rangle$. We consider the measurement resolution obtained for this general case when using $\left\langle\hat{J}_{z}\right\rangle$ as our detection observable. Physically, this corresponds to taking the difference between the number of atoms in the excited and ground states, respectively. The optical analog would be taking the difference in intensity between the two output modes of an MZI. The expectation value of $\hat{J}_{z}$ is given by

$$
\begin{aligned}
\left\langle\hat{J}_{z}\right\rangle & =\sum_{m=-j}^{j} \sum_{p=-j}^{j} C_{p}^{(j) *} C_{m}^{(j)} e^{i \phi(m-p)} \times \\
\times\left[\sum_{m^{\prime}=-j}^{j} m^{\prime} d_{m^{\prime}, p}^{j}\left(\frac{\pi}{2}\right) d_{m^{\prime}, m}^{j}\left(\frac{\pi}{2}\right)\right] & \\
= & \sum_{m=-j}^{j} \sum_{p=-j}^{j} C_{p}^{(j) *} C_{m}^{(j)} e^{i \phi(m-p)} \times \Gamma_{m, p}\left(\frac{\pi}{2}\right)
\end{aligned}
$$

where the function $\Gamma_{m, p}(\lambda)$ is defined by Eq. B14. Without loss of generality 90 , we assume the coefficients $C_{m}^{(j)}$ are real and we obtain the result

$$
\left\langle\hat{J}_{z}\right\rangle=\gamma^{(j)} \cos \phi+\alpha^{(j)}
$$




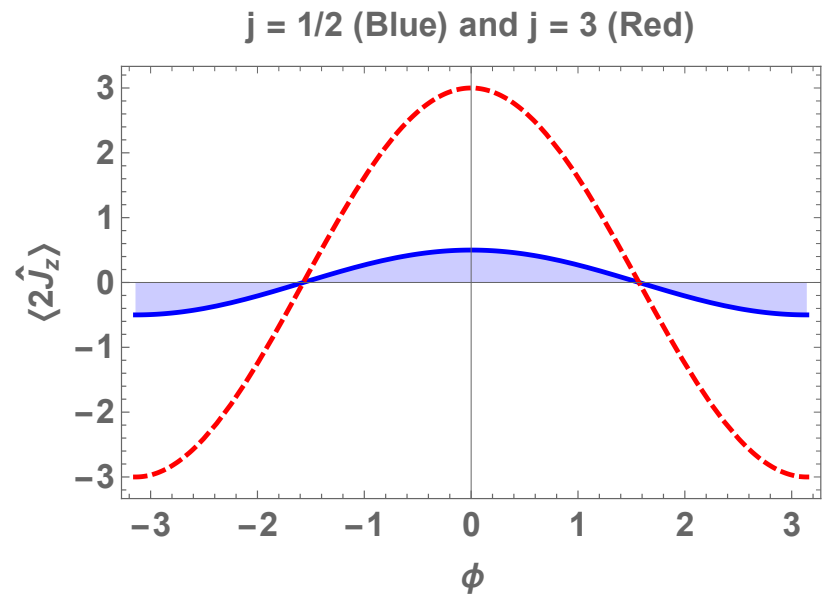

FIG. 16. $\left\langle\hat{J}_{z}\right\rangle$ versus $\phi$ for a $j=1 / 2$ (blue, solid) and $j=3$ (red, dashed). Note that we do not see greater resolution for larger numbers of atoms.

where

$$
\begin{aligned}
& \gamma^{(j)}=2 \sum_{m=-j}^{j} \sum_{m^{\prime}=-j}^{j} C_{m+1}^{(j)} C_{m}^{(j)} m^{\prime} d_{m^{\prime}, m+1}^{j}\left(\frac{\pi}{2}\right) d_{m^{\prime}, m}^{j}\left(\frac{\pi}{2}\right) \\
& \alpha^{(j)}=\sum_{m=-j}^{j} \sum_{m^{\prime}=-j}^{j}\left|C_{m}^{(j)} d_{m^{\prime}, m}^{j}\left(\frac{\pi}{2}\right)\right|^{2} m^{\prime}
\end{aligned}
$$

For the case of an initial atomic coherent state, this yields the expected result of Eq. 10

$$
\left\langle\hat{J}_{z}\right\rangle_{\mathrm{ACS}}=j \cos \phi,
$$

which we plot in Fig. 16 for several values of $j$. Similarly, an expression can be found for $\left\langle\hat{J}_{z}^{2}\right\rangle$ by using Eq. B15 which yields

$$
\left\langle\hat{J}_{z}^{2}\right\rangle_{\mathrm{ACS}}=\Omega^{(j)} \cos 2 \phi+\kappa^{(j)} \cos \phi+\beta^{(j)},
$$

where

$$
\begin{aligned}
\Omega^{(j)} & =2 \sum_{m=-j}^{j} \sum_{m^{\prime}=-j}^{j} C_{m+2}^{(j)} C_{m}^{(j)} m^{\prime 2} d_{m^{\prime}, m+2}^{j}\left(\frac{\pi}{2}\right) d_{m^{\prime}, m}^{(j)}\left(\frac{\pi}{2}\right), \\
\kappa^{(j)} & =2 \sum_{m=-j}^{j} \sum_{m^{\prime}=-j}^{j} C_{m+1}^{(j)} C_{m}^{(j)} m^{\prime 2} d_{m^{\prime}, m+1}^{j}\left(\frac{\pi}{2}\right) d_{m^{\prime}, m}^{j}\left(\frac{\pi}{2}\right), \\
\beta^{(j)} & =\sum_{m=-j}^{j} \sum_{m^{\prime}=-j}^{j}\left|C_{m}^{(j)} d_{m^{\prime}, m}^{j}\left(\frac{\pi}{2}\right) m^{\prime}\right|^{2},
\end{aligned}
$$

Note that in neither case do we see a narrowing of the peak regardless of the state used. The phase dependency goes as a cosine function (to the first power) regardless of the state coefficients and number of atoms used. This particular detection observable cannot yield enhanced measurement resolution regardless of the initial state under consideration.

\section{B. Parity-based resolution for an arbitrary atomic state}

Let us assume our state is initially prepared such that the state after the first $\pi / 2$ pulse is once again given by Eq. 139 . The action of the Ramsey interferometer is to transform this state to

$$
\begin{aligned}
\left|\Psi_{\mathrm{F}}\right\rangle & =e^{-i \frac{\pi}{2} \hat{J}_{y}} e^{-i \phi \hat{J}_{z}}\left|\psi_{\pi / 2}\right\rangle \\
& =\sum_{m=-j}^{j} \sum_{m^{\prime}=-j}^{j} C_{m}^{(j)} d_{m^{\prime}, m}^{j}\left(\frac{\pi}{2}\right) e^{-i \phi m}\left|j, m^{\prime}\right\rangle,
\end{aligned}
$$

where the Wigner- $d$ matrix elements $d_{m^{\prime}, m}^{j}(\beta)$ are discussed in Appendix $B$. The expectation value of the parity operator can be expressed as

$$
\begin{aligned}
\langle\hat{\Pi}\rangle= & \sum_{m=-j}^{j} \sum_{p=-j}^{j} C_{m}^{(j)} C_{p}^{(j) *} e^{-i \phi(m-p)} \times \\
& \times\left[\sum_{m^{\prime}=-j}^{j}(-1)^{j-m^{\prime}} d_{m^{\prime}, m}^{j}\left(\frac{\pi}{2}\right) d_{m^{\prime}, p}^{j}\left(\frac{\pi}{2}\right)\right], \\
= & \sum_{m=-j}^{j} \sum_{p=-j}^{j} C_{m}^{(j)} C_{p}^{(j) *} e^{-i \phi(m-p)} F_{m, p}\left(\frac{\pi}{2}\right),
\end{aligned}
$$

where the term in the square brackets is given by Eq. B13 Noting that $F_{m, p}\left(\lambda \rightarrow \frac{\pi}{2}\right)=(-1)^{2 j} \delta_{-m, p}$, we find for an arbitrary state

$$
\langle\hat{\Pi}\rangle=(-1)^{2 j} \sum_{m=-j}^{j} C_{-m}^{(j) *} C_{m}^{(j)} e^{-i 2 \phi m} .
$$

To aid in motivating the use of atomic parity as a detection observable let us first revisit the result for the most ideal of cases, that is, we analyze the measurement resolution obtained in a Ramsey interferometer when the state after the first $\pi / 2$ pulse is the atomic NOON state, given by

$$
\left|\psi_{\pi / 2}\right\rangle=\left|\psi_{\mathrm{N} 00 \mathrm{~N}}\right\rangle=\frac{1}{\sqrt{2}}\left(|j, j\rangle+e^{i \theta}|j,-j\rangle\right),
$$

including an arbitrary relative phase $\theta$. The representation of collections of two-level atoms in terms of the su(2) Lie alegbra, known as the Dicke model, is described in some detail in Appendix A. This can be expressed as a finite sum over all Dicke states as

$$
|\psi\rangle_{\mathrm{N} 00 \mathrm{~N}}=\sum_{m=-j}^{j} \Lambda_{m}^{(j)}|j, m\rangle,
$$

where the coefficients $\Lambda_{m}^{(j)}$ are given by

$$
\Lambda_{m}^{(j)}=\frac{1}{\sqrt{2}}\left(\delta_{m, j}+e^{i \theta} \delta_{m,-j}\right) .
$$


This state is of interest as it is the atomic analog of the optical N00N state $|\psi\rangle_{\mathrm{N} 00 \mathrm{~N}}=\frac{1}{\sqrt{2}}\left(|N, 0\rangle+e^{i \theta}|0, N\rangle\right)$ (to be discussed in greater depth), which yields the lower bound on phase uncertainty in quantum optical interferometry: the Heisenberg limit. This follows from the heuristic uncertainty relation between photon number and phase $\Delta \phi \Delta N \geq 1$, where the $\mathrm{N} 00 \mathrm{~N}$ state maximizes the uncertainty in photon number, that is, $\Delta N=N \rightarrow \Delta \phi \simeq 1 / \Delta N=1 / N$. The action of the interferometer is once again given by Eq. 147 where we assume some means of experimentally generating the atomic N00N state is employed prior to free evolution. Here we introduce the atomic parity operator as $\hat{\Pi}=\operatorname{Exp}\left[i \pi\left(\hat{J_{0}}-\hat{J_{3}}\right)\right]$, with respect to the number of atoms found in the ground state. We can likewise define atomic parity in terms of the number of atoms found in the excited state. The relationship between the two being $\left\langle\hat{\Pi}_{\text {Ground }}\right\rangle=(-1)^{2 j}\left\langle\hat{\Pi}_{\text {Excited }}\right\rangle$. This is sensible, since if $N$ is even and $2 n$ atoms are excited then $2 n^{\prime}$ atoms must also be in the ground state where $n, n^{\prime} \in \mathbb{Z}^{+}$. A similar argument can be made for $2 n+1$ exicted atoms. Likewise, if $N$ were odd and $2 n$ atoms are excited, then $2 n^{\prime}+1$ must be in the ground state, resulting in a sign difference in parity. With this, we calculate the expectation value of the parity operator using Eq. 149 to find

$$
\langle\hat{\Pi}\rangle_{\mathrm{NOON}}=(-1)^{N} \cos (N \phi+\theta),
$$

where $N=2 j$, the total number of atoms. Here, the relative phase $\theta$ acts as a phase shift of the interference pattern, which now oscillates at the enhanced rate $N \phi$. Finer measurement resolution, characterized by a narrowing of the peak of $\langle\hat{\Pi}\rangle$ at $\phi=0$ for increasing values of $N$, tends towards enhancing phase sensitivity in interferometric measurements. This state would serve as the ideal, as it is maximally entangled and yields the greatest measurement resolution for a given detection observable. The problem, however, is atomic N0ON states are incredibly difficult to make. We endeavor to find means of enhancing measurement resolution by considering parity-based measurements performed on both unentangled ensembles and initially entangled atomic systems.

\section{Unentangled ensemble of atoms}

Let us assume our state is initially prepared with all atoms in the ground state, that is $\mid$ in $\rangle=|j,-j\rangle$. The action of the Ramsey interferometer is to transform an initial state $\left|\psi^{\prime}\right\rangle$ by

$$
\begin{aligned}
\left|\Psi_{\mathrm{F}}\right\rangle & \left.=e^{-i \frac{\pi}{2} \hat{J}_{y}} e^{-i \phi \hat{J}_{z}} e^{-i \frac{\pi}{2} \hat{J}_{y}} \mid \text { in }\right\rangle \\
& =e^{-i \frac{\pi}{2} \hat{J}_{y}} e^{-i \phi \hat{J}_{z}}|\tau=-1, j\rangle,
\end{aligned}
$$

where $e^{-i \frac{\pi}{2} \hat{J}_{y}}|j,-j\rangle=|\tau=-1, j\rangle$ is the atomic coherent state in which all of the atoms in the ensemble are in the superposition state $\frac{1}{\sqrt{2}}(|g\rangle-|e\rangle)$, that is,

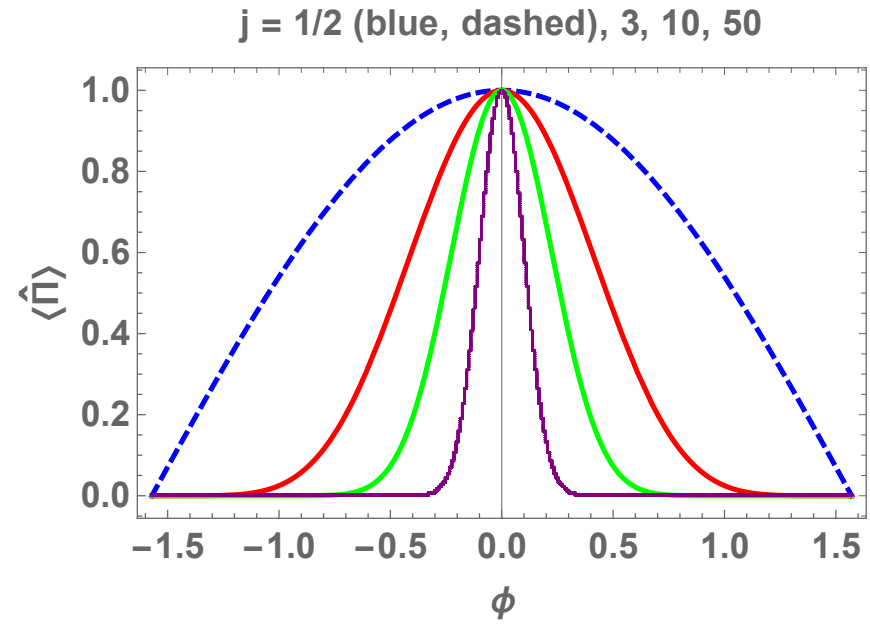

FIG. 17. Expectation value of the parity operator for an initial atomic coherent state for $j=1 / 2,3,10,50$. Note the peak becoming more narrow as the number of atoms is increased from $j=1 / 2$ (blue, dashed, broadest) to $j=50$ (purple, solid, narrowest).

$$
\begin{aligned}
|\tau=-1, j\rangle & =\frac{1}{2^{N / 2}}(|\mathrm{e}\rangle-|\mathrm{g}\rangle)^{\otimes N=2 j} \\
& =2^{-j} \sum_{m=-j}^{j}\left(\begin{array}{c}
2 j \\
j+m
\end{array}\right)^{1 / 2}(-1)^{j+m}|j, m\rangle .
\end{aligned}
$$

Let us recover the known result for an initial atomic coherent state. Taking the state coefficients of Eq. 155 and plugging them into Eq. 149 we find

$$
\begin{aligned}
\langle\hat{\Pi}\rangle_{\mathrm{ACS}} & =4^{-j} \sum_{m=-j}^{j}\left(\begin{array}{c}
2 j \\
j+m
\end{array}\right) e^{-i 2 \phi m}(-1)^{4 j}=d_{-j,-j}^{j}(2 \phi) \\
& =\cos ^{2 j} \phi=\cos ^{N} \phi
\end{aligned}
$$

This is plotted in Fig. 17 Unlike the case of simply taking the population difference, larger numbers of atoms yields greater measurement resolution, characterized by the narrowing of the peak about $\phi=0$. Comparing Eq. 156 result to Eq. 144 , we see that population difference measurement produces an interference cosine function raised to effectively $N=1$ photons, while the use of atomic parity instead has raised to the number of atoms $N$, leading to enhanced resolution at the origin as $\phi \rightarrow 0$. This can be demonstrated if we make the approximation $\cos ^{N} \phi \sim e^{-N \phi^{2} / 2}$ for small $\phi$ which is a Gaussian that narrows for increasing $N$. We remind the reader that while parity-based measurement yields greater resolution, this does not translate to better phase sensitivity beyond the SQL as the initial state is classical.

\section{Entangled atomic ensemble}

Now let us next consider the case where we act with the $\hat{J}_{z}$ operator after the first $\pi / 2$-pulse. The resulting state is both 
entangled at the atomic-state level and is known to exhibit spin-squeezing, which has been shown to reduce projection noise in high-precision population spectroscopy. In this case the initial state is transformed by

$$
\begin{aligned}
\left|\Psi_{\mathrm{F}}\right\rangle & \left.=e^{-i \frac{\pi}{2} \hat{J}_{y}} e^{-i \phi \hat{J}_{z}} \hat{J}_{z} e^{-i \frac{\pi}{2} \hat{J}_{y}} \mid \text { in }\right\rangle \\
& =e^{-i \frac{\pi}{2} \hat{J}_{y}} e^{-i \phi \hat{J}_{z}}|\tau=-1, j ; 1\rangle,
\end{aligned}
$$

where the state

$$
\begin{aligned}
\mid \tau= & -1, j ; 1\rangle \propto \hat{J}_{z}|\tau=-1, j\rangle \\
& =\left(2^{2 j-1} j\right)^{-1 / 2} \sum_{m=-j}^{j}\left(\begin{array}{c}
2 j \\
j+m
\end{array}\right)^{1 / 2}(-1)^{j+m} m|j, m\rangle .
\end{aligned}
$$

Once again, we use Eq. 149 to find the expectation value of the parity operator

$$
\begin{gathered}
\langle\hat{\Pi}\rangle_{\hat{J}_{z} \text {-op ACS }}=-\left(2^{2 j-1} j\right)^{-1} \sum_{m=-j}^{j}\left(\begin{array}{c}
2 j \\
j+m
\end{array}\right) m^{2} e^{-i 2 \phi m} \\
=-\frac{2}{\pi} \frac{\Gamma\left(j+\frac{1}{2}\right)}{\Gamma(j+2)} e^{-i 2 \phi}\left[{ }_{3} F_{2}\left(2,2,1-j ; 1, j+2 ;-e^{-i 2 \phi}\right)+\right. \\
\left.\quad+e^{i 4 \phi}{ }_{3} F_{2}\left(2,2,1-j ; 1, j+2 ;-e^{i 2 \phi}\right)\right]
\end{gathered}
$$

where ${ }_{3} F_{2}$ is the hypergeometric function given generally by Eq. B6 Simplifying for several different values of $j=N / 2$, yields

$$
\begin{aligned}
& j=1 / 2 \rightarrow\langle\hat{\Pi}\rangle_{\hat{J}_{z} \text { op ACS }}=-\cos \phi \\
& j=1 \rightarrow\langle\hat{\Pi}\rangle_{\hat{J}_{z} \text { op ACS }}=-\cos ^{2} \phi\left(2-\cos ^{-2} \phi\right) \\
& j=3 / 2 \rightarrow\langle\hat{\Pi}\rangle_{\hat{J}_{z} \text {-op ACS }}=-\cos ^{3} \phi\left(3-2 \cos ^{-2} \phi\right), \\
& j=2 \rightarrow\langle\hat{\Pi}\rangle_{\hat{J}_{z} \text { op ACS }}=-\cos ^{4} \phi\left(4-3 \cos ^{-2} \phi\right), \\
& j=5 / 2 \rightarrow\langle\hat{\Pi}\rangle_{\hat{J}_{z} \text { op ACS }}=-\cos ^{5} \phi\left(5-4 \cos ^{-2} \phi\right), \\
& j=3 \rightarrow\langle\hat{\Pi}\rangle_{\hat{J}_{z} \text {-op ACS }}=-\cos ^{6} \phi\left(6-5 \cos ^{-2} \phi\right),
\end{aligned}
$$

from which we can extrapolate for $N \geq 1$,

$$
\langle\hat{\Pi}\rangle_{\hat{J}_{z} \text {-op ACS }}^{(N)}=-\cos ^{N} \phi\left[N-(N-1) \cos ^{-2} \phi\right]
$$

We see that for the case of an initially entangled atomic ensemble, there is greater measurement resolution with increasing number of atoms over the initially unentangled ACS case, except for the case of $j=1 / 2(N=1)$ in which case both initial states yield the same resolution. This is demonstrated explicitly in Fig. 18 for the value of $N=2 j=6$, where we can

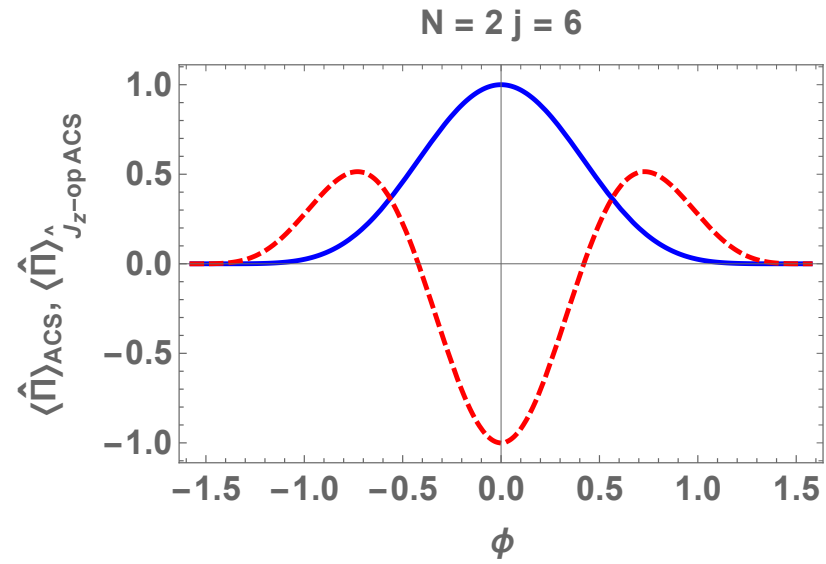

FIG. 18. A comparison between expectation values of the parity operator for the case of input ACS (blue, solid) and input $\hat{J}_{z}$-op ACS (red,dashed) for the same value of $N=2 j=6$. The resolution for the $\hat{J}_{z}$-op ACS is greater than the case of the ACS for all values of $N=1$.

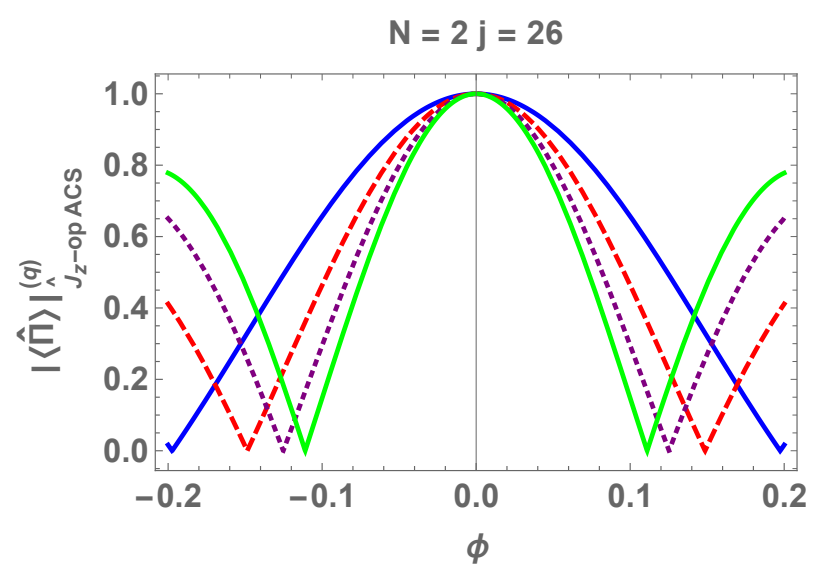

FIG. 19. The expectation value of the parity operator $|\langle\hat{\Pi}\rangle|$ for the case of an initial $\hat{J}_{z}^{q}$-operated ACS, for $N=2 j=26$. The curves in the figure above correspond to $q=1$ (blue, outer-solid), $q=2$ (red,dashed), $q=3$ (purple, dotted), $q=4$ (green, inner-solid). Note the increased resolution as the initial ensemble becomes more entangled.

see a narrowing of the peak about the phase origin for the same number of atoms when considering the initially-entangled case. This trend of enhanced resolution persists for $q>1$ applications of $\hat{J}_{z}$ prior to free evolution, which further entangles the initial atomic ensemble. For the general case of $q$ applications of $\hat{J}_{z}$ prior to free evolution, or $\mid$ in $\rangle \propto \hat{J}_{z}^{q}|\zeta=-1, j\rangle$, we can write the expectation value of the parity operator (unnormalized) as $\langle\hat{\Pi}\rangle_{\hat{J}_{z} \text {-op ACS }}^{(q)} \propto \sum_{m=-j}^{j}\left(\begin{array}{c}2 j \\ j+m\end{array}\right) m^{2 q} e^{-i 2 \phi m}$. Computations show greater resolutions obtained for the same (large) value of $j$ for increasing values of $q$, shown in Fig. 19 . 
VII. CONCLUSION

In this review article we discussed the prospect of using parity-based measurements in phase estimation. In the context of Ramsey spectroscopy, atomic parity is defined in terms of number of atoms found in the excited state while in the context of optical interferometry it is defined with regards to the average photon number in one output mode of the interferometer. We provide a brief discussion on the basics of phase estimation and demonstrate how parity-based detection saturates the quantum Cramér-Rao bound for path symmetric states in quantum optical interferometry, making parity the optimal observable for interferometric measurements. We highlight the use of parity-measurements for several different initial states comprising of both quantum and classical light and show agreement between the phase uncertainty when considering parity-based measurements and the quantum Cramér-Rao bound. For all quantum states considered, sub-SQL phase sensitivity is achieved when parity-based measurement is considered.

We also discuss the experimental efforts made towards measuring photon number parity in quantum optical interferometry using classical (coherent) light where the comparison is made to another dichotomic operator corresponding to "click"/"no click" detection. QND measures of atomic parity are also considered through coupling to both an ancillary atomic system that can be as arbitrarily small as a single atom as well as coupling to a light field prepared in a coherent state. We highlight that the latter case does not require a large third order non-linearity and in fact utilizes a readily available means of coupling.

We return to atomic spectroscopy in the closing section where the emphasis is placed on the enhanced measurement resolution obtained through parity-based measurement compared to the usual method of taking the population difference between the two internal degrees of freedom available to each atom. We show in the latter case that for arbitrary $N$ atom initial states, the Ramsey technique cannot yield super-resolved measurements while the former shows greater resolution for increasingly large numbers of atoms $N$. Of the cases discussed, we show an initially entangled atomic ensemble yields greater resolution for the same number of atoms than the case in which all atoms are initially unentangled and in the same atomic state.

\section{ACKNOWLEDGMENTS}

RJB acknowledges support from the National Research Council Research Associate Program (NRC RAP). CCG acknowledges support under AFRL Summer Faculty Fellowship Program (SFFP). PMA and CCG acknowledge support from the Air Force Office of Scientific Research (AFOSR). Any opinions, findings and conclusions or recommendations expressed in this material are those of the author(s) and do not necessarily reflect the views of the Air Force Research Laboratory
(AFRL).

Appendix A: A brief review of the $\mathrm{SU}(2)$ group

\section{The Dicke states}

In what follows, we prepare a brief review of the so-called Dicke states ${ }^{91}$ which provides a representation of a collection of $N$ two-level atoms in terms of the $\mathrm{SU}(2)$ "angular momentum" states $|j, m\rangle$ where $j=N / 2, m \in[-j, j]$ and where the internal basis states of the $i^{\text {th }}$ atom are $\left\{|e\rangle_{i},|g\rangle_{i}\right\}$. The quantity $j$ in this context is sometimes called the cooperation parameter. Only states symmetric with respect to the permutations of the $N$ atoms come into play in the cases considered here. That is, the direct product of the spaces of the two-level atoms, as given by $2_{1} \otimes 2_{2} \otimes 2_{3} \otimes \ldots \otimes 2_{N}$, has one symmetric and generally several anti-symmetric decompositions, but the anti-symmetric states are dynamically decoupled from the symmetric states via the selection rules. To demonstrate the construction of the symmetric states and connect them to the angular momentum states, we proceed by example where we use the ladder operators given in terms of the individual atoms as

$$
\hat{J}_{+}=\sum_{i=1}^{N}|e\rangle_{i}\left\langle g\left|, \quad \hat{J}_{-}=\sum_{i=1}^{N}\right| g\right\rangle_{i}\langle e|,
$$

and use the relations

$$
\hat{J}_{ \pm}|j, m\rangle=\sqrt{j(j+1)-m(m \pm 1)}|j, m \pm 1\rangle
$$

acting on the collective angular momentum states. In actuality, multiple applications of the raising operator are used starting from the state in which all atoms are in their ground state.

For two atoms we have $j=N / 2=1$; the state in which both atoms are in their ground state is state in which $m=-j=-1$ such that $|j=1, m=-1\rangle=|g\rangle_{1}|g\rangle_{2}$, or more simply

$$
|1,-1\rangle=|g\rangle_{1}|g\rangle_{2}
$$

Applying $\hat{J}_{+}$to both sides and using the above relations we find

$$
|1,0\rangle=\frac{1}{\sqrt{2}}\left(|e\rangle_{1}|g\rangle_{2}+|g\rangle_{1}|e\rangle_{2}\right)
$$

and applying it again yields

$$
|1,1\rangle=|e\rangle_{1}|e\rangle_{2}
$$

We could have obtained the same set of states had we started with the state $|1,1\rangle=|e\rangle_{1}|e\rangle_{2}$ and laddered down to $|1,-1\rangle=$ 
$|g\rangle_{1}|g\rangle_{2}$ through consecutive applications of the $\hat{J}_{-}$. These are the symmetric states, or Dicke states, for the case of two atoms. Likewise one can see that for three atoms $j=N / 2=$ $3 / 2$ and the Dicke states are

$$
\begin{aligned}
& \left|\frac{3}{2},-\frac{3}{2}\right\rangle=|g\rangle_{1}|g\rangle_{2}|g\rangle_{3}, \\
& \left|\frac{3}{2},-\frac{1}{2}\right\rangle=\frac{1}{\sqrt{3}}\left(|e\rangle_{1}|g\rangle_{2}|g\rangle_{3}+|g\rangle_{1}|e\rangle_{2}|g\rangle_{3}+|g\rangle_{1}|g\rangle_{2}|e\rangle_{3}\right), \\
& \left|\frac{3}{2}, \frac{1}{2}\right\rangle=\frac{1}{\sqrt{3}}\left(|e\rangle_{1}|e\rangle_{2}|g\rangle_{3}+|g\rangle_{1}|e\rangle_{2}|e\rangle_{3}+|e\rangle_{1}|g\rangle_{2}|e\rangle_{3}\right), \\
& \left|\frac{3}{2}, \frac{3}{2}\right\rangle=|e\rangle_{1}|e\rangle_{2}|e\rangle_{3} .
\end{aligned}
$$

The process can be continued for any number of atoms. Note that the extremal states $|j, \pm j\rangle$ are (separable) product states whereas all other states $m \neq \pm j$ are entangled at the atomic level.

\section{a. The atomic coherent state}

Two definitions of the atomic coherent state have been given in the literature: one by Radcliffe ${ }^{92}$ and another by Arrechi $e t$ $a l{ }^{10]}$ The definitions invlove the action of the SU(2) "displacement" operator $R(\theta, \varphi)=e^{\zeta \hat{J}_{+}-\zeta^{*} \hat{J}_{-}}$on the extremal states $j, \hat{ \pm} j$ where $\zeta=\frac{\theta}{2} e^{-i \varphi}$. The angles $\theta, \varphi$ are the usual angles parameterizing the Bloch sphere with $0 \leq \theta \leq \pi$ and $0 \leq \varphi \leq 2 \pi$ where we follow Arrechi et al. in taking $\theta=0$ to be the south pole of the sphere. This operator acts on the $|j, j\rangle$ state in the case of the Radcliffe definition and on the $|j,-j\rangle$ state in the case of Arrechi et al. For these two separate definitions, we require two different orderings of the "disentanglement" expressions of the operator $R(\theta, \varphi)$. These are

$$
e^{\zeta \hat{J}_{+}-\zeta^{*} \hat{J}_{-}}=\left\{\begin{array}{l}
e^{\tau \hat{J}_{+}} e^{\ln \left(1+|\tau|^{2}\right) \hat{J}_{z}} e^{-\tau^{*} \hat{J}_{-}}, \\
e^{-\tau^{*} \hat{J}_{-}} e^{-\ln \left(1+|\tau|^{2}\right) \hat{J}_{z}} e^{\tau \hat{J}_{+}},
\end{array}\right.
$$

where $\tau=e^{-i \varphi} \tan \frac{\theta}{2}$. In starting with the state $|j,-j\rangle$ we use the upper expression on the right hand side of Eq. A7]to arrive at

$$
\begin{aligned}
|\tau, j\rangle & =\left(1+|\tau|^{2}\right)^{-j} e^{\tau{\hat{J_{+}}}_{+}}|j,-j\rangle \\
& =\left(1+|\tau|^{2}\right)^{-j} \sum_{m=-j}^{j}\left(\begin{array}{c}
2 j \\
j+m
\end{array}\right)^{1 / 2} \tau^{j+m}|j, m\rangle,
\end{aligned}
$$

and using the bottom line of the right hand side of Eq. A7 we obtain

$$
\begin{aligned}
\left|-\tau^{*}, j\right\rangle & =\left(1+|\tau|^{2}\right)^{-j} e^{-\tau^{*} \hat{J}_{+}}|j,-j\rangle \\
& =\left(1+|\tau|^{2}\right)^{-j} \sum_{m=-j}^{j}\left(\begin{array}{c}
2 j \\
j+m
\end{array}\right)^{1 / 2}\left(-\tau^{*}\right)^{j+m}|j, m\rangle
\end{aligned}
$$

Note that under the replacement $\tau \leftrightarrow-\tau^{*}$, these states are the same.

For the special case used throughout Sections $\Pi$ and $\mathrm{VI}$ where the rotation operator $e^{-i \frac{\pi}{2} \hat{J}_{y}}$ acts on the states $|j, \pm j\rangle$ states, we can set

$$
R(\theta, \varphi)=e^{\zeta \hat{J}_{+}-\zeta^{*} \hat{J}_{-}} \equiv e^{-i \frac{\pi}{2} \hat{J}_{y}}
$$

informing the value $\zeta=-\pi / 4$ and consequently the angles $\theta=\pi / 2$ and $\varphi=\pi$. Thus we have $\tau=-1$ and $-\tau^{*}=1$ which, for example, is demonstrated in Eq. 15. More simply, one can insert a full set of states to find

$$
\begin{aligned}
e^{-i \frac{\pi}{2} \hat{J}_{y}}|j, \pm j\rangle & =\sum_{m=-j}^{j}|j, m\rangle\left\langle j, m\left|e^{-i \frac{\pi}{2}}\right| j, \pm j\right\rangle \\
& =\sum_{m=-j}^{j} d_{m, \pm j}^{j}\left(\frac{\pi}{2}\right)|j, m\rangle \\
& =\left(1+|\tau|^{2}\right)^{-j} \sum_{m=-j}^{j}\left(\begin{array}{c}
2 j \\
j+m
\end{array}\right)( \pm 1)^{j+m}|j, m\rangle
\end{aligned}
$$

where the matrix elements $d_{m, \pm j}^{j}\left(\frac{\pi}{2}\right)$ are the Wigner- $d$ matrix elements found in Rose ${ }^{93}$ and given for this special case in Eq. B11.

\section{The Schwinger realization of SU(2)}

Consider a two mode field with creation and annihilation operators satisfying the usual boson commutation relations $\left[\hat{a}_{i}, \hat{a}_{j}\right]=\left[\hat{a}_{i}^{\dagger}, \hat{a}_{j}^{\dagger}\right]=0$ and $\left[\hat{a}_{i}, \hat{a}_{j}^{\dagger}\right]=\delta_{i, j}$. One can introduce the Hermitian operators

$$
\begin{aligned}
& \hat{J}_{x}=\frac{1}{2}\left(\hat{a}_{1}^{\dagger} \hat{a}_{2}+\hat{a}_{2}^{\dagger} \hat{a}_{1}\right), \\
& \hat{J}_{y}=-\frac{i}{2}\left(\hat{a}_{1}^{\dagger} \hat{a}_{2}-\hat{a}_{2}^{\dagger} \hat{a}_{1}\right), \\
& \hat{J}_{z}=\frac{1}{2}\left(\hat{a}_{1}^{\dagger} \hat{a}_{1}-\hat{a}_{2}^{\dagger} \hat{a}_{2}\right),
\end{aligned}
$$

and $\hat{N}=\frac{1}{2}\left(\hat{a}_{1}^{\dagger} \hat{a}_{1}+\hat{a}_{2}^{\dagger} \hat{a}_{2}\right)$, satisfying the commutation relations of the Lie algebra of $\mathrm{SU}(2)$ :

$$
\left[\hat{J_{i}}, \hat{J_{j}}\right]=i \hat{J}_{k} \varepsilon_{i, j, k}
$$

The Casimir invariant for the group can be found to be of the form $\hat{J}^{2}=\hat{J}_{x}^{2}+\hat{J}_{y}^{2}+\hat{J}_{z}^{2}=\frac{\hat{N}}{2}\left(\frac{\hat{N}}{2}+1\right)$. Note that $\hat{N}$ commutes will all operators in Eq. A12 One can also define the operator $\hat{J_{0}}=\frac{1}{2} \hat{N}$ such that $\hat{J}_{0}|j, m\rangle=j|j, m\rangle$. It is also useful to recall the action of the angular momentum operatos $\hat{J}_{i}$ on the states $|j, m\rangle$ : 


$$
\begin{aligned}
\hat{J}^{2}|j, m\rangle & =j(j+1)|j, m\rangle \\
\hat{J}_{z}|j, m\rangle & =m|j, m\rangle \\
\hat{J}_{ \pm}|j, m\rangle & =\sqrt{j(j+1)-m(m \pm 1)}|j, m \pm 1\rangle
\end{aligned}
$$

where the ladder operators can be written as $\hat{J}_{ \pm}=\hat{J}_{x} \pm i \hat{J}_{y}$. For bosons, we can translate between the Dicke (angular momentum) states $|j, m\rangle^{(a, b)}$ and Fock states

A beam splitter transforms the boson operators associated with each input mode according to the scattering matrix for the device, that is

$$
\overrightarrow{\hat{a}}_{\text {out }}=\hat{U} \overrightarrow{\hat{a}}_{\text {in }} \rightarrow\left(\begin{array}{l}
\hat{a}_{1} \\
\hat{a}_{2}
\end{array}\right)_{\text {out }}=\left(\begin{array}{ll}
U_{11} & U_{12} \\
U_{21} & U_{22}
\end{array}\right)\left(\begin{array}{l}
\hat{a}_{1} \\
\hat{a}_{2}
\end{array}\right)_{\text {in }} .
$$

Note that since the boson creation and annihilation must satisfy the commutation relations both before and after beamsplitting, the matrix $\hat{U}$ must be unitary. Let us see how this transforms the operators of $\mathrm{SU}(2), \vec{J}=\left(\hat{J}_{x}, \hat{J}_{y}, \hat{J}_{z}\right)$. Consider the $\hat{J}_{x}$-type beam splitter scattering matrix,

$$
\hat{U}=\left(\begin{array}{cc}
\cos \frac{\theta}{2} & -i \sin \frac{\theta}{2} \\
-i \sin \frac{\theta}{2} & \cos \frac{\theta}{2}
\end{array}\right)=\left(\begin{array}{cc}
t & -i r \\
-i r & t
\end{array}\right)
$$

which corresponds to a beam splitter with transmittance and reflectivity $T=\cos ^{2} \frac{\theta}{2}$ and $R=\sin ^{2} \frac{\theta}{2}$, respectively. For this scattering matrix, $\vec{J}$ transforms to

$$
\begin{aligned}
\left(\begin{array}{l}
\hat{J_{x}} \\
\hat{J}_{y} \\
\hat{J}_{z}
\end{array}\right)_{\text {out }} & =\left(\begin{array}{ccc}
1 & 0 & 0 \\
0 & \cos \theta & -\sin \theta \\
0 & \sin \theta & \cos \theta
\end{array}\right)\left(\begin{array}{l}
\hat{J}_{x} \\
\hat{J}_{y} \\
\hat{J}_{z}
\end{array}\right)_{\text {in }} \\
& =e^{i \theta \hat{J}_{x}}\left(\begin{array}{l}
\hat{J}_{x} \\
\hat{J}_{y} \\
\hat{J}_{z}
\end{array}\right)_{\text {in }} e^{-i \theta \hat{J}_{x}},
\end{aligned}
$$

which amounts to a rotation about the fictitious $x$-axis. Note that the last line of Eq. A17 can be verified via the use of the Baker-Hausdorff identity

$$
e^{\tau \hat{A}} \hat{B} e^{-\tau \hat{A}}=\hat{B}+\tau[\hat{A}, \hat{B}]+\frac{1}{2} \tau^{2}[\hat{A},[\hat{A}, \hat{B}]]+\ldots
$$

Working in the Schrödinger picture, the action of the beam splitter corresponds to a transformation of the initial state given by

$$
\left.\mid \text { out, } \mathrm{BS}\rangle=e^{-i \theta \hat{J}_{x}} \mid \text { in }\right\rangle .
$$

We can also express a two-mode state in the Fock basis in terms of the basis states of SU(2) (angular momentum states) using Eq. A12, yielding

$$
\begin{gathered}
\hat{J}_{z}|j, m\rangle=m|j, m\rangle \rightarrow \hat{J}_{z}\left|n, n^{\prime}\right\rangle_{1,2}=\frac{n-n^{\prime}}{2}\left|n, n^{\prime}\right\rangle_{1,2}, \\
\hat{J}^{2}|j, m\rangle=j(j+1)|j, m\rangle \rightarrow \hat{J}^{2}\left|n, n^{\prime}\right\rangle_{1,2}=\frac{n+n^{\prime}}{2}\left(\frac{n+n^{\prime}}{2}+1\right),
\end{gathered}
$$

which informs us that $\left|n, n^{\prime}\right\rangle_{1,2} \rightarrow|j, m\rangle$ where the values of $j$ and $m$ are given by $j=\frac{n+n^{\prime}}{2}$ and $m=\frac{n-n^{\prime}}{2}$. Inversely $|j, m\rangle \rightarrow$ $\left|n, n^{\prime}\right\rangle_{1,2}$ where $n=j+m$ and $n^{\prime}=j-m$ with $n+n^{\prime}=2 j$ and $m \in\{-j, \ldots, j\}$. Lastly, the action of the su(2) ladder operators in Fock basis is

$$
\begin{aligned}
& \hat{J_{ \pm}}|j, m\rangle=\sqrt{j(j+1)-m(m \pm 1)}|j, m \pm 1\rangle \rightarrow \\
& \rightarrow \hat{J}_{ \pm}\left|n, n^{\prime}\right\rangle_{1,2}= \\
& \frac{1}{2} \sqrt{\left(n+n^{\prime}\right)\left(n+n^{\prime}+2\right)-\left(n-n^{\prime}\right)\left(n-n^{\prime} \pm 2\right)} \times \\
& \quad \times\left|n \pm 1, n^{\prime} \mp 1\right\rangle_{1,2} .
\end{aligned}
$$

The connection between two-mode boson fields and the "angular momentum" states of su(2) is now complete.

\section{Beam splitter transformations}

Throughout this paper, several different beam splitter transformations are utilized in conjunction with optical interferometry. Here we endeavor to show how these particular transformations affect the corresponding boson mode operators. First, let us reconsider the scattering matrix of Eq. A16 corresponding to a $\hat{J}_{x}$-type beam splitter such that $\mid$ out, $\left.\mathrm{BS}\right\rangle=e^{-i \theta \hat{J}_{x}} \mid$ in $\rangle$ as per Eq. A19 For the choices of angle $\theta= \pm \pi / 2$, both corresponding to a $50: 50$ beam splitter but parameterized by different rotations about the fictitious $x$-axis, the mode operators transform according to

$$
\left(\begin{array}{l}
\hat{a}_{0}^{\prime} \\
\hat{a}_{1}^{\prime}
\end{array}\right)=\frac{1}{\sqrt{2}}\left(\begin{array}{cc}
1 & \mp i \\
\mp i & 1
\end{array}\right)\left(\begin{array}{l}
\hat{a}_{0} \\
\hat{a}_{1}
\end{array}\right)
$$

Let us consider two modes of an optical field labeled $a$ - and $b$ - modes for which operators $\left\{\hat{a}_{0}, \hat{a}_{0}^{\prime}\right\}$ act on the $a$-mode and $\left\{\hat{a}_{1}, \hat{a}_{1}^{\prime}\right\}$ on the $b$-mode. For $\theta= \pm \pi / 2$ and using Eq. A22 we can write

$$
\begin{array}{ll}
\hat{a}_{0}^{\prime}=\frac{1}{\sqrt{2}}\left(\hat{a}_{0} \mp i \hat{a}_{1}\right), & \hat{a}_{0}=\frac{1}{\sqrt{2}}\left(\hat{a}_{0}^{\prime} \pm i \hat{a}_{1}^{\prime}\right), \\
\hat{a}_{1}^{\prime}=\frac{1}{\sqrt{2}}\left(\hat{a}_{1} \mp i \hat{a}_{0}\right), & \hat{a}_{1}=\frac{1}{\sqrt{2}}\left(\hat{a}_{1}^{\prime} \pm i \hat{a}_{0}^{\prime}\right) .
\end{array}
$$

Direct substitution of the mode operators in Eq. A23 yields for the displacement operators 


$$
\begin{aligned}
\hat{D}_{a}(\alpha)=e^{\alpha \hat{a}_{0}^{\dagger}-\alpha^{*} \hat{a}_{0}} & \rightarrow e^{\frac{\alpha}{\sqrt{2}}\left(\hat{a}_{0}^{\dagger \prime} \mp i \hat{a}_{1}^{\dagger \prime}\right)-\frac{\alpha^{*}}{\sqrt{2}}\left(\hat{a}_{0}^{\prime} \pm i \hat{a}_{1}^{\prime}\right)} \\
& \rightarrow e^{\frac{\alpha}{\sqrt{2}} \hat{a}_{0}^{\dagger \prime}-\frac{\alpha^{*}}{\sqrt{2}} \hat{a}_{0}^{\prime}} e^{\mp \frac{i \alpha}{\sqrt{2}} \hat{a}_{1}^{\dagger \prime} \mp \frac{i \alpha^{*}}{\sqrt{2}} \hat{a}_{1}^{\prime}} \\
& \rightarrow \hat{D}_{a}\left(\frac{\alpha}{\sqrt{2}}\right) \hat{D}_{b}\left(\mp \frac{i \alpha}{\sqrt{2}}\right) \\
\hat{D}_{b}(\beta)=e^{\beta \hat{a}_{1}^{\dagger}-\beta^{*} \hat{a}_{1}} & \rightarrow e^{\frac{\beta}{\sqrt{2}}\left(\hat{a}_{1}^{\dagger \prime} \mp i \hat{a}_{0}^{\dagger \prime}\right)-\frac{\beta^{*}}{\sqrt{2}}\left(\hat{a}_{1}^{\prime} \pm i \hat{a}_{0}^{\prime}\right)} \\
& \rightarrow e^{\mp \frac{i \beta}{\sqrt{2}} \hat{a}_{0}^{\dagger \prime} \mp \frac{i \beta^{*}}{\sqrt{2}} \hat{a}_{0}^{\prime}} e^{\frac{\beta}{\sqrt{2}} \hat{a}_{1}^{\dagger \prime}-\frac{\beta^{*}}{\sqrt{2}} \hat{a}_{1}^{\prime}} \\
& \rightarrow \hat{D}_{a}\left(\mp \frac{i \beta}{\sqrt{2}}\right) \hat{D}_{b}\left(\frac{\beta}{\sqrt{2}}\right),
\end{aligned}
$$

or noting the mode operators transform as $e^{\mp i \frac{\pi}{2} \hat{J}_{x}} \hat{a}_{0} e^{ \pm i \frac{\pi}{2} \hat{J}_{x}}=$ $\frac{1}{\sqrt{2}}\left(\hat{a}_{0} \pm i \hat{a}_{1}\right)$ and $e^{\mp i \frac{\pi}{2} \hat{J}_{x}} \hat{a}_{1} e^{ \pm i \frac{\pi}{2} \hat{J}_{x}}=\frac{1}{\sqrt{2}}\left(\hat{a}_{1} \pm i \hat{a}_{0}\right)$, we can write more succinctly

$$
\begin{aligned}
& e^{\mp i \frac{\pi}{2} \hat{J}_{x}} \hat{D}_{a}(\alpha) e^{ \pm i \frac{\pi}{2} \hat{J}_{x}}=\hat{D}_{a}\left(\frac{\alpha}{\sqrt{2}}\right) \hat{D}_{b}\left(\mp \frac{i \alpha}{\sqrt{2}}\right) \\
& e^{\mp i \frac{\pi}{2} \hat{J}_{x}} \hat{D}_{b}(\beta) e^{ \pm i \frac{\pi}{2} \hat{J}_{x}}=\hat{D}_{a}\left(\mp \frac{i \beta}{\sqrt{2}}\right) \hat{D}_{b}\left(\frac{\beta}{\sqrt{2}}\right)
\end{aligned}
$$

For the case in which coherent light is incident on a beamsplitter from each input port, $\mid$ in $\rangle=|\alpha\rangle_{a}|\beta\rangle_{b}$, the resulting output state is straight forward to work out from the discussion above ${ }^{94}$ :

$$
\begin{aligned}
\mid \text { out, BS }\rangle & \left.=e^{-i \theta \hat{J}_{x}} \mid \text { in }\right\rangle \stackrel{\theta \rightarrow \pm \frac{\pi}{2}}{=} e^{\mp i \frac{\pi}{2} \hat{J}_{x}}|\alpha\rangle_{a}|\beta\rangle_{b} \\
& =\left|\frac{1}{\sqrt{2}}(\alpha \mp i \beta)\right\rangle_{a}\left|\frac{1}{\sqrt{2}}(\beta \mp i \alpha)\right\rangle_{b} .
\end{aligned}
$$

The resulting state is an equal-intensity distribution of light in each output mode, but where the reflected beam picks up a phase shift of $\pm \pi / 2$. For the case in which $\beta \rightarrow 0$, the initial state is $\mid$ in $\rangle=|\alpha\rangle_{a}|0\rangle_{b}$ and the output state is simply $\mid$ out $\rangle=e^{\mp i \frac{\pi}{2} \hat{J}_{x}} \mid$ in $\rangle=\left|\frac{\alpha}{\sqrt{2}}\right\rangle_{a}\left|\mp \frac{i \alpha}{\sqrt{2}}\right\rangle$.

For a $\hat{J_{y}}$-type beam splitter such that $\mid$ out $\rangle=e^{-i \theta \hat{J_{y}}} \mid$ in $\rangle$, the action of the scattering matrix transforms the mode operators according to

$$
\left(\begin{array}{l}
\hat{a}_{0}^{\prime} \\
\hat{a}_{1}^{\prime}
\end{array}\right)=\left(\begin{array}{cc}
\cos \frac{\theta}{2} & -\sin \frac{\theta}{2} \\
\sin \frac{\theta}{2} & \cos \frac{\theta}{2}
\end{array}\right)\left(\begin{array}{l}
\hat{a}_{0} \\
\hat{a}_{1}
\end{array}\right) \stackrel{\theta \rightarrow \pm \frac{\pi}{2}}{=} \frac{1}{\sqrt{2}}\left(\begin{array}{cc}
1 & \mp 1 \\
\pm 1 & 1
\end{array}\right)\left(\begin{array}{l}
\hat{a}_{0} \\
\hat{a}_{1}
\end{array}\right) .
$$

leading to

$$
\begin{array}{ll}
\hat{a}_{0}^{\prime}=\frac{1}{\sqrt{2}}\left(\hat{a}_{0} \mp \hat{a}_{1}\right), & \hat{a}_{0}=\frac{1}{\sqrt{2}}\left(\hat{a}_{0}^{\prime} \pm \hat{a}_{1}^{\prime}\right), \\
\hat{a}_{1}^{\prime}=\frac{1}{\sqrt{2}}\left(\hat{a}_{1} \pm \hat{a}_{0}\right), & \hat{a}_{1}=\frac{1}{\sqrt{2}}\left(\hat{a}_{1}^{\prime} \mp \hat{a}_{0}^{\prime}\right) .
\end{array}
$$

Direct substitution into the single-mode displacement operators yields

$$
\begin{aligned}
\hat{D}_{a}(\alpha)=e^{\alpha \hat{a}_{0}^{\dagger}-\alpha^{*} \hat{a}_{0}} & \rightarrow e^{\frac{\alpha}{\sqrt{2}}\left(\hat{a}_{0}^{\dagger \prime} \pm \hat{a}_{1}^{\dagger \prime}\right)-\frac{\alpha^{*}}{\sqrt{2}}\left(\hat{a}_{0}^{\prime} \pm \hat{a}_{1}^{\prime}\right)} \\
& \rightarrow e^{\frac{\alpha}{\sqrt{2}} \hat{a}_{0}^{\dagger \prime}-\frac{\alpha^{*}}{\sqrt{2}} \hat{a}_{0}^{\prime}} e^{ \pm \frac{\alpha}{\sqrt{2}} \hat{a}_{1}^{\dagger \prime} \mp \frac{\alpha^{*}}{\sqrt{2}} \hat{a}_{1}^{\prime}} \\
& \rightarrow \hat{D}_{a}\left(\frac{\alpha}{\sqrt{2}}\right) \hat{D}_{b}\left( \pm \frac{\alpha}{\sqrt{2}}\right), \\
\hat{D}_{b}(\beta)=e^{\beta \hat{a}_{1}^{\dagger}-\beta^{*} \hat{a}_{1}} & \rightarrow e^{\frac{\beta}{\sqrt{2}}\left(\hat{a}_{1}^{\dagger \prime} \mp \hat{a}_{0}^{\dagger \prime}\right)-\frac{\beta^{*}}{\sqrt{2}}\left(\hat{a}_{1}^{\prime} \mp \hat{a}_{0}^{\prime}\right)} \\
& \rightarrow e^{\frac{\beta}{\sqrt{2}} \hat{a}_{1}^{\dagger \prime}-\frac{\beta^{*}}{\sqrt{2}} \hat{a}_{1}^{\prime}} e^{\mp \frac{\beta}{\sqrt{2}} \hat{a}_{0}^{\dagger \prime} \pm \frac{\beta^{*}}{\sqrt{2}} \hat{a}_{0}^{\prime}} \\
& \rightarrow \hat{D}_{a}\left(\mp \frac{\beta}{\sqrt{2}}\right) \hat{D}_{b}\left(\frac{\beta}{\sqrt{2}}\right),
\end{aligned}
$$

Once again assuming coherent light initially in each port incident on a $50: 50$ beam splitter, the output state is given by

$$
\begin{aligned}
\mid \text { out, BS }\rangle & \left.=e^{-i \theta \hat{J_{y}}} \mid \text { in }\right\rangle \stackrel{\theta \rightarrow \pm \frac{\pi}{2}}{=} e^{\mp i \frac{\pi}{2} \hat{J}_{y}}|\alpha\rangle_{a}|\beta\rangle_{b} \\
& =\left|\frac{1}{\sqrt{2}}(\alpha \mp \beta)\right\rangle_{a}\left|\frac{1}{\sqrt{2}}(\beta \pm \alpha)\right\rangle_{b} .
\end{aligned}
$$

where once again the result is an equal-intensity distribution in both output modes. For $\beta \rightarrow 0$, the output state is given by $\mid$ out $\rangle=\left|\frac{\alpha}{\sqrt{2}}\right\rangle_{a}\left| \pm \frac{\alpha}{\sqrt{2}}\right\rangle$, where the choice of $\theta=-\pi / 2$ results in a phase shift of $\pi$ in the reflected beam.

\section{Appendix B: The Wigner- $d$ matrix elements}

\section{Defined}

Here we provide a brief discussion on the matrix elements of an arbitrary rotation specified by an axis of rotation $\hat{\boldsymbol{n}}$ and angle of rotation $\phi$. The matrix elements, with $\hbar \rightarrow 1$ for convenience, are

$$
\mathscr{D}_{m^{\prime}, m}^{j}(R)=\left\langle j, m^{\prime}\left|e^{-i \phi \boldsymbol{J} \cdot \hat{\boldsymbol{n}}}\right| j, m\right\rangle
$$

Since the rotation operator commutes with the $\hat{J}^{2}$ operator, a rotation cannot change the $j$ value of a state. The $(2 j+1) \times(2 j+1)$ matrix formed by $\mathscr{D}_{m^{\prime}, m}^{j}(R)$ is referred to as the $(2 j+1)$-dimensional irreducible representation of the rotation operator $\mathscr{D}(R)$. We now consider the matrix realization of the Euler Rotation,

$$
\begin{aligned}
\mathscr{D}_{m^{\prime}, m}^{j}(\alpha, \beta, \gamma) & =\left\langle j, m^{\prime}\left|R_{z_{f}}(\alpha) R_{y_{f}}(\beta) R_{z_{f}}(\gamma)\right| j, m\right\rangle \\
& =\left\langle j, m^{\prime}\left|e^{-i \alpha \hat{J}_{z}} e^{-i \beta \hat{J}_{y}} e^{-i \gamma \hat{J}_{z}}\right| j, m\right\rangle
\end{aligned}
$$

These matrix elements are referred to as the Wigner- $D$ matrix elements. Notice that the first and last rotation only add a phase factor to the expression, thus making only the rotation 
about the fixed $y$-axis the only non-trival part of the matrix. For this reason, the Wigner- $D$ matrix elements are written in terms of a new matrix

$$
\begin{aligned}
\mathscr{D}_{m^{\prime}, m}^{j}(\alpha, \beta, \gamma) & =\left\langle j, m^{\prime}\left|e^{-i \alpha \hat{J}_{z}} e^{-i \beta \hat{J}_{y}} e^{-i \gamma \hat{J}_{z}}\right| j, m\right\rangle \\
& =e^{-i\left(m^{\prime} \alpha+m \gamma\right)}\left\langle j, m^{\prime}\left|e^{-i \beta \hat{J}_{y}}\right| j, m\right\rangle \\
& =e^{-i\left(m^{\prime} \alpha+m \gamma\right)} d_{m^{\prime}, m}^{j}(\beta)
\end{aligned}
$$

where the matrix elements $d_{m^{\prime}, m}^{j}(\beta)=\left\langle j, m^{\prime}\left|e^{-i \beta \hat{J}_{y}}\right| j, m\right\rangle$ are known as the Wigner- $d$ matrix elements and are given by

$$
\begin{aligned}
d_{m^{\prime}, m}^{j}(\beta) & =\left(\frac{(j-m) !\left(j+m^{\prime}\right) !}{(j+m) !\left(j-m^{\prime}\right) !}\right)^{1 / 2} \times \\
& \times \frac{(-1)^{m^{\prime}-m} \cos ^{2 j+m-m^{\prime}}\left(\frac{\beta}{2}\right) \sin ^{m^{\prime}-m}\left(\frac{\beta}{2}\right)}{\left(m^{\prime}-m\right) !} \times \\
& \times{ }_{2} F_{1}\left(m^{\prime}-j,-m-j ; m^{\prime}-m+1 ;-\tan ^{2}\left(\frac{\beta}{2}\right)\right),
\end{aligned}
$$

with the property

$$
d_{m^{\prime} m}^{j}(\beta)= \begin{cases}d_{m^{\prime}, m}^{j}(\beta) & m^{\prime} \geq m \\ d_{m, m^{\prime}}^{j}(-\beta) & m^{\prime}<m\end{cases}
$$

and where ${ }_{2} F_{1}(a, b ; c ; z)$ is the hypergeometric function defined formally by

$$
{ }_{p} F_{q}\left(a_{1}, \ldots, a_{p} ; b_{1}, \ldots, b_{q} ; z\right)=\sum_{n=0}^{\infty} \frac{\left(a_{1}\right)_{n} \ldots\left(a_{p}\right)_{n}}{\left(b_{1}\right)_{n} \ldots\left(b_{q}\right)_{n}} \frac{z^{n}}{n !}
$$

The Pochhammer symbol above is used to express $(x)_{n}=$ $x(x+1)(x+2) \ldots(x+n-1)=\Gamma(x+n) / \Gamma(x)$ for $n \geq 1$ with the Gamma (generalized factorial) function $\Gamma(x)=(x-1)$ !. It is worth noting that in our interferometric calculations, we naturally end up with an expression that depends on the Wigner- $d$ matrix elements. However, when simply dealing with a single $\hat{J_{x}}$ beam splitter of angle $\theta$, one encounters the matrix elements $\left\langle j, m^{\prime}\left|e^{-i \theta \hat{J}_{x}}\right| j, m\right\rangle$. This can be simplified using the relations to

$$
\begin{aligned}
\left\langle j, m^{\prime}\left|e^{-i \theta \hat{J}_{x}}\right| j, m\right\rangle & =\left\langle j, m^{\prime}\left|e^{i \frac{\pi}{2} \hat{J}_{z}} e^{-i \theta \hat{J}_{y}} e^{-i \frac{\pi}{2} \hat{J}_{z}}\right| j, m\right\rangle \\
& =\mathscr{D}_{m^{\prime}, m}^{j}\left(-\frac{\pi}{2}, \theta, \frac{\pi}{2}\right) \\
& =i^{m^{\prime}-m} d_{m^{\prime}, m}^{j}(\theta) .
\end{aligned}
$$

In our closing section we state a handful of useful identities used throughout this paper.

\section{Useful identities}

Within the body of this review article, several simplified forms of the Wigner- $d$ matrix are used. The cases relevant to the material herein are listed below:

$$
\begin{gathered}
d_{-j,-j}^{j}(\beta)=d_{j, j}^{j}(\beta)=\cos ^{2 j}\left(\frac{\beta}{2}\right), \\
d_{j,-j}^{j}(\beta)=(-1)^{2 j} d_{-j, j}^{j}(\beta)=(-1)^{2 j} \sin ^{2 j}\left(\frac{\beta}{2}\right),
\end{gathered}
$$

where we have used the identity

$$
d_{m^{\prime}, m}^{j}(\beta)=(-1)^{m^{\prime}-m} d_{m, m^{\prime}}^{j}(\beta) .
$$

We have also used the simplified form

$$
d_{m,-j}^{j}\left(\frac{\pi}{2}\right)=2^{-j}\left(\begin{array}{c}
2 j \\
j+m
\end{array}\right)^{1 / 2}(-1)^{j+m}=(-1)^{j+m} d_{m, j}^{j}\left(\frac{\pi}{2}\right),
$$

in our derivations pertaining to Ramsey spectroscopy. Through applications of the standard relations involving the Wigner- $d$ rotation elements 95 , it can be shown

$\sum_{m^{\prime}=-j}^{j}(-1)^{j-m^{\prime}} d_{j-n, m^{\prime}}^{j}(-\beta) d_{m^{\prime}, j-n}^{j}(\beta)=(-1)^{n} d_{j-n, j-n}^{j}(2 \beta)$

so that in particular for $n=0$ we find $d_{j, j}^{j}(2 \phi)=\cos ^{2 j} \phi$. This identity is used in Eq. 98 in the derivation of the expectation value of the parity operator $\left\langle\hat{\Pi}_{b}\right\rangle$ for the input state $|\alpha\rangle_{a} \otimes|N\rangle_{b}$.

The following functions are defined in conjunction with the Ramsey spectroscopy derivations of Eqs. 156, 144 and 145 . respectively:

$$
\begin{aligned}
F_{m, p}(\lambda) & =\sum_{m^{\prime}=-j}^{j}(-1)^{j-m^{\prime}} d_{m^{\prime}, m}^{j}(\lambda) d_{m^{\prime}, p}^{j}(\lambda) \\
& =(-1)^{2 j} \sum_{m^{\prime}} d_{m^{\prime},-m}^{j}(\pi-\lambda) d_{m^{\prime}, p}^{j}(\lambda) \\
& =(-1)^{2 j} \sum_{m^{\prime}} d_{p, m^{\prime}}^{j}(-\lambda) d_{m^{\prime},-m}^{j}(\pi-\lambda) \\
& =(-1)^{2 j} d_{p,-m}^{j}(\pi-2 \lambda) .
\end{aligned}
$$

$$
\begin{aligned}
\Gamma_{m, p}(\lambda) & =\sum_{m^{\prime}=-j}^{j} m^{\prime} d_{m^{\prime}, p}^{j}(\lambda) d_{m^{\prime}, m}^{j}(\lambda) \\
& =\sum_{m^{\prime}=-j}^{j} m^{\prime} d_{m^{\prime}, p}^{j}(\lambda) d_{m^{\prime}, m}^{j}(\lambda)\left(\delta_{p, m+1}+\delta_{p+1, m}+\delta_{p, m}\right)
\end{aligned}
$$




$$
\begin{aligned}
\Lambda_{m, p}(\lambda)= & \sum_{m^{\prime}=-j}^{j} m^{\prime 2} d_{m^{\prime}, p}^{j}(\lambda) d_{m^{\prime}, m}^{j}(\lambda) \\
= & \sum_{m^{\prime}=-j}^{j} m^{\prime 2} d_{m^{\prime}, p}^{j}(\lambda) d_{m^{\prime}, m}^{j}(\lambda) \times \\
& \times\left(\delta_{p, m+2}+\delta_{p+2, m}+\delta_{p, m+1}+\delta_{p+1, m}+\delta_{p, m}\right)
\end{aligned}
$$

${ }^{1}$ E. Wigner, "On the quantum correction for thermodynamic equilibrium," Phys. Rev. 40, 749-759 (1932).

${ }^{2}$ K. E. Chaill and R. J. Glauber, "Density operators and quasiprobability distributions," Phys. Rev. 177, 5 (1969).

${ }^{3}$ A. Royer, "Wigner function as the expectation value of a parity operator," Phys. Rev. A 15, 2 (1977).

${ }^{4} \mathrm{U}$. Leonhardt, Measuring the Quantum State of Light (Cambridge University Press, Cambridge, UK., 1997).

${ }^{5} \mathrm{~K}$. Banaszek and K. Wódkiewicz, "Nonlocality of the Einstein-PodolskyRosen state in the Wigner representation," Phys. Rev. A 58, 6 (1998).

${ }^{6} \mathrm{~L}$. Barsotti, J. Harms, and R. Schnabel, "Squeezed vacuum states of light for gravitational wave detectors," Rep. Prog. Phys. 82, 016905 (2019).

${ }^{7}$ J. J. Bollinger, W. M. Itano, D. J. Wineland, and D. J. Heinzen, "Optimal frequency measurements with maximally correlated states," Phys. Lett. A 54, R4649 (1996).

${ }^{8}$ B. Yurke, S. L. McCall, and J. R. Klauder, "SU(2) and SU(1,1) interferometers," Phys. Rev. A 33, 4033 (1986).

${ }^{9}$ N. Ramsey, Molecular Beams (Oxford University Press, Oxford, 1956).

${ }^{10}$ F. T. Arrechi, E. Courtnes, R. Gilmore, and H. Thomas, "Atomic coherent states in quantum optics," Phys. Rev. A 6, 6 (1972).

${ }^{11}$ J. Steinbach and C. C. Gerry, "Efficient scheme for the deterministic maximal entanglement of N trapped ions," Phys. Rev. A 81, 5528 (1998).

${ }^{12}$ D. Leibfried, M. D. Barrett, T. Schaetz, J. Briton, J. Chiaverini, W. M. Itano, J. D. Jost, C. Langer, and D. J. Wineland, "Toward Heisenberglimited spectroscopy with multiparticle entangled states," Science 304, 1476 (2004).

${ }^{13}$ D. T. Pegg and S. M. Barnett, "Phase properties of the quantized singlemode electromagnetic field," Phys. Rev. A 39, 1665 (1989).

${ }^{14}$ L. Pezzé and A. Smerzi, Quantum theory of phase estimation (Atom Interferometry: Proceedings of the International School of Physics Enrico Fermi, edited by G. M. Tino and M. A. Kasevich, IOS, Amsterdam, p.691, 2014).

${ }^{15}$ The derivation of the CRB is straightforward. First, we have $\frac{\partial\langle\Phi\rangle_{\varphi}}{\partial \varphi}=$ $\sum_{\varepsilon} \partial_{\varphi} P(\varepsilon \mid \varphi) \Phi(\varepsilon)=\left\langle\Phi \frac{\partial L}{\partial \varphi}\right\rangle$, where $L(\varepsilon \mid \varphi) \equiv \ln P(\varepsilon \mid \varphi)$. Noting that $\sum_{\varepsilon} \partial_{\varphi} P(\varepsilon \mid \varphi)=\left\langle\frac{\partial L}{\partial \varphi}\right\rangle=0$ we have $\left(\frac{\partial\langle\Phi\rangle_{\varphi}}{\partial \varphi}\right)^{2}=\left\langle\left(\Phi-\langle\Phi\rangle_{\varphi}\right) \frac{\partial L}{\partial \varphi}\right\rangle_{\varphi}^{2} \leq$ $\left\langle\left(\Phi-\langle\Phi\rangle_{\varphi}\right)^{2}\right\rangle_{\varphi}\left\langle\left(\frac{\partial L}{\partial \varphi}\right)^{2}\right\rangle_{\varphi}=(\Delta \Phi)_{\varphi}^{2} F(\varphi)$, where we have invoked the Cauchy-Schwarz inequality $\langle A B\rangle_{\varphi}^{2} \leq\left\langle A^{2}\right\rangle_{\varphi}\left\langle B^{2}\right\rangle_{\varphi}$. Dividing by $F(\varphi)$ yields the CRB in $\mathrm{Eq} 28$

${ }^{16} \mathrm{We}$ follow the language found in the literature with regards to defining the upper and lower bounds on phase estimation; i.e. the CRB is the upper bound and the qCRB is the lower bound.

${ }^{17}$ S. L. Braunstein and C. M. Caves, "Statistical distance and the geometry of quantum states," Phys. Rev. Lett. 72, 3439 (1994).

${ }^{18}$ S. L. Braunstein, G. J. Milburn, and C. M. Caves, "Generalized uncertainty relations: theory, examples and Lorentz invariants," Ann. of Phys. 247 (1996).

${ }^{19}$ The QFI in Eq. 31 has a pleasing geometrical interpretation: it is the infinitesimal version of the quantum fidelity $\mathscr{F}_{Q}\left(\hat{\rho}_{1}, \hat{\rho}_{2}\right)=$ $\operatorname{Tr}\left[\sqrt{\sqrt{\hat{\rho}_{2}} \hat{\rho}_{1} \sqrt{\hat{\rho}_{2}}}\right]$ between two density matrices in the sense that $\int_{0}^{\zeta} \sqrt{F_{Q}}\left(\zeta^{\prime}\right) d \zeta^{\prime}=\sqrt{\mathscr{F}_{Q}}(\zeta)$ along the geodesic curve connecting $\hat{\rho}_{1}$ and $\hat{\rho}_{2}$ parameterized by $\zeta$. This can be seen a follows: $\mathscr{F}_{Q}\left(\hat{\rho}_{1}, \hat{\rho}_{2}\right)=$ $\max _{U} \operatorname{Tr}\left[\sqrt{\hat{\rho}_{1}} \sqrt{\hat{\rho}_{2}}\right]=\operatorname{Tr}\left[\left|\sqrt{\hat{\rho}_{1}} \sqrt{\hat{\rho}_{2}}\right|\right]=\left(\right.$ where $\left.|A|=\sqrt{A A^{\dagger}}\right)$ over all purifications of the density matrices. A purification of a density matrix $\hat{\rho}_{i}$ is a pure state $\left|\psi_{i}\right\rangle=\left(U^{(f)} \otimes \sqrt{\hat{\rho}_{i}}\right)|\Gamma\rangle$ where $|\Gamma\rangle=\sum_{k}|k\rangle \otimes|k|^{\sqrt{97} \text { such }}$ that $\operatorname{Tr}_{f}\left[\left|\psi_{i}\right\rangle\left\langle\psi_{i}\right|\right]=\hat{\rho}_{i}$. We can think of this as a fibre bundle where the base space is the space of all positive Hermitian operators, not necessarily of unit trace (the positive cone), and sitting above each (unnormalized) quantum state $\hat{\rho}_{i}$ is the vector space (fibre, $f$ ) of its purifications, which as operators can be represented the vector $A_{i}=\sqrt{\hat{\rho}_{i}}$. The arbitrary unitary $U^{(f)}$ is the freedom to move the vector $A_{i}$ around in the fibre. Now the fidelity is given as $\sqrt{\mathscr{F}_{Q}}(\zeta)=\max \left|\operatorname{Tr}\left[A_{1} A_{2}^{\dagger}\right]\right|$ over all purifications (i.e. over all $\left.U_{1}^{(f)} U_{2}^{\dagger(f)}\right)$. The Bures angle $d_{B}$ is given by $\cos \left[d_{B}\left(\hat{\rho}_{1}, \hat{\rho}_{2}\right)\right]=\sqrt{\mathscr{F}_{Q}}\left(\hat{\rho}_{1}, \hat{\rho}_{2}\right)$ is the length of the geodesic curve within the subspace of (unit trace) density matrices connecting $\hat{\rho}_{1}$ and $\hat{\rho}_{2}$. The infinitesimal version of this is given by the Bures metric ${ }^{96} d s_{B}^{2}=$ $\operatorname{Tr}\left[d A_{1} d A_{2}^{\dagger}\right]=\frac{1}{4} \operatorname{Tr}\left[d \hat{\rho}(\zeta) L_{\zeta}\right]=\frac{1}{4} \operatorname{Tr}\left[\hat{\rho}(\zeta) L_{\zeta}^{2}\right]=F_{Q}(\zeta) d \zeta^{2}$, where the last expression is just Eq. 31 This last expression could also be interpreted as the speed $d s_{B} / d \zeta=\sqrt{F_{Q}}(\zeta)$ along the geodesic connecting the two quantum states $\hat{\rho}_{1}$ and $\hat{\rho}_{2}$ (our input and output states along which $\zeta$ varies) is governed by the (square root) of the QFI. Note further that for pure states $\frac{1}{4} F_{Q}(\zeta)=\left[\left\langle\partial_{\zeta} \psi \mid \partial_{\zeta} \psi\right\rangle-\left|\left\langle\psi \mid \partial_{\zeta} \psi\right\rangle\right|^{2}\right]=\operatorname{Tr}\left[\left|\partial_{\zeta} \psi\right\rangle\left\langle\partial_{\zeta} \psi\right| P_{\perp}\right]$ where $P_{\perp}=I-|\psi\rangle\langle\psi|$ is the projector onto states perpendicular to $|\psi\rangle$. Thus $\left|\nabla_{\zeta} \psi\right\rangle \equiv P_{\perp}\left|\partial_{\zeta} \psi\right\rangle=\left|\partial_{\zeta} \psi\right\rangle-|\psi\rangle\left\langle\psi \mid \partial_{\zeta} \psi\right\rangle$ is the intrinsic covariant derivative $\sqrt{101}$ pointing across fibres that is horizontal (tangent) to the base (parameter, $\zeta$ ) space, with $\left\langle\psi \mid \partial_{\zeta} \psi\right\rangle$ the $\mathrm{U}(1)$ connection (in the complex Hermitian line bundle $\sqrt{96102103}$. The QFI is just the norm of this covariant derivative, $\frac{1}{4} F_{Q}(\zeta)=\|\left|\nabla_{\zeta} \psi\right\rangle \|^{2}=\left\langle\nabla_{\zeta} \psi \mid \nabla_{\zeta} \psi\right\rangle$. From the discussion after Eq. 54 with the parity operator considered as a unitary evolution operator $|\psi(\phi)\rangle=\hat{\Pi}_{b}^{(\phi)}|\psi(0)\rangle=e^{-i \phi \hat{n}_{b}}|\psi(0)\rangle$ we obtain $\left|\nabla_{\phi} \psi\right\rangle=-i\left(\hat{n}_{b}-\right.$ $\left.\bar{n}_{b}\right)|\psi(\phi)\rangle$, with $\bar{n}_{b}=\left\langle\psi(\phi)\left|\hat{n}_{b}\right| \psi(\phi)\right\rangle$, and $F_{\Pi_{b}}(\phi)=4 \|\left|\nabla_{\phi} \psi\right\rangle \|^{2}=$ $4\left\langle\left(\Delta \hat{n}_{b}\right)^{2}\right\rangle_{\phi}$ as before. Note, these geometric concepts can be extended to multiparameter estimatior ${ }^{98}$, where $H=H\left(\zeta_{1}, \zeta_{2}, \ldots\right)$ and the Quantum Geometric Tensor $Q_{i j}=\left\langle\partial_{i} \psi\left|P_{\perp}\right| \partial_{i} \psi\right\rangle=\left\langle\partial_{i} H \partial_{j} H\right\rangle-\left\langle\partial_{i} H\right\rangle\left\langle\partial_{j} H\right\rangle$ (where $\left.\partial_{i}=\partial_{\zeta_{i}}\right)$ takes central role 17 , with the unifying properties that $\operatorname{Re}\left(Q_{i j}\right)=$ $\frac{1}{4} \mathscr{F}_{Q F I}=\operatorname{Cov}\left(H_{i}, H_{j}\right)=\left\langle\frac{1}{2}\left\{H_{i} H_{j}\right\}\right\rangle-\left\langle H_{i}\right\rangle\left\langle H_{j}\right\rangle$ are the elements of the QFI Matrix ${ }^{177}$, and $\operatorname{Im}\left(Q_{i j}\right)=-\frac{1}{2} \Omega_{i j}=-\frac{1}{4}\left(\left\langle\partial_{i} \psi \mid \partial_{j} \psi\right\rangle-\left\langle\partial_{j} \psi \mid \partial_{i} \psi\right\rangle\right)$ are the Berry (Phase) Curvatures 99100102 .

${ }^{20}$ P. D. Drummond and M. Hillery, "The quantum theory of nonlinear optics," Acta Physica Slovaca 59 (2009).

${ }^{21}$ C. W. Helstrom, "Minimum mean-squared error of estimates in quantum statistics," Phys. Lett. A 25, 101-102 (1967).

${ }^{22}$ M. Hubner, "Explicit computation of the Bures distance for density matricies," Phys. Lett. A 163, 239-242 (1992).

${ }^{23}$ C. C. Gerry, "Heisenberg-limit interferometry with four-wave mixers operating in a nonlinear regime," Phys. Rev. A 61, 043811 (2000).

${ }^{24} \mathrm{C}$. Gerry and J. Mimih, "The parity operator in quantum optical metrology," Contemp. Phys. 51, 6 (2010).

${ }^{25}$ H. F. Hofmann, "All path-symmetric pure states achieve their maximal phase sensitivity in conventional two-path interferometry," Phys. Lett. A 79, 033822 (2009).

${ }^{26}$ S. Kim, K. P. Seshadreesan, J. P. Dowling, and H. Lee, "Parity measurement is sufficient for phase estimation at the quantum cramér-rao bound for path symmetric states," ArXiv: 1207.049 (2012).

${ }^{27}$ K. P. Seshadreesan, S. Kim, J. P. Dowling, and H. Lee, "Phase estimation and the quantum Cramér-Rao bound via parity detection," Phys. Lett. A 87, 043833 (2013).

${ }^{28} \mathrm{H}$. Uys and P. Meystre, "Quantum states for Heisenberg-limited interferometry," Phys. Lett. A 76, 013804 (2007).

${ }^{29}$ The exception being the optical $N 00 N$ state, for which parity is optimal for all values of the phase.

${ }^{30} \mathrm{O}$. E. Barndorff-Nielsen and R. D. Gill, "Fisher information in quantum statistics," J. Phys. A 33, 24 (2000).

${ }^{31}$ P. M. Anisimov, G. M. Raterman, A. Chiruvelli, W. N. Plick, and S. D. Huver, "Quantum metrology with two-mode squeezed vacuum: parity detection beats the Heisenberg limit," Phys. Rev. Lett. 104, 103602 (2010).

${ }^{32}$ Generally speaking, the expectation value of the parity operator for each output mode is related by a phase shift. Often the output mode for which the parity expectation value peaks at $\phi=0$ is considered. However, either output mode is suitable with the right choice of phase-shifter.

${ }^{33}$ J. P. Dowling, "Correlated input-port matter-wave interferometer: quantum-noise limits to the atom-laser gyroscope,” Phys. Rev. A 57, 4736 
(1998).

${ }^{34}$ H. Lee, P. Kok, and J. P. Dowling, "A quantum Rosetta stone for interferometry,” J. Mod. Opt. 49, 2325 (2002).

${ }^{35}$ P. Kok, S. L. Braunstein, and J. P. Dowling, "Quantum lithography, entanglement and Heisenberg-limited parameter estimation," J. Opt. B 6, 8 (2004).

${ }^{36}$ J. Joo, W. J. Munro, and T. P. Spiller, "Quantum metrology with entangled coherent states," Phys. Rev. Lett. 107, 083601 (2011).

${ }^{37}$ Y. Israel, L. Cohen, X. Song, J. Joo, and H. S. Eisenberg, "Entangled coherent states created by mixing squeezed vacuum and coherent light," Optica 6, 6 (2019).

${ }^{38}$ B. Yurke and D. Stoler, "Generating quantum mechanical superpositions of macroscopically distinguishable states via amplitude dispersion," Phys. Rev. Lett. 57, 13 (1986).

${ }^{39}$ A. Luis, "Equivalence between macroscopic quantum superpositions and maxmially entangled states: application to phase-shift detection," Phys. Rev. A 64, 054102 (2001).

${ }^{40}$ C. C. Gerry, A. Benmoussa, and R. A. Campos, "Nonlinear interferometer as a resource for maximally entangled photonic states: application to interferometry," Phys. Rev. A 66, 013804 (2002).

${ }^{41}$ M. J. Holland and K. Burnett, "Interferometric detection of optical phase shifts at the Heisenberg limit," Phys. Rev. Lett. 71, 1355 (1993).

${ }^{42}$ Their discussion is in the context of spectroscopy using maximally entangled states of a system of $N$ two-level trapped ions.

${ }^{43}$ C. C. Gerry and R. A. Campos, "Generation of maximally entangled photonic states with a quantum-optical Fredkin gate," Phys. Rev. A 64, 063814 (2001).

${ }^{44}$ C. C. Gerry and A. Benmoussa, "Heisenberg-limited interferometry and photolithograpy with nonlinear four-wave mixing," Phys. Rev. A 65, 033822 (2001).

${ }^{45}$ P. Kok, H. Lee, and J. P. Dowling, "Creation of large-photon-number path entanglement conditioned on photodetection," Phys. Rev. A 65, 052104 (2002).

${ }^{46}$ H. Lee, P. Kok, and N. J. C. amd J. P. Dowling, "Linear optics and projective measurements alone suffice to create large-photon-number path entanglement," Phys. Rev. A 65, 030101 (2002).

${ }^{47}$ J. Fiurášek, "Conditional generation of N-photon entangled states of light," Phys. Rev. A 65, 053818 (2002).

${ }^{48}$ C. C. Gerry and A. Benmoussa, "Nonlinear interferometer as a resource for maximally entangled photonic states: application to interferometry," Phys. Rev. A 66, 013804 (2002).

${ }^{49}$ R. A. Campos, C. C. Gerry, and A. Benmoussa, "Optical interferometry at the Heisenberg limit with twin Fock states and parity measurements," Phys. Rev. A 68, 023810 (2003).

${ }^{50}$ C. K. Hong, Z. Y. Ou, and L. Mandel, "Measurement of sub-picosecond time interverals between two photons by interference," Phys. Rev. Lett. 59, 2044 (1987).

${ }^{51}$ W. Feller, An Introduction to Probability Theory and its Applications, 3rd Edition (Wiley, 1968).

${ }^{52}$ R. W. Boyd, Nonlinear Optics (Academic Press, 1992).

${ }^{53} \mathrm{C}$. C. Gerry and J. Mimih, "Heisenberg-limited interferometry with pair coherent states and parity measurements," Phys. Rev. A 82, 013831 (2010).

${ }^{54}$ R. Birrittella, A. Gura, and C. C. Gerry, "Coherently stimulated parametric down-conversion, phase effects, and quantum optical interferometry," Phys. Rev. A 91, 053801 (2015).

${ }^{55}$ R. Birrittella, P. M. Alsing, and C. C. Gerry, "Phase effects in coherently stimulated down conversion with a quantized pump field," Phys. Rev. A 101, 013813 (2019).

${ }^{56} \mathrm{G}$. S. Agarwal, "Generation of pair coherent states and squeezing via the competition of four-wave mixing and amplified spontaneous emission," Phys. Rev. Lett. 57, 827 (1986).

${ }^{57}$ The generalized pair coherent state is defined such that $\left(\hat{a}^{\dagger} \hat{a}-\hat{b}^{\dagger} \hat{b}\right)|\zeta, q\rangle=q|\zeta, q\rangle$. For the purposes of this review article, we assume $q=0$ without loss of generality.

${ }^{58}$ C. C. Gerry, J. Mimih, and R. Birrittella, "State-projective scheme for generating pair coherent states in traveling-wave optical fields," Phys. Rev. A 84, 023810 (2011).

${ }^{59}$ C. M. Caves, "Quantum-mechanical noise in an interferometer," Phys. Rev. D 23, 9 (1981).

${ }^{60} \mathrm{~V}$. Buzěk and M. Hillery, "Quantum disentanglement and phase measure- ment," Czech. J. Phys. B 45, 711-726 (1995).

${ }^{61}$ L. Pezzé and A. Smerzi, "Mach-Zehnder interferometry at the Heisenberg limit with coherent and squeezed-vacuum light," Phys. Rev. Lett. 100, 073601 (2008).

${ }^{62}$ R. Birrittella, J. Mimih, and C. C. Gerry, "Multiphoton quantum interference at a beam splitter and the approach to Heisenberg-limited interferometry," Phys. Rev. A 86, 063828 (2012)

${ }^{63}$ I. Afek, O. Ambar, and Y. Silberberg, "High N00N states by mixing quantum and classical light," Science 328, (5980) 879-881 (2010).

${ }^{64}$ K. P. Seshadreesan, P. M. Anisimov, H. Lee, and J. P. Dowling, "Parity detection achieves the Heisenberg limit in interferometry with coherent mixed with squeezed vacuum light," New J. Phys. 13, 083026 (2011).

${ }^{65} \mathrm{R}$. Birrittella and C. C. Gerry, "Quantum optical interferometry via the mixing of coherent and photon-subtracted squeezed vacuum states of light,” J. Opt. Soc. Am. B 31, 3 (2014).

${ }^{66} \mathrm{~A}$. Chiruvelli and H. Lee, "Parity measurements in quantum optical metrology," J. Mod. Opt. 58, 945-953 (2011).

${ }^{67}$ Y. Gao, P. Anisimov, C. F. Wildfeuer, J. Luine, H. Lee, and J. P. Dowling, "Super-resolution at the shot-noise limit with coherent states and photonnumber-resolving detectors," J. Opt. Soc. Am. B 27, 6 (2010).

${ }^{68}$ C. C. Gerry and P. L. Knight, Introductory Quantum Optics (Cambridge University Press, Cambridge, UK., 2005).

${ }^{69}$ K. J. Resch, K. L. Pregnell, R. Prevedel, A. Gilchrist, G. J. Pryde, J. L. O'Brien, and A. G. White, "Time-reversal and super-resolving phase measurements," Phys. Rev. Lett. 98, 223601 (2007).

${ }^{70}$ D. Li, B. T. Gard, Y. Gao, C. Yuan, W. Zhang, H. Lee, and J. P. Dowling, "Phase sensitivity at the Heisenberg limit in an SU(1,1) interferometer via parity detection,” Phys. Rev. A 94, 063840 (2016).

${ }^{71}$ D. B. Hume, I. Stroescu, M. Joos, W. Muessel, H. Strobel, and M. K. Oberthaler, "Accurate atom counting in mesoscopic ensembles," Phys. Rev. Lett. 111, 253001 (2013).

${ }^{72}$ C. C. Gerry, A. Benmoussa, and R. A. Campos, "Quantum nondemolition measurement of parity and generation of parity eigenstates in optical fields," Phys. Rev. A 72, 053818 (2005).

${ }^{73}$ L. Sun, A. Petrenko, Z. Leghtas, B. Vlastakis, G. Kirchmair, K. M. Sliwa, A. Narla, M. Hatridge, S. Shankar, J. Blumoff, L. Frunzio, M. Mirrahimi, M. H. Devoret, and R. J. Schoelkopf, "Tracking photon jumps with repeated quantum non-demolition parity measurements," Nature 511, 444448 (2014).

${ }^{74}$ S. Rosenblum, P. Reinhold, M. Mirrahimi, L. Jiang, L. Frunzio, and R. J. Schoelkopf, "Fault-tolerant detection of a quantum error," Science 361, 6399 pp. 266-270 (2018).

${ }^{75} \mathrm{H}$. K. Lao and M. B. Plenio, "Universal quantum computing with arbitrary continuous-variable encoding," Phys. Rev. Lett. 117, 100501 (2016).

${ }^{76}$ Y. Y. Gao, B. J. Lester, K. S. Chou, L. Frunzio, M. H. Devoret, L. Jiang, S. M. Girvin, and R. J. Schoelkopf, "Entanglement of bosonic modes through an engineered exchange interaction," Nature 566, 509-512 (2019).

${ }^{77}$ L. Cohen, D. Istrati, L. Dovratm, and H. S. Eisenberg, "Super-resolved phase measurements at the shot noise limit by parity measurements," Optics Express 22, 11945 (2014).

${ }^{78} \mathrm{P}$. Kok and B. W. Lovett, Introduction to Optical Quantum Information Processing (Cambridge University Press, Cambridge, UK., p. 124, 2010).

${ }^{79}$ C. Silberhorn, "Detecting quantum light," Contemp. Phys. 48, 143 (2007).

${ }^{80}$ T. Gerrits, S. Clancy, T. S. Clement, B. Calkins, A. E. Lita, A. J. Miller, A. L. Migdall, S. W. Nam, R. P. Mirin, and E. Knill, "Generation of optical coherent-state superpositions by number-resolving photon subtraction from the squeezed vacuum," Phys. Rev. A 82, 031802(R) (2010).

${ }^{81}$ K. Banaszek and I. A. Walmsley, "Photon counting with a loop detector," Opt. Lett. 28, 52 (2003).

${ }^{82}$ D. Achilles, C. Silberhorn, C. Śliwa, K. Banaszek, I. A. Walmsley, M. J. Finch, B. C. Jacobs, T. B. Pittman, and J. D. Franson, "Photon-number resolving detection using time-multiplexing," J. Mod. Opt. 51, 1499 (2004).

${ }^{83}$ L. A. Jiang, E. A. Dauler, and J. T. Chang, "Photon-number-resolving detector with 10 bits of resolution," Phys. Rev. A 75, 062325 (2007).

${ }^{84}$ L. Cohen, Y. Pilnyak, D. Istrali, N. M. Studer, J. P. Dowling, and H. Eisenberg, "Absolute calibration of single-photon and multiplexed photonnumber-resolving detectors," Phys. Rev. A 98, 013811 (2018).

${ }^{85} \mathrm{~N}$. Imoto, H. A. Haus, and Y. Yamamoto, "Quantum nondemolition measurements of the photon number via the optical Kerr effect," Phys. Rev. A 32, 2287 (1985). 
${ }^{86}$ W. N. Plick, P. M. Anisimov, J. P. Dowling, H. Lee, and G. S. Agarwal, "Parity detection in quantum optical metrology without number resolving detectors," N. J. Phys. 12, 113025 (2010).

${ }^{87}$ M. Herrero-Collantes and J. C. Garcia-Escartin, "Quantum random number generators," Rev. Mod. Phys. 89, 015004 (2017).

${ }^{88}$ J. Huang, S. Wu, H. Zhong, and C. Lee, Annual Review of Cold Atoms anf Molecules, Chapter 7: Quantum Metrology with Cold Atoms, pp. 365-415 (World Scientific, 2014).

${ }^{89}$ L. Pezzé, A. Smerzi, M. K. Overthaler, R. Schmied, and P. Treutlein, "Quantum metrology with nonclassical states of atomic ensembles," Rev. Mod. Phys. 90, 035005 (2018).

${ }^{90}$ For complex state coefficients,Eq. 142 can be written as $\left\langle\hat{J}_{z}\right\rangle=$ $2 \operatorname{Re}\left[z e^{i \phi}\right]+\alpha^{(j)}$, where $z$ is a complex number expressed in terms of the state coefficients. For real state coefficients, this simplifies to the form of Eq. 143

${ }^{91}$ R. H. Dicke, "Coherent in spontaneous radiation processes," Phys. Rev. 93, 99 (1954)

${ }^{92}$ J. M. Radcliffe, "Some properties of coherent spin states," J. Phys. A: Gen. Phys. 4, 313 (1971).

${ }^{93}$ M. E. Rose, Elementary Theory of Angular Momentum (Dover Publications, Inc., New York, 1995).
${ }^{94}$ Note that $\hat{D}_{a}(\alpha) \hat{D}_{a}(\beta)=e^{\frac{1}{2}\left(\alpha \beta^{*}-\alpha^{*} \beta\right)} \hat{D}_{a}(\alpha+\beta)$. The product of displacement operators is the sum of the displacements up to an overall phase factor.

${ }^{95} \mathrm{~N}$. Tajima, "Analytical formula for numerical evaluations of the Wigner rotation matricies at high spins," Phys. Rev. C 91, 014320 (2015).

${ }^{96}$ I. Bengtsson and K. Zyczkowski, Geometry of Quantum States (Cambridge University Press, Cambridge, UK., 2006).

${ }^{97} \mathrm{M}$. Wilde, Quantum Information, 2nd Ed. (Cambridge University Press, Cambridge, UK., 2017).

${ }^{98}$ X. J. L. F. W. Guo, W. Zhong and X. Wang, "Berry curvature as a lower bound for multiparameter estimation," Phys. Rev. A 93, 042115 (2019).

${ }^{99}$ J. Samuel and R. Bhandari, "General setting for berry's phase," Phys. Rev. Lett. 60, 2339 (1988).

${ }^{100}$ A. Shapere and F. Wilczek, Geometric Phases in Physics (World Scientific, Singapore, 1989) pp. 113-123.

${ }^{101} \mathrm{~J}$. Provost and G. Vallee, "Riemann structure on manifolds of quantum states," Comm. Math. Phys. 76, 289 (1980).

${ }^{102}$ Y. Ben-Aryeh, "Berry and Pancharatnam topological phases of atomic and optical systems," J. Opt. B: Quantum Semiclass. Opt. 6, R1-R18 (2004).

${ }^{103}$ T. Frankel, The Geometry of Physics, 3rd Ed., Chap. 17 (Cambridge University Press, Cambridge, UK., 2012) pp. 451-474. 